Supporting Information

\title{
A Square-Planar Cobalt(III) Pincer Complex
}

Paraskevi O. Lagaditis, Bastian Schluschaß, Serhiy Demeshko, Christian Würtele, and Sven Schneider*

Institut für Anorganische Chemie, Georg-August-Universität, Tammannstraße 4, 37077 Göttingen, Germany. 


\section{Cyclic Voltammetry}

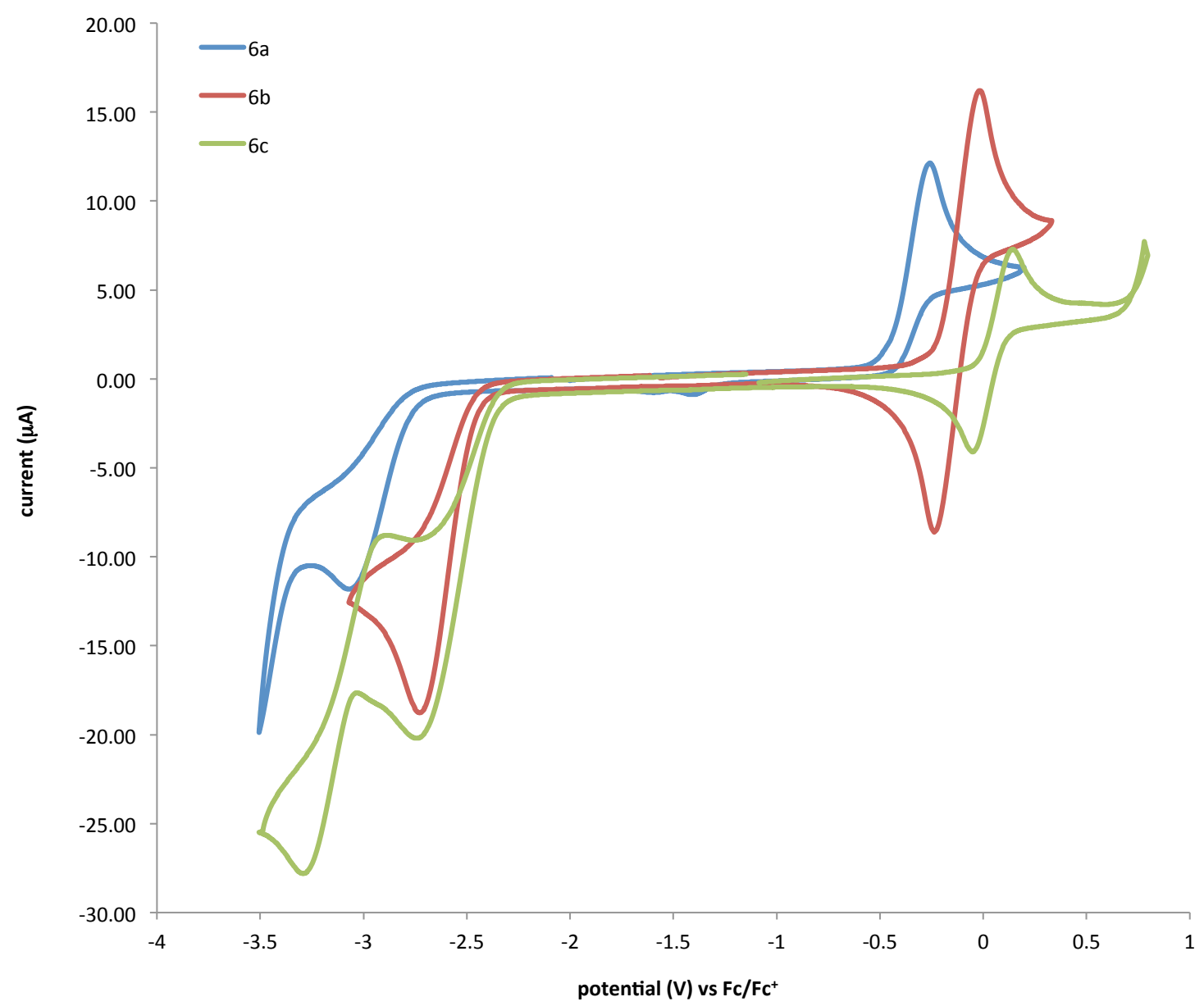

Figure S1. Cyclic voltammograms of $\mathbf{6 a - c}$ in $\mathrm{THF}\left(0.1 \mathrm{M}\left[n \mathrm{Bu}_{4} \mathrm{~N}\right]\left[\mathrm{PF}_{6}\right]\right.$, r.t., glassy carbon working electrode, scan rate $100 \mathrm{mV} / \mathrm{s})$. 


\section{Crystallographic Details}

2.1 X-ray Single-Crystal Structure Analysis of 5.

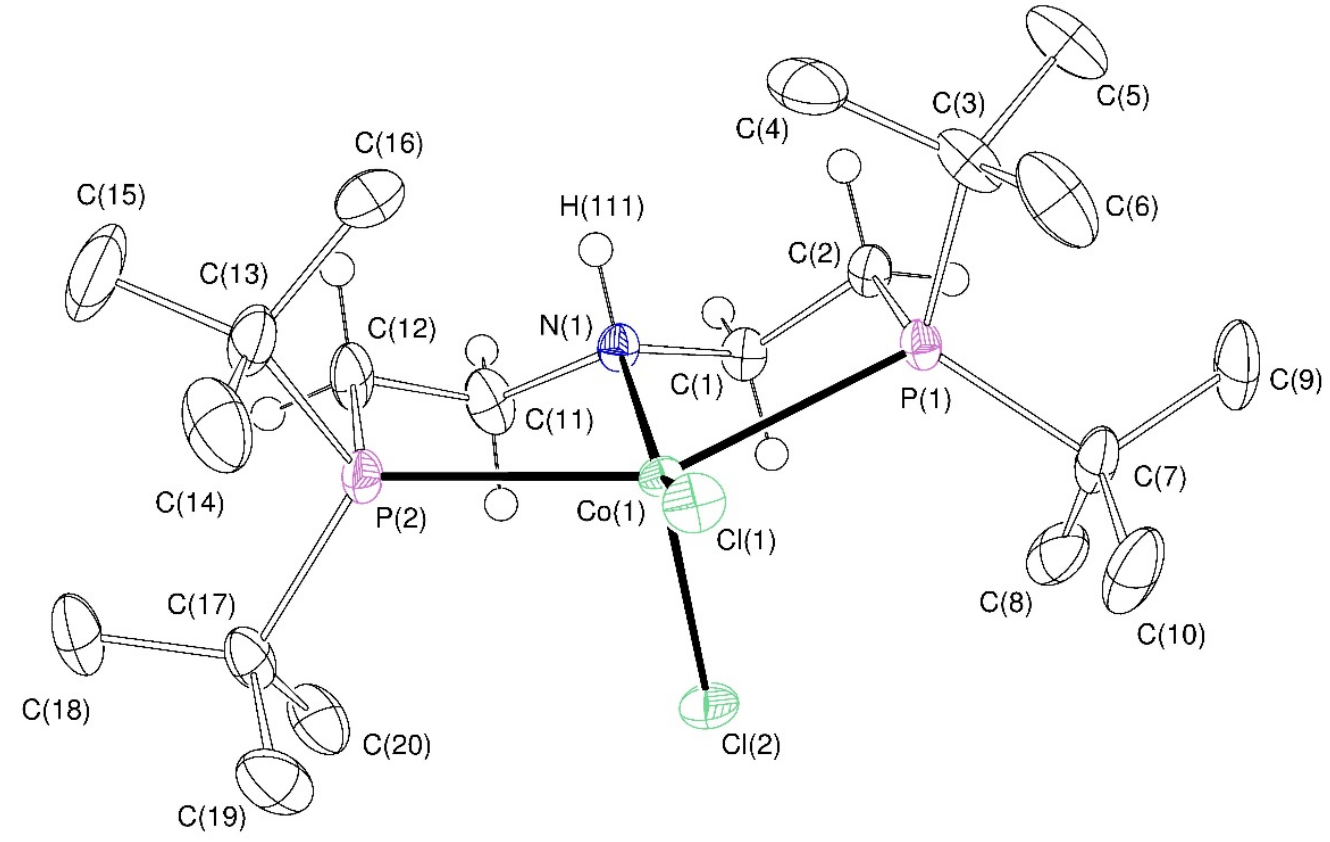

Figure S2. Thermal ellipsoid plot of $\mathbf{5}$ with the anisotropic displacement parameters drawn at the $50 \%$ probability level. The N-H hydrogen atom was found and isotropically refined. All hydrogen atoms of the $t \mathrm{Bu}-$ groups are omitted for clarity.

Table S1. Crystal data and structure refinement for 5 .

CCDC-No.

Diffractometer and Detector

Identification code

Empirical formula

Formula weight

Temperature

Wavelength

Crystal system

Space group

Unit cell dimensions

Volume

Z

Density (calculated)

Absorption coefficient
1452220

\section{Bruker, CMOS}

mo_TEST_050115_0m_a

$\mathrm{C}_{20} \mathrm{H}_{45} \mathrm{Cl}_{2} \mathrm{CoNP}_{2}$

491.34

173(2) K

$0.71073 \AA$

Monoclinic

$\mathrm{P} 2{ }_{1}$

$$
\begin{array}{ll}
\mathrm{a}=7.0747(2) \AA & \alpha=90^{\circ} \\
\mathrm{b}=14.7836(5) \AA & \beta=91.112(2)^{\circ} \\
\mathrm{c}=12.4276(4) \AA & \gamma=90^{\circ}
\end{array}
$$$$
1299.55(7) \AA^{3}
$$

2

$1.256 \mathrm{Mg} / \mathrm{m}^{3}$

$0.995 \mathrm{~mm}^{-1}$ 
$\mathrm{F}(000)$

Crystal shape and color:

Crystal size

Theta range for data collection

Index ranges

Reflections collected

Independent reflections

Completeness to theta $=25.242^{\circ}$

Absorption correction

Max. and min. transmission

Refinement method

Data / restraints / parameters

Goodness-of-fit on $\mathrm{F}^{2}$

Final R indices [I $>2 \operatorname{sigma}(\mathrm{I})]$

$\mathrm{R}$ indices (all data)

Absolute structure parameter

Largest diff. peak and hole
526

Block, blue

$0.275 \times 0.236 \times 0.124 \mathrm{~mm}^{3}$

2.141 to $30.577^{\circ}$

$-10<=\mathrm{h}<=10,-21<=\mathrm{k}<=21,-17<=\mathrm{k}<=17$

42929

$7947[\mathrm{R}(\mathrm{int})=0.0472]$

$100.0 \%$

Semi-empirical from equivalents

0.7461 and 0.6787

Full-matrix least-squares on $\mathrm{F}^{2}$

7947 / 1 / 251

1.020

$\mathrm{R} 1=0.0282, \mathrm{wR} 2=0.0483$

$\mathrm{R} 1=0.0402, \mathrm{wR} 2=0.0510$

$0.003(5)$

0.264 and -0.379 e $\AA^{-3}$

Table S2. Bond lengths $[\AA]$ and angles $\left[{ }^{\circ}\right]$ for 5.

$\begin{array}{llll}\mathrm{Co}(1)-\mathrm{N}(1) & 2.1772(16) & \mathrm{C}(7)-\mathrm{P}(1)-\mathrm{Co}(1) & 121.35(8) \\ \mathrm{Co}(1)-\mathrm{Cl}(2) & 2.2873(6) & \mathrm{C}(3)-\mathrm{P}(1)-\mathrm{Co}(1) & 115.27(7) \\ \mathrm{Co}(1)-\mathrm{Cl}(1) & 2.3264(5) & \mathrm{C}(11)-\mathrm{N}(1)-\mathrm{C}(1) & 110.22(17) \\ \mathrm{Co}(1)-\mathrm{P}(1) & 2.5761(6) & \mathrm{C}(11)-\mathrm{N}(1)-\mathrm{Co}(1) & 111.39(12) \\ \mathrm{Co}(1)-\mathrm{P}(2) & 2.5789(6) & \mathrm{C}(1)-\mathrm{N}(1)-\mathrm{Co}(1) & 110.71(12) \\ \mathrm{P}(1)-\mathrm{C}(2) & 1.841(2) & \mathrm{C}(11)-\mathrm{N}(1)-\mathrm{H}(111) & 102.5(15) \\ \mathrm{P}(1)-\mathrm{C}(7) & 1.883(2) & \mathrm{C}(1)-\mathrm{N}(1)-\mathrm{H}(111) & 108.0(14) \\ \mathrm{P}(1)-\mathrm{C}(3) & 1.885(2) & \mathrm{Co}(1)-\mathrm{N}(1)-\mathrm{H}(111) & 113.7(15) \\ \mathrm{N}(1)-\mathrm{C}(11) & 1.482(3) & \mathrm{N}(1)-\mathrm{C}(1)-\mathrm{C}(2) & 108.24(16) \\ \mathrm{N}(1)-\mathrm{C}(1) & 1.482(3) & \mathrm{C}(12)-\mathrm{P}(2)-\mathrm{C}(13) & 103.53(12) \\ \mathrm{N}(1)-\mathrm{H}(111) & 0.92(2) & \mathrm{C}(12)-\mathrm{P}(2)-\mathrm{C}(17) & 102.84(11) \\ \mathrm{C}(1)-\mathrm{C}(2) & 1.521(3) & \mathrm{C}(13)-\mathrm{P}(2)-\mathrm{C}(17) & 111.70(11) \\ \mathrm{P}(2)-\mathrm{C}(12) & 1.839(2) & \mathrm{C}(12)-\mathrm{P}(2)-\mathrm{Co}(1) & 97.44(7) \\ \mathrm{P}(2)-\mathrm{C}(13) & 1.881(3) & \mathrm{C}(13)-\mathrm{P}(2)-\mathrm{Co}(1) & 115.30(8) \\ \mathrm{P}(2)-\mathrm{C}(17) & 1.889(2) & \mathrm{C}(17)-\mathrm{P}(2)-\mathrm{Co}(1) & 121.95(8) \\ \mathrm{C}(3)-\mathrm{C}(4) & 1.524(4) & \mathrm{C}(1)-\mathrm{C}(2)-\mathrm{P}(1) & 109.80(14) \\ \mathrm{C}(3)-\mathrm{C}(6) & 1.536(3) & \mathrm{C}(4)-\mathrm{C}(3)-\mathrm{C}(6) & 108.3(2) \\ \mathrm{C}(3)-\mathrm{C}(5) & 1.542(3) & \mathrm{C}(4)-\mathrm{C}(3)-\mathrm{C}(5) & 108.5(2) \\ \mathrm{C}(7)-\mathrm{C}(8) & 1.529(4) & \mathrm{C}(6)-\mathrm{C}(3)-\mathrm{C}(5) & 109.9(2) \\ \mathrm{C}(7)-\mathrm{C}(9) & 1.531(3) & \mathrm{C}(4)-\mathrm{C}(3)-\mathrm{P}(1) & \\ & & & \\ & & & \end{array}$




$\begin{array}{lcll}\mathrm{C}(7)-\mathrm{C}(10) & 1.532(3) & \mathrm{C}(6)-\mathrm{C}(3)-\mathrm{P}(1) & 109.92(18) \\ \mathrm{C}(11)-\mathrm{C}(12) & 1.524(3) & \mathrm{C}(5)-\mathrm{C}(3)-\mathrm{P}(1) & 115.24(17) \\ \mathrm{C}(13)-\mathrm{C}(14) & 1.527(4) & \mathrm{C}(8)-\mathrm{C}(7)-\mathrm{C}(9) & 108.6(2) \\ \mathrm{C}(13)-\mathrm{C}(15) & 1.536(4) & \mathrm{C}(8)-\mathrm{C}(7)-\mathrm{C}(10) & 108.0(2) \\ \mathrm{C}(13)-\mathrm{C}(16) & 1.537(4) & \mathrm{C}(9)-\mathrm{C}(7)-\mathrm{C}(10) & 110.3(2) \\ \mathrm{C}(17)-\mathrm{C}(19) & 1.524(3) & \mathrm{C}(8)-\mathrm{C}(7)-\mathrm{P}(1) & 108.07(17) \\ \mathrm{C}(17)-\mathrm{C}(18) & 1.529(3) & \mathrm{C}(9)-\mathrm{C}(7)-\mathrm{P}(1) & 114.48(19) \\ \mathrm{C}(17)-\mathrm{C}(20) & 1.529(4) & \mathrm{C}(10)-\mathrm{C}(7)-\mathrm{P}(1) & 107.17(17) \\ \mathrm{N}(1)-\mathrm{Co}(1)-\mathrm{Cl}(2) & 99.98(5) & \mathrm{N}(1)-\mathrm{C}(11)-\mathrm{C}(12) & 108.81(19) \\ \mathrm{N}(1)-\mathrm{Co}(1)-\mathrm{Cl}(1) & 152.07(5) & \mathrm{C}(11)-\mathrm{C}(12)-\mathrm{P}(2) & 110.11(15) \\ \mathrm{Cl}(2)-\mathrm{Co}(1)-\mathrm{Cl}(1) & 107.89(2) & \mathrm{C}(14)-\mathrm{C}(13)-\mathrm{C}(15) & 110.3(2) \\ \mathrm{N}(1)-\mathrm{Co}(1)-\mathrm{P}(1) & 78.15(5) & \mathrm{C}(14)-\mathrm{C}(13)-\mathrm{C}(16) & 107.4(2) \\ \mathrm{Cl}(2)-\mathrm{Co}(1)-\mathrm{P}(1) & 99.82(2) & \mathrm{C}(15)-\mathrm{C}(13)-\mathrm{C}(16) & 108.4(2) \\ \mathrm{Cl}(1)-\mathrm{Co}(1)-\mathrm{P}(1) & 95.053(19) & \mathrm{C}(14)-\mathrm{C}(13)-\mathrm{P}(2) & 110.12(19) \\ \mathrm{N}(1)-\mathrm{Co}(1)-\mathrm{P}(2) & 79.50(5) & \mathrm{C}(15)-\mathrm{C}(13)-\mathrm{P}(2) & 115.2(2) \\ \mathrm{Cl}(2)-\mathrm{Co}(1)-\mathrm{P}(2) & 100.64(2) & \mathrm{C}(16)-\mathrm{C}(13)-\mathrm{P}(2) & 104.87(17) \\ \mathrm{Cl}(1)-\mathrm{Co}(1)-\mathrm{P}(2) & 96.76(2) & \mathrm{C}(19)-\mathrm{C}(17)-\mathrm{C}(18) & 109.8(2) \\ \mathrm{P}(1)-\mathrm{Co}(1)-\mathrm{P}(2) & 151.90(2) & \mathrm{C}(19)-\mathrm{C}(17)-\mathrm{C}(20) & 107.7(2) \\ \mathrm{C}(2)-\mathrm{P}(1)-\mathrm{C}(7) & 103.73(10) & \mathrm{C}(18)-\mathrm{C}(17)-\mathrm{C}(20) & 108.4(2) \\ \mathrm{C}(2)-\mathrm{P}(1)-\mathrm{C}(3) & 102.66(10) & \mathrm{C}(19)-\mathrm{C}(17)-\mathrm{P}(2) & 108.33(16) \\ \mathrm{C}(7)-\mathrm{P}(1)-\mathrm{C}(3) & 111.65(11) & \mathrm{C}(18)-\mathrm{C}(17)-\mathrm{P}(2) & 114.80(18) \\ \mathrm{C}(2)-\mathrm{P}(1)-\mathrm{Co}(1) & 98.37(7) & \mathrm{C}(20)-\mathrm{C}(17)-\mathrm{P}(2) & 107.55(16) \\ & & & \end{array}$

Table S3. Torsion angles $\left[{ }^{\circ}\right]$ for 5 .

$\begin{array}{lccc}\mathrm{C}(11)-\mathrm{N}(1)-\mathrm{C}(1)-\mathrm{C}(2) & 168.98(16) & \mathrm{C}(1)-\mathrm{N}(1)-\mathrm{C}(11)-\mathrm{C}(12) & -172.96(17) \\ \mathrm{Co}(1)-\mathrm{N}(1)-\mathrm{C}(1)-\mathrm{C}(2) & -67.32(17) & \mathrm{Co}(1)-\mathrm{N}(1)-\mathrm{C}(11)-\mathrm{C}(12) & 63.7(2) \\ \mathrm{N}(1)-\mathrm{C}(1)-\mathrm{C}(2)-\mathrm{P}(1) & 56.19(19) & \mathrm{N}(1)-\mathrm{C}(11)-\mathrm{C}(12)-\mathrm{P}(2) & -58.4(2) \\ \mathrm{C}(7)-\mathrm{P}(1)-\mathrm{C}(2)-\mathrm{C}(1) & 103.71(16) & \mathrm{C}(13)-\mathrm{P}(2)-\mathrm{C}(12)-\mathrm{C}(11) & 144.48(17) \\ \mathrm{C}(3)-\mathrm{P}(1)-\mathrm{C}(2)-\mathrm{C}(1) & -139.93(15) & \mathrm{C}(17)-\mathrm{P}(2)-\mathrm{C}(12)-\mathrm{C}(11) & -99.10(18) \\ \mathrm{Co}(1)-\mathrm{P}(1)-\mathrm{C}(2)-\mathrm{C}(1) & -21.55(15) & \mathrm{Co}(1)-\mathrm{P}(2)-\mathrm{C}(12)-\mathrm{C}(11) & 26.14(17) \\ \mathrm{C}(2)-\mathrm{P}(1)-\mathrm{C}(3)-\mathrm{C}(4) & 75.22(18) & \mathrm{C}(12)-\mathrm{P}(2)-\mathrm{C}(13)-\mathrm{C}(14) & 169.85(18) \\ \mathrm{C}(7)-\mathrm{P}(1)-\mathrm{C}(3)-\mathrm{C}(4) & -174.24(16) & \mathrm{C}(17)-\mathrm{P}(2)-\mathrm{C}(13)-\mathrm{C}(14) & 59.9(2) \\ \mathrm{Co}(1)-\mathrm{P}(1)-\mathrm{C}(3)-\mathrm{C}(4) & -30.51(18) & \mathrm{Co}(1)-\mathrm{P}(2)-\mathrm{C}(13)-\mathrm{C}(14) & -85.02(18) \\ \mathrm{C}(2)-\mathrm{P}(1)-\mathrm{C}(3)-\mathrm{C}(6) & -168.64(18) & \mathrm{C}(12)-\mathrm{P}(2)-\mathrm{C}(13)-\mathrm{C}(15) & 44.3(3) \\ \mathrm{C}(7)-\mathrm{P}(1)-\mathrm{C}(3)-\mathrm{C}(6) & -58.1(2) & \mathrm{C}(17)-\mathrm{P}(2)-\mathrm{C}(13)-\mathrm{C}(15) & -65.7(3) \\ \mathrm{Co}(1)-\mathrm{P}(1)-\mathrm{C}(3)-\mathrm{C}(6) & 85.63(18) & \mathrm{Co}(1)-\mathrm{P}(2)-\mathrm{C}(13)-\mathrm{C}(15) & 149.4(2) \\ \mathrm{C}(2)-\mathrm{P}(1)-\mathrm{C}(3)-\mathrm{C}(5) & -43.9(2) & \mathrm{C}(12)-\mathrm{P}(2)-\mathrm{C}(13)-\mathrm{C}(16) & -74.8(2) \\ \mathrm{C}(7)-\mathrm{P}(1)-\mathrm{C}(3)-\mathrm{C}(5) & 66.7(2) & \mathrm{C}(17)-\mathrm{P}(2)-\mathrm{C}(13)-\mathrm{C}(16) & 175.17(17) \\ \mathrm{Co}(1)-\mathrm{P}(1)-\mathrm{C}(3)-\mathrm{C}(5) & -149.59(18) & \mathrm{Co}(1)-\mathrm{P}(2)-\mathrm{C}(13)-\mathrm{C}(16) & 30.3(2) \\ & & \mathrm{S} 6 & \end{array}$




$\begin{array}{lccc}\mathrm{C}(2)-\mathrm{P}(1)-\mathrm{C}(7)-\mathrm{C}(8) & -48.97(18) & \mathrm{C}(12)-\mathrm{P}(2)-\mathrm{C}(17)-\mathrm{C}(19) & 168.24(19) \\ \mathrm{C}(3)-\mathrm{P}(1)-\mathrm{C}(7)-\mathrm{C}(8) & -158.83(16) & \mathrm{C}(13)-\mathrm{P}(2)-\mathrm{C}(17)-\mathrm{C}(19) & -81.3(2) \\ \mathrm{Co}(1)-\mathrm{P}(1)-\mathrm{C}(7)-\mathrm{C}(8) & 59.95(18) & \mathrm{Co}(1)-\mathrm{P}(2)-\mathrm{C}(17)-\mathrm{C}(19) & 60.9(2) \\ \mathrm{C}(2)-\mathrm{P}(1)-\mathrm{C}(7)-\mathrm{C}(9) & 72.2(2) & \mathrm{C}(12)-\mathrm{P}(2)-\mathrm{C}(17)-\mathrm{C}(18) & -68.7(2) \\ \mathrm{C}(3)-\mathrm{P}(1)-\mathrm{C}(7)-\mathrm{C}(9) & -37.7(2) & \mathrm{C}(13)-\mathrm{P}(2)-\mathrm{C}(17)-\mathrm{C}(18) & 41.7(2) \\ \mathrm{Co}(1)-\mathrm{P}(1)-\mathrm{C}(7)-\mathrm{C}(9) & -178.92(16) & \mathrm{Co}(1)-\mathrm{P}(2)-\mathrm{C}(17)-\mathrm{C}(18) & -176.08(14) \\ \mathrm{C}(2)-\mathrm{P}(1)-\mathrm{C}(7)-\mathrm{C}(10) & -165.16(19) & \mathrm{C}(12)-\mathrm{P}(2)-\mathrm{C}(17)-\mathrm{C}(20) & 52.07(19) \\ \mathrm{C}(3)-\mathrm{P}(1)-\mathrm{C}(7)-\mathrm{C}(10) & 85.0(2) & \mathrm{C}(13)-\mathrm{P}(2)-\mathrm{C}(17)-\mathrm{C}(20) & 162.49(17) \\ \mathrm{Co}(1)-\mathrm{P}(1)-\mathrm{C}(7)-\mathrm{C}(10) & -56.2(2) & \mathrm{Co}(1)-\mathrm{P}(2)-\mathrm{C}(17)-\mathrm{C}(20) & -55.31(18)\end{array}$


2.2 X-ray Single-Crystal Structure Analysis of 6a.

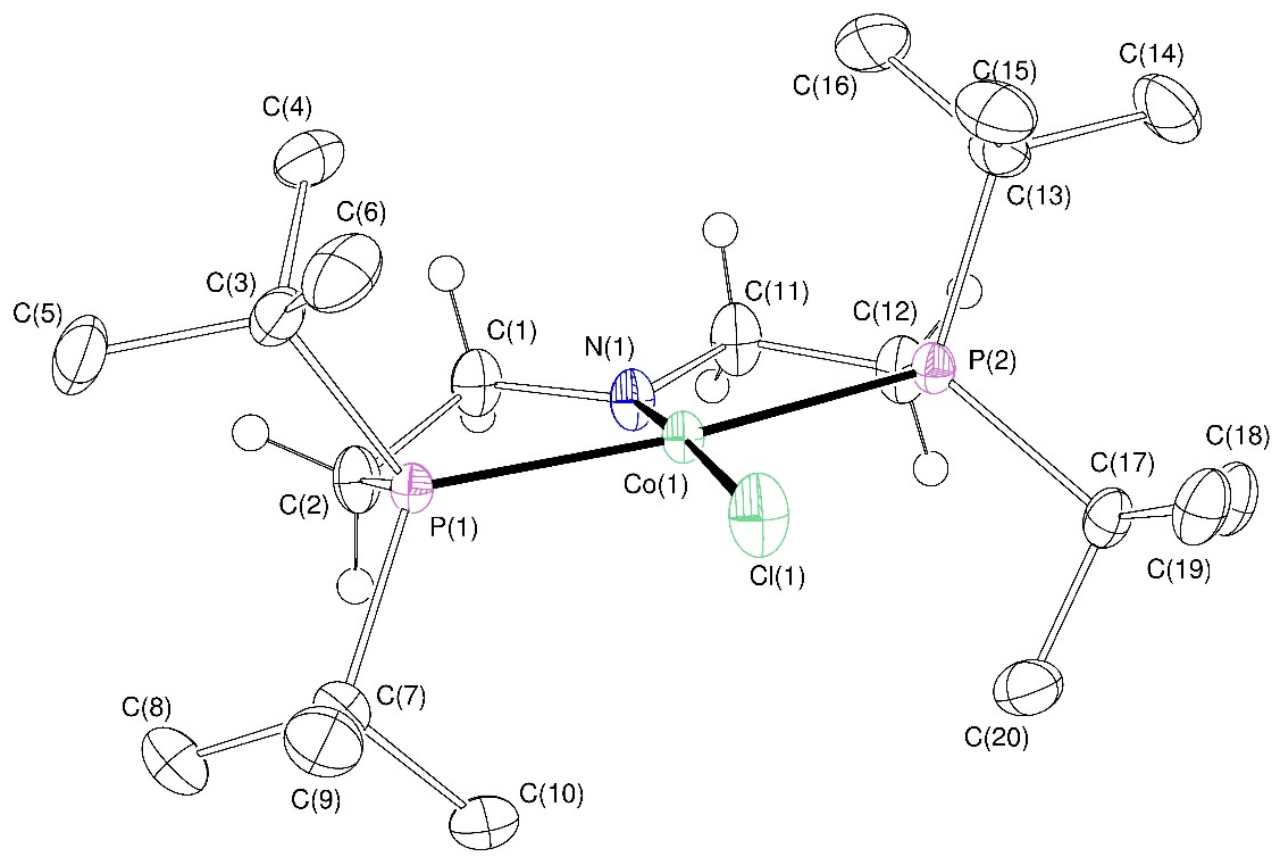

Figure S3. Thermal ellipsoid plot of $\mathbf{6 a}$ with the anisotropic displacement parameters drawn at the $50 \%$ probability level. All hydrogen atoms of the $t \mathrm{Bu}$-groups are omitted for clarity.

Table S4. Crystal data and structure refinement for $\mathbf{6 a}$.

\section{CCDC-No.}

Diffractometer and Detector

Identification code

Empirical formula

Formula weight

Temperature

Wavelength

Crystal system

Space group

Unit cell dimensions

Volume

Z

Density (calculated)

Absorption coefficient

$\mathrm{F}(000)$

Crystal shape and color:

Crystal size
1452221

Bruker, CMOS

mo_CW_PL_250215_0m_a

$\mathrm{C}_{20} \mathrm{H}_{44} \mathrm{ClCoNP}_{2}$

454.88

173(2) K

$0.71073 \AA$

Monoclinic

$\mathrm{P} 2{ }_{1} / \mathrm{c}$

$\mathrm{a}=12.1821(6) \AA$

$\alpha=90^{\circ}$

$\mathrm{b}=14.1820(6) \AA$

$\beta=104.471(2)^{\circ}$

$\mathrm{c}=14.4863(7) \AA$

$\gamma=90^{\circ}$

2423.3(2) $\AA^{3}$

4

$1.247 \mathrm{Mg} / \mathrm{m}^{3}$

$0.955 \mathrm{~mm}^{-1}$

980

Plate,

green

$0.368 \times 0.311 \times 0.141 \mathrm{~mm}^{3}$ 
Theta range for data collection

Index ranges

Reflections collected

Independent reflections

Completeness to theta $=25.242^{\circ}$

Absorption correction

Max. and min. transmission

Refinement method

Data / restraints / parameters

Goodness-of-fit on $\mathrm{F}^{2}$

Final R indices [I $>2 \operatorname{sigma}(\mathrm{I})]$

$\mathrm{R}$ indices (all data)

Largest diff. peak and hole

Table S5. Bond lengths $[\AA]$ and angles $\left[{ }^{\circ}\right]$ for $\mathbf{6 a}$.
2.246 to $30.584^{\circ}$

$-17<=\mathrm{h}<=17,-20<=\mathrm{k}<=20,-20<=\mathrm{l}<=20$

48653

$7439[\mathrm{R}($ int $)=0.0703]$

$99.9 \%$

Semi-empirical from equivalents

0.7461 and 0.6764

Full-matrix least-squares on $\mathrm{F}^{2}$

$7439 / 0 / 238$

1.041

$\mathrm{R} 1=0.0372, \mathrm{wR} 2=0.0664$

$\mathrm{R} 1=0.0699, \mathrm{wR} 2=0.0760$

0.384 and -0.358 e $\AA^{-3}$

$\begin{array}{ll}\mathrm{C}(1)-\mathrm{N}(1) & 1.462(2) \\ \mathrm{C}(1)-\mathrm{C}(2) & 1.518(2) \\ \mathrm{Cl}(1)-\mathrm{Co}(1) & 2.2291(5) \\ \mathrm{Co}(1)-\mathrm{N}(1) & 1.8337(13) \\ \mathrm{Co}(1)-\mathrm{P}(2) & 2.2156(5) \\ \mathrm{Co}(1)-\mathrm{P}(1) & 2.2200(5) \\ \mathrm{C}(2)-\mathrm{P}(1) & 1.8342(18) \\ \mathrm{C}(3)-\mathrm{C}(5) & 1.528(3) \\ \mathrm{C}(3)-\mathrm{C}(6) & 1.531(3) \\ \mathrm{C}(3)-\mathrm{C}(4) & 1.537(3) \\ \mathrm{C}(3)-\mathrm{P}(1) & 1.8768(18) \\ \mathrm{C}(7)-\mathrm{C}(9) & 1.531(3) \\ \mathrm{C}(7)-\mathrm{C}(8) & 1.537(3) \\ \mathrm{C}(7)-\mathrm{C}(10) & 1.539(3) \\ \mathrm{C}(7)-\mathrm{P}(1) & 1.8801(18) \\ \mathrm{C}(11)-\mathrm{N}(1) & 1.460(2) \\ \mathrm{C}(11)-\mathrm{C}(12) & 1.519(2) \\ \mathrm{C}(12)-\mathrm{P}(2) & 1.8269(18) \\ \mathrm{C}(13)-\mathrm{C}(15) & 1.532(3) \\ \mathrm{C}(13)-\mathrm{C}(14) & 1.532(3) \\ \mathrm{C}(13)-\mathrm{C}(16) & 1.536(3) \\ \mathrm{C}(13)-\mathrm{P}(2) & 1.8795(18) \\ \mathrm{C}(17)-\mathrm{C}(19) & 1.531(3) \\ \mathrm{C}(17)-\mathrm{C}(20) & 1.539(3) \\ & \end{array}$

$\mathrm{C}(6)-\mathrm{C}(3)-\mathrm{P}(1)$

C(4)-C(3)-P(1)

$\mathrm{C}(9)-\mathrm{C}(7)-\mathrm{C}(8)$

$\mathrm{C}(9)-\mathrm{C}(7)-\mathrm{C}(10)$

$\mathrm{C}(8)-\mathrm{C}(7)-\mathrm{C}(10)$

C(9)-C(7)-P(1)

C(8)-C(7)-P(1)

C(10)-C(7)-P(1)

$\mathrm{N}(1)-\mathrm{C}(11)-\mathrm{C}(12)$

$\mathrm{C}(11)-\mathrm{C}(12)-\mathrm{P}(2)$

C(15)-C(13)-C(14)

$\mathrm{C}(15)-\mathrm{C}(13)-\mathrm{C}(16)$

C(14)-C(13)-C(16)

$\mathrm{C}(15)-\mathrm{C}(13)-\mathrm{P}(2)$

C(14)-C(13)-P(2)

$\mathrm{C}(16)-\mathrm{C}(13)-\mathrm{P}(2)$

C(19)-C(17)-C(20)

$\mathrm{C}(19)-\mathrm{C}(17)-\mathrm{C}(18)$

$\mathrm{C}(20)-\mathrm{C}(17)-\mathrm{C}(18)$

C(19)-C(17)-P(2)

$\mathrm{C}(20)-\mathrm{C}(17)-\mathrm{P}(2)$

C(18)-C(17)-P(2)

$\mathrm{C}(11)-\mathrm{N}(1)-\mathrm{C}(1)$

$\mathrm{C}(11)-\mathrm{N}(1)-\mathrm{Co}(1)$

s9
109.84(12)

106.39(12)

109.10(16)

108.32(16)

108.72(16)

112.08(13)

113.45(14)

104.94(12)

111.33(14)

108.02(12)

110.57(16)

107.80(17)

108.09(16)

109.72(13)

113.76(14)

106.65(13)

108.56(15)

109.13(15)

108.61(15)

111.52(12)

105.55(12)

113.29(12)

108.21(13)

125.12(11) 


$\begin{array}{lcll}\mathrm{C}(17)-\mathrm{C}(18) & 1.540(2) & \mathrm{C}(1)-\mathrm{N}(1)-\mathrm{Co}(1) & 124.08(11) \\ \mathrm{C}(17)-\mathrm{P}(2) & 1.8827(17) & \mathrm{C}(2)-\mathrm{P}(1)-\mathrm{C}(3) & 105.40(8) \\ \mathrm{N}(1)-\mathrm{C}(1)-\mathrm{C}(2) & 110.82(14) & \mathrm{C}(2)-\mathrm{P}(1)-\mathrm{C}(7) & 103.42(9) \\ \mathrm{N}(1)-\mathrm{Co}(1)-\mathrm{P}(2) & 85.80(5) & \mathrm{C}(3)-\mathrm{P}(1)-\mathrm{C}(7) & 111.15(8) \\ \mathrm{N}(1)-\mathrm{Co}(1)-\mathrm{P}(1) & 86.26(5) & \mathrm{C}(2)-\mathrm{P}(1)-\mathrm{Co}(1) & 100.74(6) \\ \mathrm{P}(2)-\mathrm{Co}(1)-\mathrm{P}(1) & 171.519(18) & \mathrm{C}(3)-\mathrm{P}(1)-\mathrm{Co}(1) & 115.88(6) \\ \mathrm{N}(1)-\mathrm{Co}(1)-\mathrm{Cl}(1) & 174.90(5) & \mathrm{C}(7)-\mathrm{P}(1)-\mathrm{Co}(1) & 117.92(6) \\ \mathrm{P}(2)-\mathrm{Co}(1)-\mathrm{Cl}(1) & 94.310(18) & \mathrm{C}(12)-\mathrm{P}(2)-\mathrm{C}(13) & 105.97(9) \\ \mathrm{P}(1)-\mathrm{Co}(1)-\mathrm{Cl}(1) & 93.864(17) & \mathrm{C}(12)-\mathrm{P}(2)-\mathrm{C}(17) & 103.45(8) \\ \mathrm{C}(1)-\mathrm{C}(2)-\mathrm{P}(1) & 108.28(12) & \mathrm{C}(13)-\mathrm{P}(2)-\mathrm{C}(17) & 111.26(8) \\ \mathrm{C}(5)-\mathrm{C}(3)-\mathrm{C}(6) & 109.74(16) & \mathrm{C}(12)-\mathrm{P}(2)-\mathrm{Co}(1) & 101.09(6) \\ \mathrm{C}(5)-\mathrm{C}(3)-\mathrm{C}(4) & 108.65(15) & \mathrm{C}(13)-\mathrm{P}(2)-\mathrm{Co}(1) & 114.48(6) \\ \mathrm{C}(6)-\mathrm{C}(3)-\mathrm{C}(4) & 107.38(16) & \mathrm{C}(17)-\mathrm{P}(2)-\mathrm{Co}(1) & 118.52(6) \\ \mathrm{C}(5)-\mathrm{C}(3)-\mathrm{P}(1) & 114.55(13) & & \end{array}$

Table S6. Torsion angles $\left[^{\circ}\right]$ for $\mathbf{6 a}$.

$\begin{array}{lccc}\text { N(1)-C(1)-C(2)-P(1) } & 33.47(18) & \mathrm{C}(8)-\mathrm{C}(7)-\mathrm{P}(1)-\mathrm{C}(3) & 74.19(16) \\ \mathrm{N}(1)-\mathrm{C}(11)-\mathrm{C}(12)-\mathrm{P}(2) & -31.5(2) & \mathrm{C}(10)-\mathrm{C}(7)-\mathrm{P}(1)-\mathrm{C}(3) & -167.27(13) \\ \mathrm{C}(12)-\mathrm{C}(11)-\mathrm{N}(1)-\mathrm{C}(1) & -173.02(16) & \mathrm{C}(9)-\mathrm{C}(7)-\mathrm{P}(1)-\mathrm{Co}(1) & 87.35(14) \\ \mathrm{C}(12)-\mathrm{C}(11)-\mathrm{N}(1)-\mathrm{Co}(1) & 24.7(2) & \mathrm{C}(8)-\mathrm{C}(7)-\mathrm{P}(1)-\mathrm{Co}(1) & -148.53(12) \\ \mathrm{C}(2)-\mathrm{C}(1)-\mathrm{N}(1)-\mathrm{C}(11) & 166.34(16) & \mathrm{C}(10)-\mathrm{C}(7)-\mathrm{P}(1)-\mathrm{Co}(1) & -29.99(15) \\ \mathrm{C}(2)-\mathrm{C}(1)-\mathrm{N}(1)-\mathrm{Co}(1) & -31.2(2) & \mathrm{C}(11)-\mathrm{C}(12)-\mathrm{P}(2)-\mathrm{C}(13) & -93.88(14) \\ \mathrm{P}(2)-\mathrm{Co}(1)-\mathrm{N}(1)-\mathrm{C}(11) & -5.53(14) & \mathrm{C}(11)-\mathrm{C}(12)-\mathrm{P}(2)-\mathrm{C}(17) & 148.98(13) \\ \mathrm{P}(1)-\mathrm{Co}(1)-\mathrm{N}(1)-\mathrm{C}(11) & 171.49(15) & \mathrm{C}(11)-\mathrm{C}(12)-\mathrm{P}(2)-\mathrm{Co}(1) & 25.81(14) \\ \mathrm{P}(2)-\mathrm{Co}(1)-\mathrm{N}(1)-\mathrm{C}(1) & -165.08(14) & \mathrm{C}(15)-\mathrm{C}(13)-\mathrm{P}(2)-\mathrm{C}(12) & 167.05(13) \\ \mathrm{P}(1)-\mathrm{Co}(1)-\mathrm{N}(1)-\mathrm{C}(1) & 11.93(14) & \mathrm{C}(14)-\mathrm{C}(13)-\mathrm{P}(2)-\mathrm{C}(12) & -68.51(15) \\ \mathrm{C}(1)-\mathrm{C}(2)-\mathrm{P}(1)-\mathrm{C}(3) & 97.00(14) & \mathrm{C}(16)-\mathrm{C}(13)-\mathrm{P}(2)-\mathrm{C}(12) & 50.56(15) \\ \mathrm{C}(1)-\mathrm{C}(2)-\mathrm{P}(1)-\mathrm{C}(7) & -146.23(13) & \mathrm{C}(15)-\mathrm{C}(13)-\mathrm{P}(2)-\mathrm{C}(17) & -81.18(15) \\ \mathrm{C}(1)-\mathrm{C}(2)-\mathrm{P}(1)-\mathrm{Co}(1) & -23.87(13) & \mathrm{C}(14)-\mathrm{C}(13)-\mathrm{P}(2)-\mathrm{C}(17) & 43.26(16) \\ \mathrm{C}(5)-\mathrm{C}(3)-\mathrm{P}(1)-\mathrm{C}(2) & 68.58(15) & \mathrm{C}(16)-\mathrm{C}(13)-\mathrm{P}(2)-\mathrm{C}(17) & 162.33(13) \\ \mathrm{C}(6)-\mathrm{C}(3)-\mathrm{P}(1)-\mathrm{C}(2) & -167.40(13) & \mathrm{C}(15)-\mathrm{C}(13)-\mathrm{P}(2)-\mathrm{Co}(1) & 56.56(14) \\ \mathrm{C}(4)-\mathrm{C}(3)-\mathrm{P}(1)-\mathrm{C}(2) & -51.48(15) & \mathrm{C}(14)-\mathrm{C}(13)-\mathrm{P}(2)-\mathrm{Co}(1) & -179.00(12) \\ \mathrm{C}(5)-\mathrm{C}(3)-\mathrm{P}(1)-\mathrm{C}(7) & -42.81(16) & \mathrm{C}(16)-\mathrm{C}(13)-\mathrm{P}(2)-\mathrm{Co}(1) & -59.94(15) \\ \mathrm{C}(6)-\mathrm{C}(3)-\mathrm{P}(1)-\mathrm{C}(7) & 81.22(15) & \mathrm{C}(19)-\mathrm{C}(17)-\mathrm{P}(2)-\mathrm{C}(12) & 164.12(13) \\ \mathrm{C}(4)-\mathrm{C}(3)-\mathrm{P}(1)-\mathrm{C}(7) & -162.86(13) & \mathrm{C}(20)-\mathrm{C}(17)-\mathrm{P}(2)-\mathrm{C}(12) & -78.18(13) \\ \mathrm{C}(5)-\mathrm{C}(3)-\mathrm{P}(1)-\mathrm{Co}(1) & 178.97(12) & \mathrm{C}(18)-\mathrm{C}(17)-\mathrm{P}(2)-\mathrm{C}(12) & 40.54(15) \\ \mathrm{C}(6)-\mathrm{C}(3)-\mathrm{P}(1)-\mathrm{Co}(1) & -57.00(14) & \mathrm{C}(19)-\mathrm{C}(17)-\mathrm{P}(2)-\mathrm{C}(13) & 50.76(15) \\ \mathrm{C}(4)-\mathrm{C}(3)-\mathrm{P}(1)-\mathrm{Co}(1) & 58.92(14) & \mathrm{C}(20)-\mathrm{C}(17)-\mathrm{P}(2)-\mathrm{C}(13) & 168.46(12) \\ \mathrm{C}(9)-\mathrm{C}(7)-\mathrm{P}(1)-\mathrm{C}(2) & -162.57(13) & \mathrm{C}(18)-\mathrm{C}(17)-\mathrm{P}(2)-\mathrm{C}(13) & -72.83(15) \\ & & \mathrm{S} 10 & \\ & & & \\ & & & \\ & & & \end{array}$




$\begin{array}{llll}\mathrm{C}(8)-\mathrm{C}(7)-\mathrm{P}(1)-\mathrm{C}(2) & -38.46(16) & \mathrm{C}(19)-\mathrm{C}(17)-\mathrm{P}(2)-\mathrm{Co}(1) & -85.08(13) \\ \mathrm{C}(10)-\mathrm{C}(7)-\mathrm{P}(1)-\mathrm{C}(2) & 80.08(14) & \mathrm{C}(20)-\mathrm{C}(17)-\mathrm{P}(2)-\mathrm{Co}(1) & 32.62(14) \\ \mathrm{C}(9)-\mathrm{C}(7)-\mathrm{P}(1)-\mathrm{C}(3) & -49.93(15) & \mathrm{C}(18)-\mathrm{C}(17)-\mathrm{P}(2)-\mathrm{Co}(1) & 151.33(11)\end{array}$


2.3 X-ray Single-Crystal Structure Analysis of $\mathbf{6 b}$.

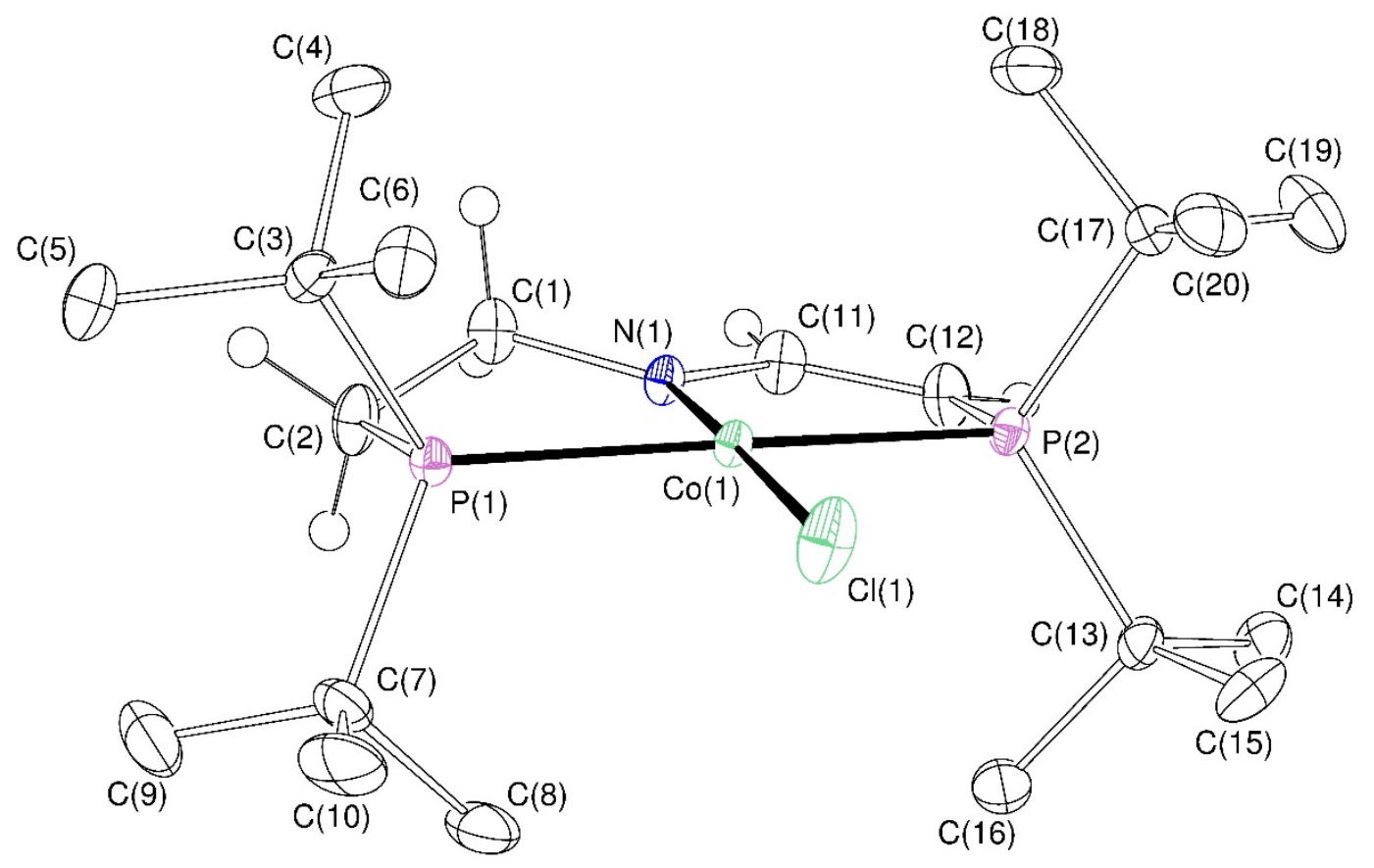

Figure S4. Thermal ellipsoid plot of $\mathbf{6 b}$ with the anisotropic displacement parameters drawn at the $50 \%$ probability level. All hydrogen atoms of the $t$ Bu-groups are omitted for clarity. The reflections 020 and 011 are removed from the refinement using OMIT command.

Table S7. Crystal data and structure refinement for $6 \mathbf{b}$.

CCDC-No.

Diffractometer and Detector

Identification code

Empirical formula

Formula weight

Temperature

Wavelength

Crystal system

Space group

Unit cell dimensions

Volume

Z

Density (calculated)

Absorption coefficient

$\mathrm{F}(000)$
1452222

Bruker, CCD

cw87abs_a

$\mathrm{C}_{20} \mathrm{H}_{42} \mathrm{ClCoNP}_{2}$

452.86

100(2) K

$0.56086 \AA$

Monoclinic

$\mathrm{P} 2 / \mathrm{c}$

$\mathrm{a}=8.3107(11) \AA$

$\alpha=90^{\circ}$

$\mathrm{b}=20.977(3) \AA$

$\beta=107.679(7)^{\circ}$

$\mathrm{c}=14.3233(16) \AA$

$\gamma=90^{\circ}$

4

$1.264 \mathrm{Mg} / \mathrm{m}^{3}$

$0.506 \mathrm{~mm}^{-1}$

972 
Crystal shape and color:

Crystal size

Theta range for data collection

Index ranges

Reflections collected

Independent reflections

Completeness to theta $=19.665^{\circ}$

Absorption correction

Max. and min. transmission

Refinement method

Data / restraints / parameters

Goodness-of-fit on $\mathrm{F}^{2}$

Final $\mathrm{R}$ indices $[\mathrm{I}>2 \operatorname{sigma}(\mathrm{I})]$

$\mathrm{R}$ indices (all data)

Largest diff. peak and hole

Table S7. Bond lengths $[\AA]$ and angles $\left[{ }^{\circ}\right]$ for $\mathbf{6 b}$.

$\begin{array}{ll}\mathrm{Co}(1)-\mathrm{N}(1) & 1.8646(15) \\ \mathrm{Co}(1)-\mathrm{Cl}(1) & 2.2118(5) \\ \mathrm{Co}(1)-\mathrm{P}(1) & 2.2410(6) \\ \mathrm{Co}(1)-\mathrm{P}(2) & 2.2508(6) \\ \mathrm{P}(1)-\mathrm{C}(2) & 1.8269(19) \\ \mathrm{P}(1)-\mathrm{C}(7) & 1.8822(19) \\ \mathrm{P}(1)-\mathrm{C}(3) & 1.8829(19) \\ \mathrm{P}(2)-\mathrm{C}(12) & 1.7914(19) \\ \mathrm{P}(2)-\mathrm{C}(17) & 1.8794(19) \\ \mathrm{P}(2)-\mathrm{C}(13) & 1.8817(19) \\ \mathrm{N}(1)-\mathrm{C}(11) & 1.387(2) \\ \mathrm{N}(1)-\mathrm{C}(1) & 1.448(2) \\ \mathrm{C}(1)-\mathrm{C}(2) & 1.476(3) \\ \mathrm{C}(3)-\mathrm{C}(5) & 1.531(3) \\ \mathrm{C}(3)-\mathrm{C}(4) & 1.536(3) \\ \mathrm{C}(3)-\mathrm{C}(6) & 1.539(3) \\ \mathrm{C}(7)-\mathrm{C}(9) & 1.533(3) \\ \mathrm{C}(7)-\mathrm{C}(10) & 1.534(3) \\ \mathrm{C}(7)-\mathrm{C}(8) & 1.544(3) \\ \mathrm{C}(11)-\mathrm{C}(12) & 1.370(3) \\ \mathrm{C}(13)-\mathrm{C}(15) & 1.528(3) \\ & \\ & \end{array}$

Block,

red

$0.252 \times 0.157 \times 0.095 \mathrm{~mm}^{3}$

1.932 to $22.133^{\circ}$

$-11<=\mathrm{h}<=11,-28<=\mathrm{k}<=27,-19<=\mathrm{l}<=19$

97587

$5999[\mathrm{R}($ int $)=0.1491]$

$100.0 \%$

Semi-empirical from equivalents

0.7447 and 0.6168

Full-matrix least-squares on $\mathrm{F}^{2}$

$5999 / 0 / 238$

0.956

$\begin{array}{ll}\mathrm{R} 1=0.0326, & \mathrm{wR} 2=0.0774 \\ \mathrm{R} 1=0.0534, & \mathrm{wR} 2=0.0851\end{array}$

0.419 and $-0.404 \mathrm{e}^{-3}$

105.48(9)

105.07(9)

112.95(8)

99.45(7)

114.91(6)

116.75(6)

115.46(15)

121.51(12)

122.66(12)

112.67(16)

109.32(13)

108.80(16)

109.69(15)

108.02(16)

114.27(13)

105.34(12)

110.45(13)

109.80(17)

108.83(17)

107.86(18)

$113.38(15)$ 


$\begin{array}{lrll}\mathrm{C}(13)-\mathrm{C}(14) & 1.534(3) & \mathrm{C}(10)-\mathrm{C}(7)-\mathrm{P}(1) & 111.53(14) \\ \mathrm{C}(13)-\mathrm{C}(16) & 1.537(3) & \mathrm{C}(8)-\mathrm{C}(7)-\mathrm{P}(1) & 105.17(13) \\ \mathrm{C}(17)-\mathrm{C}(19) & 1.530(3) & \mathrm{C}(12)-\mathrm{C}(11)-\mathrm{N}(1) & 121.10(17) \\ \mathrm{C}(17)-\mathrm{C}(20) & 1.533(3) & \mathrm{C}(11)-\mathrm{C}(12)-\mathrm{P}(2) & 111.76(14) \\ \mathrm{C}(17)-\mathrm{C}(18) & 1.534(3) & \mathrm{C}(15)-\mathrm{C}(13)-\mathrm{C}(14) & 108.97(16) \\ \mathrm{N}(1)-\mathrm{Co}(1)-\mathrm{Cl}(1) & 178.00(5) & \mathrm{C}(15)-\mathrm{C}(13)-\mathrm{C}(16) & 108.67(18) \\ \mathrm{N}(1)-\mathrm{Co}(1)-\mathrm{P}(1) & 85.57(5) & \mathrm{C}(14)-\mathrm{C}(13)-\mathrm{C}(16) & 108.34(17) \\ \mathrm{Cl}(1)-\mathrm{Co}(1)-\mathrm{P}(1) & 94.54(2) & \mathrm{C}(15)-\mathrm{C}(13)-\mathrm{P}(2) & 112.86(13) \\ \mathrm{N}(1)-\mathrm{Co}(1)-\mathrm{P}(2) & 85.25(5) & \mathrm{C}(14)-\mathrm{C}(13)-\mathrm{P}(2) & 113.01(14) \\ \mathrm{Cl}(1)-\mathrm{Co}(1)-\mathrm{P}(2) & 94.51(2) & \mathrm{C}(16)-\mathrm{C}(13)-\mathrm{P}(2) & 104.75(13) \\ \mathrm{P}(1)-\mathrm{Co}(1)-\mathrm{P}(2) & 170.17(2) & \mathrm{C}(19)-\mathrm{C}(17)-\mathrm{C}(20) & 109.55(17) \\ \mathrm{C}(2)-\mathrm{P}(1)-\mathrm{C}(7) & 103.66(9) & \mathrm{C}(19)-\mathrm{C}(17)-\mathrm{C}(18) & 108.84(18) \\ \mathrm{C}(2)-\mathrm{P}(1)-\mathrm{C}(3) & 106.44(9) & \mathrm{C}(20)-\mathrm{C}(17)-\mathrm{C}(18) & 108.18(17) \\ \mathrm{C}(7)-\mathrm{P}(1)-\mathrm{C}(3) & 111.81(8) & \mathrm{C}(19)-\mathrm{C}(17)-\mathrm{P}(2) & 113.77(14) \\ \mathrm{C}(2)-\mathrm{P}(1)-\mathrm{Co}(1) & 100.42(6) & \mathrm{C}(20)-\mathrm{C}(17)-\mathrm{P}(2) & 110.98(13) \\ \mathrm{C}(7)-\mathrm{P}(1)-\mathrm{Co}(1) & 114.11(7) & \mathrm{C}(18)-\mathrm{C}(17)-\mathrm{P}(2) & 105.28(13) \\ \mathrm{C}(3)-\mathrm{P}(1)-\mathrm{Co}(1) & 118.25(6) & & \end{array}$

Table S9. Torsion angles $\left[{ }^{\circ}\right]$ for $\mathbf{6 b}$.

$\begin{array}{lclc}\mathrm{P}(1)-\mathrm{Co}(1)-\mathrm{N}(1)-\mathrm{C}(11) & -173.67(14) & \mathrm{C}(3)-\mathrm{P}(1)-\mathrm{C}(7)-\mathrm{C}(8) & -166.23(14) \\ \mathrm{P}(2)-\mathrm{Co}(1)-\mathrm{N}(1)-\mathrm{C}(11) & 2.80(14) & \mathrm{Co}(1)-\mathrm{P}(1)-\mathrm{C}(7)-\mathrm{C}(8) & -28.72(16) \\ \mathrm{P}(1)-\mathrm{Co}(1)-\mathrm{N}(1)-\mathrm{C}(1) & 13.66(14) & \mathrm{C}(1)-\mathrm{N}(1)-\mathrm{C}(11)-\mathrm{C}(12) & 176.91(18) \\ \mathrm{P}(2)-\mathrm{Co}(1)-\mathrm{N}(1)-\mathrm{C}(1) & -169.86(15) & \mathrm{Co}(1)-\mathrm{N}(1)-\mathrm{C}(11)-\mathrm{C}(12) & 3.7(3) \\ \mathrm{C}(11)-\mathrm{N}(1)-\mathrm{C}(1)-\mathrm{C}(2) & 154.68(17) & \mathrm{N}(1)-\mathrm{C}(11)-\mathrm{C}(12)-\mathrm{P}(2) & -9.7(3) \\ \mathrm{Co}(1)-\mathrm{N}(1)-\mathrm{C}(1)-\mathrm{C}(2) & -32.2(2) & \mathrm{C}(17)-\mathrm{P}(2)-\mathrm{C}(12)-\mathrm{C}(11) & -109.55(16) \\ \mathrm{N}(1)-\mathrm{C}(1)-\mathrm{C}(2)-\mathrm{P}(1) & 33.0(2) & \mathrm{C}(13)-\mathrm{P}(2)-\mathrm{C}(12)-\mathrm{C}(11) & 130.88(16) \\ \mathrm{C}(7)-\mathrm{P}(1)-\mathrm{C}(2)-\mathrm{C}(1) & -139.70(15) & \mathrm{Co}(1)-\mathrm{P}(2)-\mathrm{C}(12)-\mathrm{C}(11) & 9.73(16) \\ \mathrm{C}(3)-\mathrm{P}(1)-\mathrm{C}(2)-\mathrm{C}(1) & 102.25(15) & \mathrm{C}(12)-\mathrm{P}(2)-\mathrm{C}(13)-\mathrm{C}(15) & 168.53(15) \\ \mathrm{Co}(1)-\mathrm{P}(1)-\mathrm{C}(2)-\mathrm{C}(1) & -21.53(15) & \mathrm{C}(17)-\mathrm{P}(2)-\mathrm{C}(13)-\mathrm{C}(15) & 54.07(17) \\ \mathrm{C}(2)-\mathrm{P}(1)-\mathrm{C}(3)-\mathrm{C}(5) & 68.97(15) & \mathrm{Co}(1)-\mathrm{P}(2)-\mathrm{C}(13)-\mathrm{C}(15) & -82.44(15) \\ \mathrm{C}(7)-\mathrm{P}(1)-\mathrm{C}(3)-\mathrm{C}(5) & -43.56(16) & \mathrm{C}(12)-\mathrm{P}(2)-\mathrm{C}(13)-\mathrm{C}(14) & 44.32(16) \\ \mathrm{Co}(1)-\mathrm{P}(1)-\mathrm{C}(3)-\mathrm{C}(5) & -179.15(11) & \mathrm{C}(17)-\mathrm{P}(2)-\mathrm{C}(13)-\mathrm{C}(14) & -70.14(16) \\ \mathrm{C}(2)-\mathrm{P}(1)-\mathrm{C}(3)-\mathrm{C}(4) & -50.39(15) & \mathrm{Co}(1)-\mathrm{P}(2)-\mathrm{C}(13)-\mathrm{C}(14) & 153.35(12) \\ \mathrm{C}(7)-\mathrm{P}(1)-\mathrm{C}(3)-\mathrm{C}(4) & -162.92(13) & \mathrm{C}(12)-\mathrm{P}(2)-\mathrm{C}(13)-\mathrm{C}(16) & -73.42(15) \\ \mathrm{Co}(1)-\mathrm{P}(1)-\mathrm{C}(3)-\mathrm{C}(4) & 61.49(14) & \mathrm{C}(17)-\mathrm{P}(2)-\mathrm{C}(13)-\mathrm{C}(16) & 172.12(13) \\ \mathrm{C}(2)-\mathrm{P}(1)-\mathrm{C}(3)-\mathrm{C}(6) & -166.79(13) & \mathrm{Co}(1)-\mathrm{P}(2)-\mathrm{C}(13)-\mathrm{C}(16) & 35.61(15) \\ \mathrm{C}(7)-\mathrm{P}(1)-\mathrm{C}(3)-\mathrm{C}(6) & 80.67(15) & \mathrm{C}(12)-\mathrm{P}(2)-\mathrm{C}(17)-\mathrm{C}(19) & -61.89(17) \\ \mathrm{Co}(1)-\mathrm{P}(1)-\mathrm{C}(3)-\mathrm{C}(6) & -54.92(14) & \mathrm{C}(13)-\mathrm{P}(2)-\mathrm{C}(17)-\mathrm{C}(19) & 52.33(18) \\ \mathrm{C}(2)-\mathrm{P}(1)-\mathrm{C}(7)-\mathrm{C}(9) & -39.28(16) & \mathrm{Co}(1)-\mathrm{P}(2)-\mathrm{C}(17)-\mathrm{C}(19) & -170.33(13) \\ & & \mathrm{S} 14 & \\ & & & \\ & & & \end{array}$




$\begin{array}{lccc}\mathrm{C}(3)-\mathrm{P}(1)-\mathrm{C}(7)-\mathrm{C}(9) & 74.98(16) & \mathrm{C}(12)-\mathrm{P}(2)-\mathrm{C}(17)-\mathrm{C}(20) & 174.02(14) \\ \mathrm{Co}(1)-\mathrm{P}(1)-\mathrm{C}(7)-\mathrm{C}(9) & -147.50(13) & \mathrm{C}(13)-\mathrm{P}(2)-\mathrm{C}(17)-\mathrm{C}(20) & -71.77(16) \\ \mathrm{C}(2)-\mathrm{P}(1)-\mathrm{C}(7)-\mathrm{C}(10) & -163.85(15) & \mathrm{Co}(1)-\mathrm{P}(2)-\mathrm{C}(17)-\mathrm{C}(20) & 65.57(14) \\ \mathrm{C}(3)-\mathrm{P}(1)-\mathrm{C}(7)-\mathrm{C}(10) & -49.58(17) & \mathrm{C}(12)-\mathrm{P}(2)-\mathrm{C}(17)-\mathrm{C}(18) & 57.19(16) \\ \mathrm{Co}(1)-\mathrm{P}(1)-\mathrm{C}(7)-\mathrm{C}(10) & 87.94(15) & \mathrm{C}(13)-\mathrm{P}(2)-\mathrm{C}(17)-\mathrm{C}(18) & 171.41(14) \\ \mathrm{C}(2)-\mathrm{P}(1)-\mathrm{C}(7)-\mathrm{C}(8) & 79.50(15) & \mathrm{Co}(1)-\mathrm{P}(2)-\mathrm{C}(17)-\mathrm{C}(18) & -51.25(15)\end{array}$




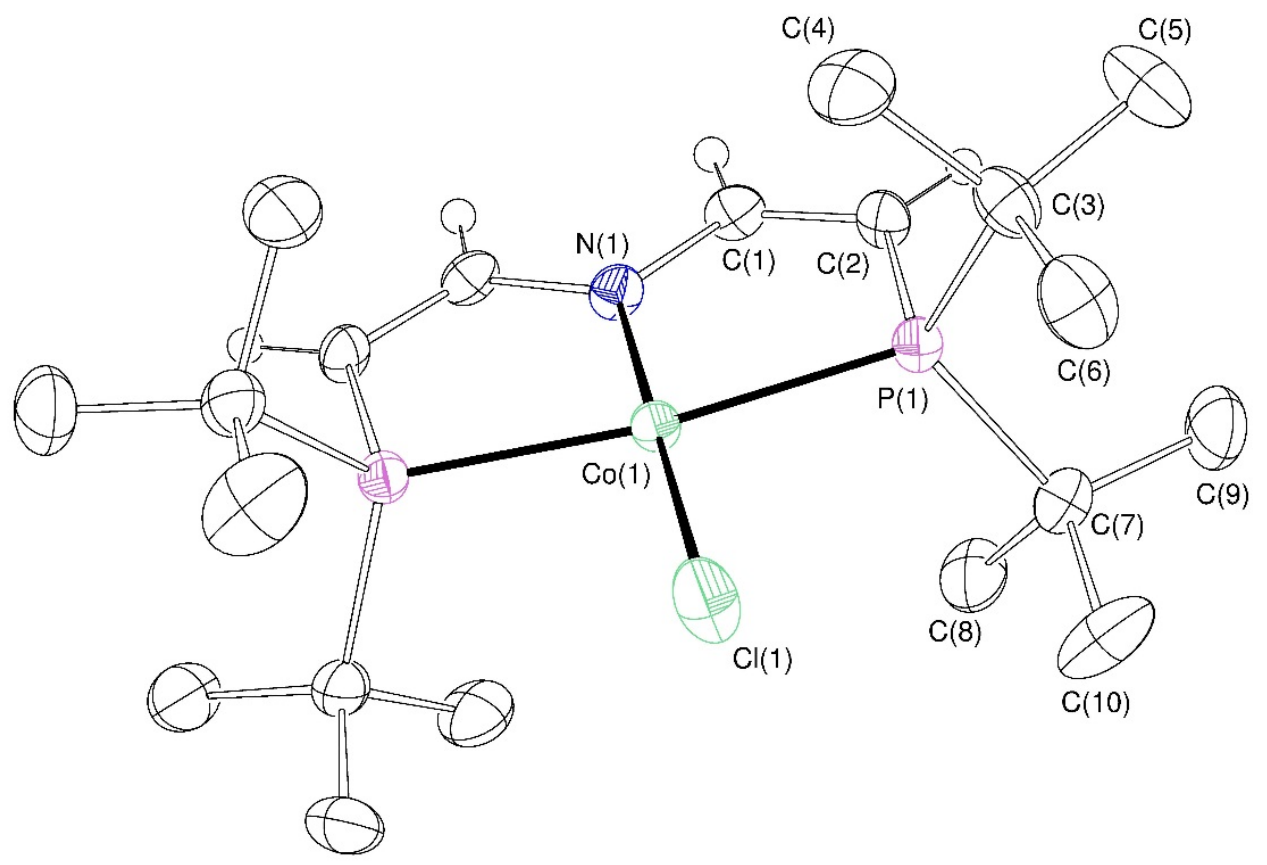

Figure S5. Thermal ellipsoid plot of $6 \mathbf{c}$ with the anisotropic displacement parameters drawn at the 50\% probability level. The asymmetric unit contains a half complex molecule. All hydrogen atoms of the $t \mathrm{Bu}$-groups are omitted for clarity.

Table S10. Crystal data and structure refinement for $\mathbf{6 c}$.

CCDC-No.

Diffractometer and Detector

Identification code

Empirical formula

Formula weight

Temperature

Wavelength

Crystal system

Space group

Unit cell dimensions

Volume

Z

Density (calculated)

Absorption coefficient

$\mathrm{F}(000)$

Crystal shape and color
1452223

Stoe, IPDS

pvlb_182

$\mathrm{C}_{20} \mathrm{H}_{40} \mathrm{ClCoNP}_{2}$

450.85

133(2) K

$0.71073 \AA$

Monoclinic

$\mathrm{P} 2 / \mathrm{c}$

$a=11.4745(4) \AA$

$\alpha=90^{\circ}$

$\mathrm{b}=8.3570(3) \AA$

$\beta=114.230(3)^{\circ}$

$\mathrm{c}=13.5663(6) \AA$

$\gamma=90^{\circ}$

1186.30(8) $\AA^{3}$

2

$1.262 \mathrm{Mg} / \mathrm{m}^{3}$

$0.975 \mathrm{~mm}^{-1}$

482

Plate,

red 
Crystal size

Theta range for data collection

Index ranges

Reflections collected

Independent reflections

Completeness to theta $=25.242^{\circ}$

Absorption correction

Max. and min. transmission

Refinement method

Data / restraints / parameters

Goodness-of-fit on $\mathrm{F}^{2}$

Final $\mathrm{R}$ indices [I $>2 \operatorname{sigma}(\mathrm{I})]$

$\mathrm{R}$ indices (all data)

Largest diff. peak and hole
$0.360 \times 0.060 \times 0.040 \mathrm{~mm}^{3}$

1.946 to $26.775^{\circ}$

$-14<=\mathrm{h}<=14,-9<=\mathrm{k}<=10,-16<=\mathrm{l}<=17$

10507

$2509[\mathrm{R}($ int $)=0.0545]$

$99.7 \%$

Numerical

0.9031 and 0.6416

Full-matrix least-squares on $\mathrm{F}^{2}$

$2509 / 0 / 121$

1.008

$\mathrm{R} 1=0.0328$,

$\mathrm{wR} 2=0.0720$

$\mathrm{R} 1=0.0448$,

$w R 2=0.0756$

0.422 and $-0.275 \mathrm{e}^{-3}$

Table S11. Bond lengths $[\AA]$ and angles $\left[^{\circ}\right]$ for $\mathbf{6 c}$.

\begin{tabular}{|c|c|c|c|}
\hline$C(1)-C(2)$ & $1.343(3)$ & $\mathrm{C}(5)-\mathrm{C}(3)-\mathrm{P}(1)$ & $112.87(15)$ \\
\hline $\mathrm{C}(1)-\mathrm{N}(1)$ & $1.382(2)$ & $\mathrm{C}(4)-\mathrm{C}(3)-\mathrm{P}(1)$ & $103.73(15)$ \\
\hline $\mathrm{C}(2)-\mathrm{P}(1)$ & $1.777(2)$ & $\mathrm{C}(10)-\mathrm{C}(7)-\mathrm{C}(9)$ & $110.2(2)$ \\
\hline$C(3)-C(6)$ & $1.526(3)$ & $\mathrm{C}(10)-\mathrm{C}(7)-\mathrm{C}(8)$ & $108.0(2)$ \\
\hline$C(3)-C(5)$ & $1.534(3)$ & $\mathrm{C}(9)-\mathrm{C}(7)-\mathrm{C}(8)$ & $108.4(2)$ \\
\hline$C(3)-C(4)$ & $1.537(3)$ & $\mathrm{C}(10)-\mathrm{C}(7)-\mathrm{P}(1)$ & $112.17(16)$ \\
\hline $\mathrm{C}(3)-\mathrm{P}(1)$ & $1.875(2)$ & $\mathrm{C}(9)-\mathrm{C}(7)-\mathrm{P}(1)$ & $113.15(16)$ \\
\hline$C(7)-C(10)$ & $1.530(3)$ & $\mathrm{C}(8)-\mathrm{C}(7)-\mathrm{P}(1)$ & $104.62(14)$ \\
\hline$C(7)-C(9)$ & $1.531(3)$ & $\mathrm{C}(1) \# 1-\mathrm{N}(1)-\mathrm{C}(1)$ & $118.3(2)$ \\
\hline$C(7)-C(8)$ & $1.533(3)$ & $\mathrm{C}(1) \# 1-\mathrm{N}(1)-\mathrm{Co}(1)$ & $120.86(11)$ \\
\hline $\mathrm{C}(7)-\mathrm{P}(1)$ & $1.877(2)$ & $\mathrm{C}(1)-\mathrm{N}(1)-\mathrm{Co}(1)$ & $120.86(11)$ \\
\hline $\mathrm{N}(1)-\mathrm{C}(1) \# 1$ & $1.382(2)$ & $\mathrm{C}(2)-\mathrm{P}(1)-\mathrm{C}(3)$ & $105.48(10)$ \\
\hline $\mathrm{N}(1)-\mathrm{Co}(1)$ & $1.893(2)$ & $\mathrm{C}(2)-\mathrm{P}(1)-\mathrm{C}(7)$ & $105.86(9)$ \\
\hline $\mathrm{P}(1)-\mathrm{Co}(1)$ & $2.2561(5)$ & $\mathrm{C}(3)-\mathrm{P}(1)-\mathrm{C}(7)$ & $113.54(10)$ \\
\hline $\mathrm{Cl}(1)-\mathrm{Co}(1)$ & $2.2026(7)$ & $\mathrm{C}(2)-\mathrm{P}(1)-\mathrm{Co}(1)$ & $99.39(7)$ \\
\hline $\operatorname{Co}(1)-\mathrm{P}(1) \# 1$ & $2.2562(5)$ & $\mathrm{C}(3)-\mathrm{P}(1)-\mathrm{Co}(1)$ & $112.91(7)$ \\
\hline $\mathrm{C}(2)-\mathrm{C}(1)-\mathrm{N}(1)$ & $121.47(18)$ & $\mathrm{C}(7)-\mathrm{P}(1)-\mathrm{Co}(1)$ & $117.55(7)$ \\
\hline $\mathrm{C}(1)-\mathrm{C}(2)-\mathrm{P}(1)$ & $113.52(14)$ & $\mathrm{N}(1)-\mathrm{Co}(1)-\mathrm{P}(1)$ & $84.756(14)$ \\
\hline$C(6)-C(3)-C(5)$ & $109.8(2)$ & $\mathrm{Cl}(1)-\mathrm{Co}(1)-\mathrm{P}(1)$ & $95.244(14)$ \\
\hline$C(6)-C(3)-C(4)$ & 109.27(19) & $\mathrm{N}(1)-\mathrm{Co}(1)-\mathrm{P}(1) \# 1$ & $84.755(14)$ \\
\hline$C(5)-C(3)-C(4)$ & $108.2(2)$ & $\mathrm{Cl}(1)-\mathrm{Co}(1)-\mathrm{P}(1) \# 1$ & $95.245(14)$ \\
\hline$C(6)-C(3)-P(1)$ & $112.64(15)$ & $\mathrm{P}(1)-\mathrm{Co}(1)-\mathrm{P}(1) \# 1$ & $169.51(3)$ \\
\hline
\end{tabular}


Table S12. Torsion angles $\left[{ }^{\circ}\right]$ for $\mathbf{6 c}$.

$\begin{array}{lclc}\mathrm{N}(1)-\mathrm{C}(1)-\mathrm{C}(2)-\mathrm{P}(1) & 0.4(2) & \mathrm{C}(4)-\mathrm{C}(3)-\mathrm{P}(1)-\mathrm{Co}(1) & -37.56(16) \\ \mathrm{C}(2)-\mathrm{C}(1)-\mathrm{N}(1)-\mathrm{C}(1) \# 1 & 179.8(2) & \mathrm{C}(10)-\mathrm{C}(7)-\mathrm{P}(1)-\mathrm{C}(2) & -177.09(18) \\ \mathrm{C}(2)-\mathrm{C}(1)-\mathrm{N}(1)-\mathrm{C}(1) & -0.2(2) & \mathrm{C}(9)-\mathrm{C}(7)-\mathrm{P}(1)-\mathrm{C}(2) & 57.52(19) \\ \mathrm{C}(1)-\mathrm{C}(2)-\mathrm{P}(1)-\mathrm{C}(3) & -117.40(16) & \mathrm{C}(8)-\mathrm{C}(7)-\mathrm{P}(1)-\mathrm{C}(2) & -60.27(17) \\ \mathrm{C}(1)-\mathrm{C}(2)-\mathrm{P}(1)-\mathrm{C}(7) & 121.98(16) & \mathrm{C}(10)-\mathrm{C}(7)-\mathrm{P}(1)-\mathrm{C}(3) & 67.7(2) \\ \mathrm{C}(1)-\mathrm{C}(2)-\mathrm{P}(1)-\mathrm{Co}(1) & -0.32(16) & \mathrm{C}(9)-\mathrm{C}(7)-\mathrm{P}(1)-\mathrm{C}(3) & -57.71(19) \\ \mathrm{C}(6)-\mathrm{C}(3)-\mathrm{P}(1)-\mathrm{C}(2) & -172.02(16) & \mathrm{C}(8)-\mathrm{C}(7)-\mathrm{P}(1)-\mathrm{C}(3) & -175.50(15) \\ \mathrm{C}(5)-\mathrm{C}(3)-\mathrm{P}(1)-\mathrm{C}(2) & -46.9(2) & \mathrm{C}(10)-\mathrm{C}(7)-\mathrm{P}(1)-\mathrm{Co}(1) & -67.24(19) \\ \mathrm{C}(4)-\mathrm{C}(3)-\mathrm{P}(1)-\mathrm{C}(2) & 69.95(16) & \mathrm{C}(9)-\mathrm{C}(7)-\mathrm{P}(1)-\mathrm{Co}(1) & 167.37(15) \\ \mathrm{C}(6)-\mathrm{C}(3)-\mathrm{P}(1)-\mathrm{C}(7) & -56.56(19) & \mathrm{C}(8)-\mathrm{C}(7)-\mathrm{P}(1)-\mathrm{Co}(1) & 49.58(17) \\ \mathrm{C}(5)-\mathrm{C}(3)-\mathrm{P}(1)-\mathrm{C}(7) & 68.5(2) & \mathrm{C}(1) \# 1-\mathrm{N}(1)-\mathrm{Co}(1)-\mathrm{P}(1) & 179.98(10) \\ \mathrm{C}(4)-\mathrm{C}(3)-\mathrm{P}(1)-\mathrm{C}(7) & -174.59(14) & \mathrm{C}(1)-\mathrm{N}(1)-\mathrm{Co}(1)-\mathrm{P}(1) & -0.02(10) \\ \mathrm{C}(6)-\mathrm{C}(3)-\mathrm{P}(1)-\mathrm{Co}(1) & 80.47(17) & \mathrm{C}(1) \# 1-\mathrm{N}(1)-\mathrm{Co}(1)-\mathrm{P}(1) \# 1 & -0.02(10) \\ \mathrm{C}(5)-\mathrm{C}(3)-\mathrm{P}(1)-\mathrm{Co}(1) & -154.45(16) & \mathrm{C}(1)-\mathrm{N}(1)-\mathrm{Co}(1)-\mathrm{P}(1) \# 1 & 179.98(10)\end{array}$




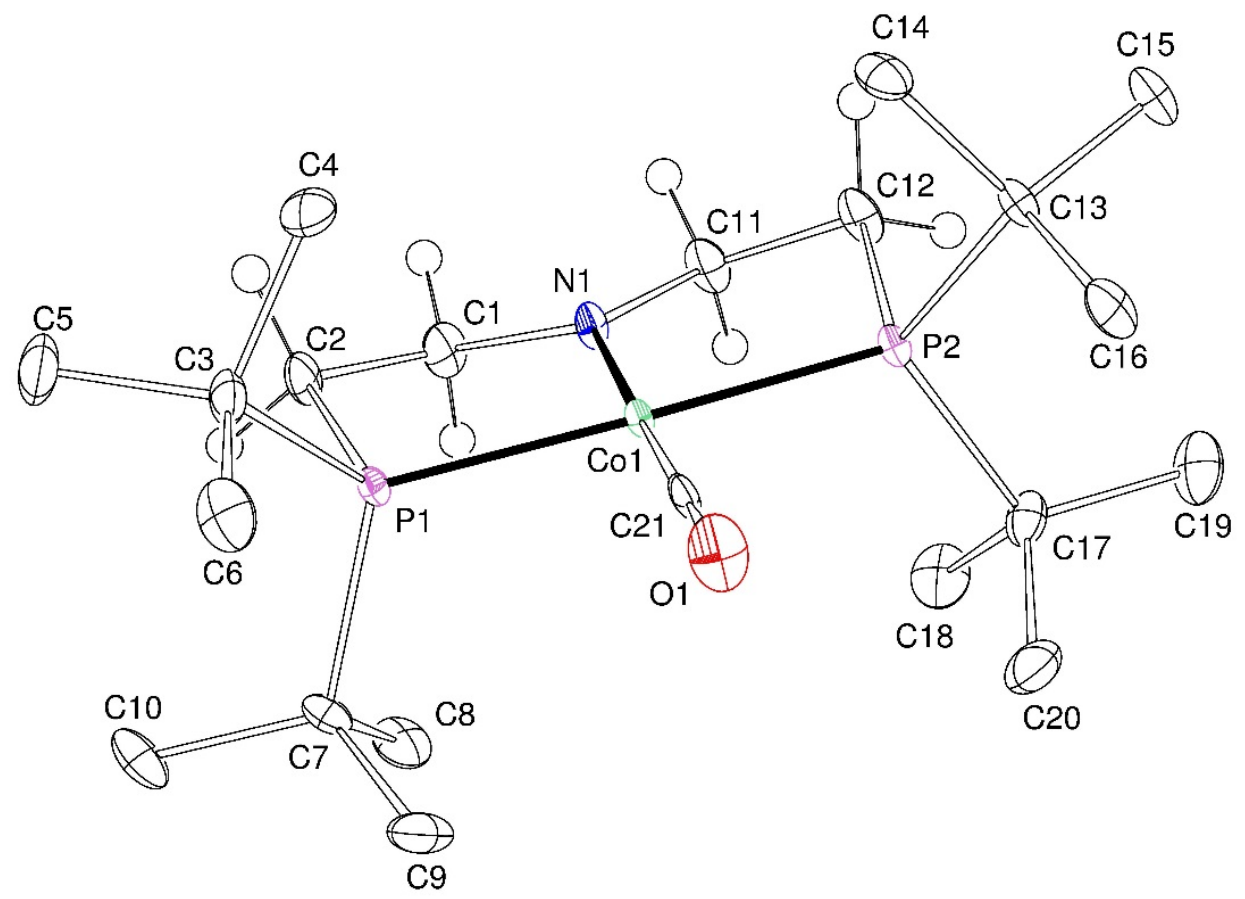

Figure S6. Thermal ellipsoid plot of $7 \mathbf{a}$ with the anisotropic displacement parameters drawn at the $50 \%$ probability level. All hydrogen atoms of the $t \mathrm{Bu}$-groups are omitted for clarity.

Table S13. Crystal data and structure refinement for $7 \mathbf{a}$.

CCDC-No.

Diffractometer and Detector

Identification code

Empirical formula

Formula weight

Temperature

Wavelength

Crystal system

Space group

Unit cell dimensions

Volume

Z

Density (calculated)

Absorption coefficient

$\mathrm{F}(000)$

Crystal shape and color:

Crystal size
1452371

Bruker, CMOS

mo_CW_BS_040216_0m_a

$\mathrm{C}_{21} \mathrm{H}_{44} \mathrm{CoNOP}_{2}$

447.44

104(2) K

$0.71073 \AA$

Monoclinic

$\mathrm{P} 2{ }_{1} / \mathrm{c}$

$\mathrm{a}=12.1068(9) \AA$

$\alpha=90^{\circ}$

$\mathrm{b}=14.3168(11) \AA$

$\beta=104.355(2)^{\circ}$

$\mathrm{c}=14.3964(11) \AA$

$\gamma=90^{\circ}$

2417.4(3) $\AA^{3}$

4

$1.229 \mathrm{Mg} / \mathrm{m}^{3}$

$0.852 \mathrm{~mm}^{-1}$

968

Plate,

brown

$0.168 \times 0.159 \times 0.044 \mathrm{~mm}^{3}$ 
Theta range for data collection

Index ranges

Reflections collected

Independent reflections

Completeness to theta $=25.242^{\circ}$

Absorption correction

Max. and min. transmission

Refinement method

Data / restraints / parameters

Goodness-of-fit on $\mathrm{F}^{2}$

Final R indices [I $>2 \operatorname{sigma}(\mathrm{I})]$

$\mathrm{R}$ indices (all data)

Largest diff. peak and hole
2.245 to $26.764^{\circ}$

$-15<=\mathrm{h}<=15,-18<=\mathrm{k}<=18,-18<=\mathrm{l}<=18$

38417

$5138[\mathrm{R}(\mathrm{int})=0.1104]$

$99.9 \%$

Semi-empirical from equivalents

0.7454 and 0.6484

Full-matrix least-squares on $\mathrm{F}^{2}$

$5138 / 0 / 247$

1.117

$\mathrm{R} 1=0.0486$,

$\mathrm{wR} 2=0.0853$

$\mathrm{R} 1=0.0731$,

$\mathrm{wR} 2=0.0918$

Table S14. Bond lengths $[\AA]$ and angles $\left[^{\circ}\right]$ for $7 \mathbf{a}$.

\begin{tabular}{|c|c|c|c|}
\hline $\mathrm{Co}(1)-\mathrm{C}(21)$ & $1.699(3)$ & $\mathrm{C}(1)-\mathrm{N}(1)-\mathrm{C}(11)$ & $109.3(2)$ \\
\hline $\mathrm{Co}(1)-\mathrm{N}(1)$ & $1.865(2)$ & $\mathrm{C}(1)-\mathrm{N}(1)-\mathrm{Co}(1)$ & $123.64(19)$ \\
\hline $\mathrm{Co}(1)-\mathrm{P}(2)$ & $2.1729(8)$ & $\mathrm{C}(11)-\mathrm{N}(1)-\mathrm{Co}(1)$ & 124.91(19) \\
\hline $\mathrm{Co}(1)-\mathrm{P}(1)$ & $2.1754(8)$ & $\mathrm{N}(1)-\mathrm{C}(1)-\mathrm{C}(2)$ & $111.3(2)$ \\
\hline$P(1)-C(2)$ & $1.836(3)$ & $\mathrm{C}(1)-\mathrm{C}(2)-\mathrm{P}(1)$ & $108.5(2)$ \\
\hline$P(1)-C(7)$ & $1.881(3)$ & $\mathrm{C}(12)-\mathrm{P}(2)-\mathrm{C}(17)$ & $106.01(14)$ \\
\hline$P(1)-C(3)$ & $1.886(3)$ & $\mathrm{C}(12)-\mathrm{P}(2)-\mathrm{C}(13)$ & $103.53(13)$ \\
\hline $\mathrm{O}(1)-\mathrm{C}(21)$ & $1.168(3)$ & $\mathrm{C}(17)-\mathrm{P}(2)-\mathrm{C}(13)$ & $110.87(13)$ \\
\hline $\mathrm{N}(1)-\mathrm{C}(1)$ & $1.454(4)$ & $\mathrm{C}(12)-\mathrm{P}(2)-\mathrm{Co}(1)$ & $102.10(10)$ \\
\hline $\mathrm{N}(1)-\mathrm{C}(11)$ & $1.454(4)$ & $\mathrm{C}(17)-\mathrm{P}(2)-\mathrm{Co}(1)$ & $114.61(10)$ \\
\hline $\mathrm{C}(1)-\mathrm{C}(2)$ & $1.504(4)$ & $\mathrm{C}(13)-\mathrm{P}(2)-\mathrm{Co}(1)$ & $117.89(9)$ \\
\hline $\mathrm{P}(2)-\mathrm{C}(12)$ & $1.834(3)$ & $\mathrm{C}(6)-\mathrm{C}(3)-\mathrm{C}(5)$ & $109.4(3)$ \\
\hline $\mathrm{P}(2)-\mathrm{C}(17)$ & $1.882(3)$ & $C(6)-C(3)-C(4)$ & $108.2(3)$ \\
\hline $\mathrm{P}(2)-\mathrm{C}(13)$ & $1.885(3)$ & $C(5)-C(3)-C(4)$ & $108.2(3)$ \\
\hline$C(3)-C(6)$ & $1.527(4)$ & $\mathrm{C}(6)-\mathrm{C}(3)-\mathrm{P}(1)$ & $112.1(2)$ \\
\hline$C(3)-C(5)$ & $1.536(4)$ & $\mathrm{C}(5)-\mathrm{C}(3)-\mathrm{P}(1)$ & $113.7(2)$ \\
\hline$C(3)-C(4)$ & $1.550(4)$ & $\mathrm{C}(4)-\mathrm{C}(3)-\mathrm{P}(1)$ & $104.9(2)$ \\
\hline$C(7)-C(10)$ & $1.527(4)$ & $C(10)-C(7)-C(9)$ & $110.2(3)$ \\
\hline$C(7)-C(9)$ & $1.532(4)$ & $\mathrm{C}(10)-\mathrm{C}(7)-\mathrm{C}(8)$ & $108.8(3)$ \\
\hline $\mathrm{C}(7)-\mathrm{C}(8)$ & $1.542(4)$ & $\mathrm{C}(9)-\mathrm{C}(7)-\mathrm{C}(8)$ & $107.7(3)$ \\
\hline $\mathrm{C}(11)-\mathrm{C}(12)$ & $1.519(4)$ & $C(10)-C(7)-P(1)$ & $114.4(2)$ \\
\hline$C(13)-C(16)$ & $1.534(4)$ & $\mathrm{C}(9)-\mathrm{C}(7)-\mathrm{P}(1)$ & $109.8(2)$ \\
\hline$C(13)-C(15)$ & $1.536(4)$ & $\mathrm{C}(8)-\mathrm{C}(7)-\mathrm{P}(1)$ & $105.6(2)$ \\
\hline \multirow[t]{2}{*}{$C(13)-C(14)$} & $1.537(4)$ & $\mathrm{N}(1)-\mathrm{C}(11)-\mathrm{C}(12)$ & $110.9(2)$ \\
\hline & \multicolumn{3}{|c|}{ S20 } \\
\hline
\end{tabular}




$\begin{array}{lcll}\mathrm{C}(17)-\mathrm{C}(19) & 1.527(4) & \mathrm{C}(11)-\mathrm{C}(12)-\mathrm{P}(2) & 108.1(2) \\ \mathrm{C}(17)-\mathrm{C}(18) & 1.537(4) & \mathrm{C}(16)-\mathrm{C}(13)-\mathrm{C}(15) & 108.9(2) \\ \mathrm{C}(17)-\mathrm{C}(20) & 1.538(4) & \mathrm{C}(16)-\mathrm{C}(13)-\mathrm{C}(14) & 108.4(2) \\ \mathrm{C}(21)-\mathrm{Co}(1)-\mathrm{N}(1) & 175.85(13) & \mathrm{C}(15)-\mathrm{C}(13)-\mathrm{C}(14) & 108.4(2) \\ \mathrm{C}(21)-\mathrm{Co}(1)-\mathrm{P}(2) & 94.23(10) & \mathrm{C}(16)-\mathrm{C}(13)-\mathrm{P}(2) & 111.18(19) \\ \mathrm{N}(1)-\mathrm{Co}(1)-\mathrm{P}(2) & 85.77(8) & \mathrm{C}(15)-\mathrm{C}(13)-\mathrm{P}(2) & 113.7(2) \\ \mathrm{C}(21)-\mathrm{Co}(1)-\mathrm{P}(1) & \mathrm{C}(14)-\mathrm{C}(13)-\mathrm{P}(2) & 106.02(19) \\ \mathrm{N}(1)-\mathrm{Co}(1)-\mathrm{P}(1) & 94.02(10) & \mathrm{C}(19)-\mathrm{C}(17)-\mathrm{C}(18) & 108.1(3) \\ \mathrm{P}(2)-\mathrm{Co}(1)-\mathrm{P}(1) & 86.15(8) & \mathrm{C}(19)-\mathrm{C}(17)-\mathrm{C}(20) & 110.3(3) \\ \mathrm{C}(2)-\mathrm{P}(1)-\mathrm{C}(7) & 171.53(3) & \mathrm{C}(18)-\mathrm{C}(17)-\mathrm{C}(20) & 107.6(3) \\ \mathrm{C}(2)-\mathrm{P}(1)-\mathrm{C}(3) & 105.87(14) & \mathrm{C}(19)-\mathrm{C}(17)-\mathrm{P}(2) & 114.5(2) \\ \mathrm{C}(7)-\mathrm{P}(1)-\mathrm{C}(3) & 103.60(14) & \mathrm{C}(18)-\mathrm{C}(17)-\mathrm{P}(2) & 106.2(2) \\ \mathrm{C}(2)-\mathrm{P}(1)-\mathrm{Co}(1) & 110.50(13) & \mathrm{C}(20)-\mathrm{C}(17)-\mathrm{P}(2) & 109.9(2) \\ \mathrm{C}(7)-\mathrm{P}(1)-\mathrm{Co}(1) & 101.99(10) & \mathrm{O}(1)-\mathrm{C}(21)-\mathrm{Co}(1) & 179.0(3) \\ \mathrm{C}(3)-\mathrm{P}(1)-\mathrm{Co}(1) & 115.77(10) & & \end{array}$

Table S15. Torsion angles $\left[^{\circ}\right]$ for $7 \mathbf{a}$.

\begin{tabular}{|c|c|c|c|}
\hline $\mathrm{P}(2)-\mathrm{Co}(1)-\mathrm{N}(1)-\mathrm{C}(1)$ & $166.8(2)$ & $\mathrm{C}(3)-\mathrm{P}(1)-\mathrm{C}(7)-\mathrm{C}(8)$ & $162.8(2)$ \\
\hline $\mathrm{P}(1)-\mathrm{Co}(1)-\mathrm{N}(1)-\mathrm{C}(1)$ & $-10.7(2)$ & $\mathrm{Co}(1)-\mathrm{P}(1)-\mathrm{C}(7)-\mathrm{C}(8)$ & $-60.9(2)$ \\
\hline $\mathrm{P}(2)-\mathrm{Co}(1)-\mathrm{N}(1)-\mathrm{C}(11)$ & $5.1(2)$ & $\mathrm{C}(1)-\mathrm{N}(1)-\mathrm{C}(11)-\mathrm{C}(12)$ & $172.3(3)$ \\
\hline $\mathrm{P}(1)-\mathrm{Co}(1)-\mathrm{N}(1)-\mathrm{C}(11)$ & $-172.4(2)$ & $\mathrm{Co}(1)-\mathrm{N}(1)-\mathrm{C}(11)-\mathrm{C}(12)$ & $-23.8(4)$ \\
\hline $\mathrm{C}(11)-\mathrm{N}(1)-\mathrm{C}(1)-\mathrm{C}(2)$ & $-167.2(3)$ & $\mathrm{N}(1)-\mathrm{C}(11)-\mathrm{C}(12)-\mathrm{P}(2)$ & $30.5(3)$ \\
\hline $\mathrm{Co}(1)-\mathrm{N}(1)-\mathrm{C}(1)-\mathrm{C}(2)$ & $28.7(3)$ & $\mathrm{C}(17)-\mathrm{P}(2)-\mathrm{C}(12)-\mathrm{C}(11)$ & $94.7(2)$ \\
\hline $\mathrm{N}(1)-\mathrm{C}(1)-\mathrm{C}(2)-\mathrm{P}(1)$ & $-31.1(3)$ & $\mathrm{C}(13)-\mathrm{P}(2)-\mathrm{C}(12)-\mathrm{C}(11)$ & $-148.5(2)$ \\
\hline $\mathrm{C}(7)-\mathrm{P}(1)-\mathrm{C}(2)-\mathrm{C}(1)$ & $-99.0(2)$ & $\mathrm{Co}(1)-\mathrm{P}(2)-\mathrm{C}(12)-\mathrm{C}(11)$ & $-25.6(2)$ \\
\hline $\mathrm{C}(3)-\mathrm{P}(1)-\mathrm{C}(2)-\mathrm{C}(1)$ & $144.7(2)$ & $\mathrm{C}(12)-\mathrm{P}(2)-\mathrm{C}(13)-\mathrm{C}(16)$ & $-165.0(2)$ \\
\hline $\mathrm{Co}(1)-\mathrm{P}(1)-\mathrm{C}(2)-\mathrm{C}(1)$ & $22.5(2)$ & $\mathrm{C}(17)-\mathrm{P}(2)-\mathrm{C}(13)-\mathrm{C}(16)$ & $-51.7(2)$ \\
\hline $\mathrm{C}(2)-\mathrm{P}(1)-\mathrm{C}(3)-\mathrm{C}(6)$ & $163.7(2)$ & $\mathrm{Co}(1)-\mathrm{P}(2)-\mathrm{C}(13)-\mathrm{C}(16)$ & $83.2(2)$ \\
\hline $\mathrm{C}(7)-\mathrm{P}(1)-\mathrm{C}(3)-\mathrm{C}(6)$ & $50.7(3)$ & $\mathrm{C}(12)-\mathrm{P}(2)-\mathrm{C}(13)-\mathrm{C}(15)$ & $-41.6(2)$ \\
\hline $\mathrm{Co}(1)-\mathrm{P}(1)-\mathrm{C}(3)-\mathrm{C}(6)$ & $-84.9(2)$ & $\mathrm{C}(17)-\mathrm{P}(2)-\mathrm{C}(13)-\mathrm{C}(15)$ & $71.7(2)$ \\
\hline $\mathrm{C}(2)-\mathrm{P}(1)-\mathrm{C}(3)-\mathrm{C}(5)$ & $38.8(3)$ & $\mathrm{Co}(1)-\mathrm{P}(2)-\mathrm{C}(13)-\mathrm{C}(15)$ & $-153.41(19)$ \\
\hline $\mathrm{C}(7)-\mathrm{P}(1)-\mathrm{C}(3)-\mathrm{C}(5)$ & $-74.2(3)$ & $\mathrm{C}(12)-\mathrm{P}(2)-\mathrm{C}(13)-\mathrm{C}(14)$ & $77.4(2)$ \\
\hline $\mathrm{Co}(1)-\mathrm{P}(1)-\mathrm{C}(3)-\mathrm{C}(5)$ & $150.2(2)$ & $\mathrm{C}(17)-\mathrm{P}(2)-\mathrm{C}(13)-\mathrm{C}(14)$ & $-169.33(19)$ \\
\hline $\mathrm{C}(2)-\mathrm{P}(1)-\mathrm{C}(3)-\mathrm{C}(4)$ & $-79.1(2)$ & $\mathrm{Co}(1)-\mathrm{P}(2)-\mathrm{C}(13)-\mathrm{C}(14)$ & $-34.4(2)$ \\
\hline $\mathrm{C}(7)-\mathrm{P}(1)-\mathrm{C}(3)-\mathrm{C}(4)$ & $167.9(2)$ & $\mathrm{C}(12)-\mathrm{P}(2)-\mathrm{C}(17)-\mathrm{C}(19)$ & $68.6(3)$ \\
\hline $\mathrm{Co}(1)-\mathrm{P}(1)-\mathrm{C}(3)-\mathrm{C}(4)$ & $32.3(2)$ & $\mathrm{C}(13)-\mathrm{P}(2)-\mathrm{C}(17)-\mathrm{C}(19)$ & $-43.1(3)$ \\
\hline $\mathrm{C}(2)-\mathrm{P}(1)-\mathrm{C}(7)-\mathrm{C}(10)$ & $-68.4(2)$ & $\mathrm{Co}(1)-\mathrm{P}(2)-\mathrm{C}(17)-\mathrm{C}(19)$ & $-179.62(19)$ \\
\hline $\mathrm{C}(3)-\mathrm{P}(1)-\mathrm{C}(7)-\mathrm{C}(10)$ & $43.2(3)$ & $\mathrm{C}(12)-\mathrm{P}(2)-\mathrm{C}(17)-\mathrm{C}(18)$ & $-50.6(2)$ \\
\hline $\mathrm{Co}(1)-\mathrm{P}(1)-\mathrm{C}(7)-\mathrm{C}(10)$ & $179.48(19)$ & $\mathrm{C}(13)-\mathrm{P}(2)-\mathrm{C}(17)-\mathrm{C}(18)$ & $-162.3(2)$ \\
\hline
\end{tabular}




$\begin{array}{llll}\mathrm{C}(2)-\mathrm{P}(1)-\mathrm{C}(7)-\mathrm{C}(9) & 167.1(2) & \mathrm{Co}(1)-\mathrm{P}(2)-\mathrm{C}(17)-\mathrm{C}(18) & 61.2(2) \\ \mathrm{C}(3)-\mathrm{P}(1)-\mathrm{C}(7)-\mathrm{C}(9) & -81.4(2) & \mathrm{C}(12)-\mathrm{P}(2)-\mathrm{C}(17)-\mathrm{C}(20) & -166.6(2) \\ \mathrm{Co}(1)-\mathrm{P}(1)-\mathrm{C}(7)-\mathrm{C}(9) & 54.9(2) & \mathrm{C}(13)-\mathrm{P}(2)-\mathrm{C}(17)-\mathrm{C}(20) & 81.7(2) \\ \mathrm{C}(2)-\mathrm{P}(1)-\mathrm{C}(7)-\mathrm{C}(8) & 51.3(2) & \mathrm{Co}(1)-\mathrm{P}(2)-\mathrm{C}(17)-\mathrm{C}(20) & -54.8(2)\end{array}$


2.6 X-ray Single-Crystal Structure Analysis of 8a.

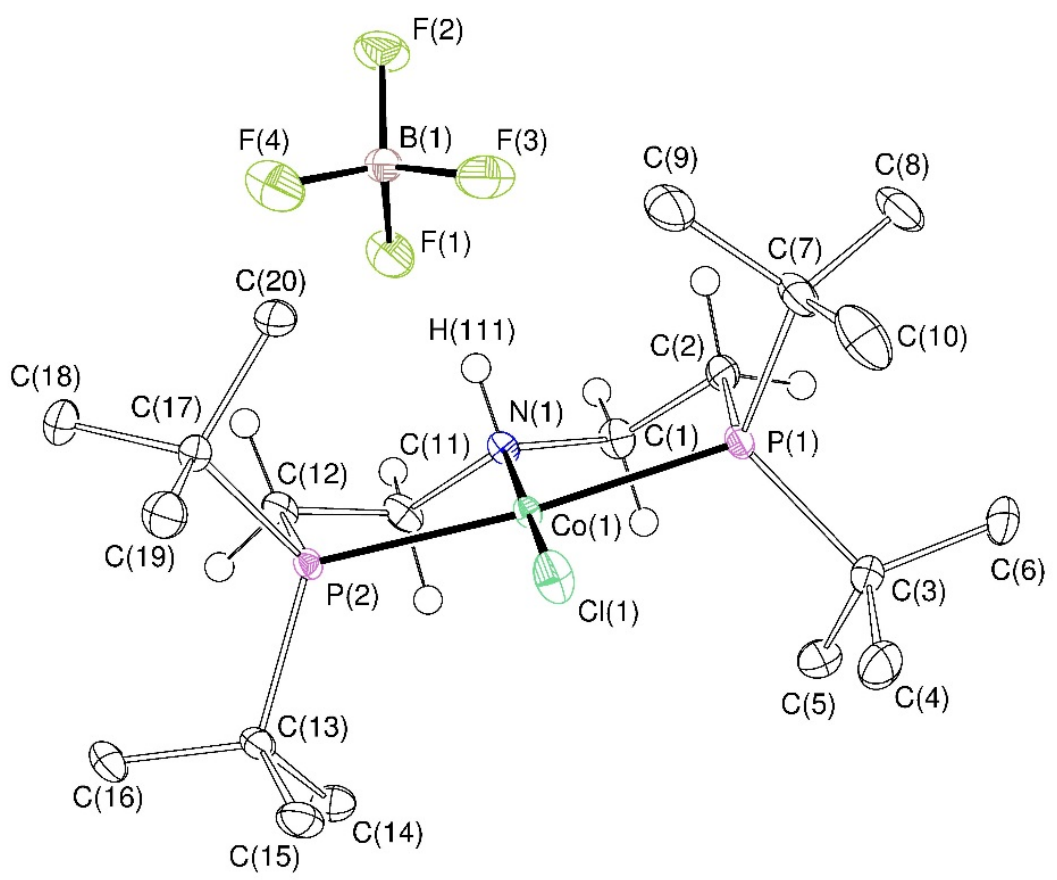

Figure S7. Thermal ellipsoid plot of 8a with the anisotropic displacement parameters drawn at the 50\% probability level. The N-H hydrogen atom was found and isotropically refined. All hydrogen atoms of the $t \mathrm{Bu}-$ groups are omitted for clarity.

Table S16. Crystal data and structure refinement for $\mathbf{8 a}$.

CCDC-No.

Diffractometer and Detector

Identification code

Empirical formula

Formula weight

Temperature

Wavelength

Crystal system

Space group

Unit cell dimensions

Volume

Z

Density (calculated)

Absorption coefficient

$\mathrm{F}(000)$
1452224

Bruker, CMOS

mo_CW_BS_241115_0m_a

$\mathrm{C}_{20} \mathrm{H}_{45} \mathrm{BClCoF}_{4} \mathrm{NP}_{2}$

542.70

100(2) K

$0.71073 \AA$

Monoclinic

$\mathrm{C} 2 / \mathrm{c}$

$\mathrm{a}=29.0335(9) \AA$

$\alpha=90^{\circ}$

$\mathrm{b}=14.0241(4) \AA$

$\beta=112.500(2)^{\circ}$

$\mathrm{c}=14.3063(5) \AA$ $\gamma=90^{\circ}$

5381.7(3) $\AA^{3}$

8

$1.340 \mathrm{Mg} / \mathrm{m}^{3}$

$0.892 \mathrm{~mm}^{-1}$

2296 
Crystal shape and color:

Crystal size

Theta range for data collection

Index ranges

Reflections collected

Independent reflections

Completeness to theta $=25.242^{\circ}$

Absorption correction

Max. and min. transmission

Refinement method

Data / restraints / parameters

Goodness-of-fit on $\mathrm{F}^{2}$

Final $\mathrm{R}$ indices [I $>2 \operatorname{sigma}(\mathrm{I})]$

$\mathrm{R}$ indices (all data)

Largest diff. peak and hole
Plate, red

$0.603 \times 0.407 \times 0.138 \mathrm{~mm}^{3}$

2.440 to $30.595^{\circ}$

$-41<=\mathrm{h}<=41,-20<=\mathrm{k}<=20,-20<=\mathrm{l}<=20$

83899

$8278[\mathrm{R}($ int $)=0.0702]$

$99.9 \%$

Semi-empirical from equivalents

0.7461 and 0.6098

Full-matrix least-squares on $\mathrm{F}^{2}$

8278 / 0 / 287

1.017

$\mathrm{R} 1=0.0314$,

$\mathrm{wR} 2=0.0655$

$\mathrm{R} 1=0.0444$,

$w R 2=0.0698$

Table S17. Bond lengths $[\AA]$ and angles $\left[^{\circ}\right]$ for $\mathbf{8 a}$.

$\begin{array}{ll}\mathrm{C}(1)-\mathrm{N}(1) & 1.4909(17) \\ \mathrm{C}(1)-\mathrm{C}(2) & 1.5148(19) \\ \mathrm{C}(2)-\mathrm{P}(1) & 1.8384(14) \\ \mathrm{C}(3)-\mathrm{C}(6) & 1.533(2) \\ \mathrm{C}(3)-\mathrm{C}(4) & 1.534(2) \\ \mathrm{C}(3)-\mathrm{C}(5) & 1.537(2) \\ \mathrm{C}(3)-\mathrm{P}(1) & 1.8750(14) \\ \mathrm{C}(7)-\mathrm{C}(10) & 1.534(2) \\ \mathrm{C}(7)-\mathrm{C}(9) & 1.537(2) \\ \mathrm{C}(7)-\mathrm{C}(8) & 1.537(2) \\ \mathrm{C}(7)-\mathrm{P}(1) & 1.8747(14) \\ \mathrm{C}(11)-\mathrm{N}(1) & 1.4933(16) \\ \mathrm{C}(11)-\mathrm{C}(12) & 1.517(2) \\ \mathrm{C}(12)-\mathrm{P}(2) & 1.8433(13) \\ \mathrm{C}(13)-\mathrm{C}(15) & 1.5343(18) \\ \mathrm{C}(13)-\mathrm{C}(16) & 1.5350(19) \\ \mathrm{C}(13)-\mathrm{C}(14) & 1.5365(19) \\ \mathrm{C}(13)-\mathrm{P}(2) & 1.8710(13) \\ \mathrm{C}(17)-\mathrm{C}(19) & 1.5281(19) \\ \mathrm{C}(17)-\mathrm{C}(18) & 1.5401(19) \\ \mathrm{C}(17)-\mathrm{C}(20) & 1.5411(18) \\ \mathrm{C}(17)-\mathrm{P}(2) & 1.8729(14) \\ & \\ & \end{array}$

$\begin{array}{ll}\mathrm{N}(1)-\mathrm{C}(11)-\mathrm{C}(12) & 108.92(11) \\ \mathrm{C}(11)-\mathrm{C}(12)-\mathrm{P}(2) & 108.20(9) \\ \mathrm{C}(15)-\mathrm{C}(13)-\mathrm{C}(16) & 110.11(11) \\ \mathrm{C}(15)-\mathrm{C}(13)-\mathrm{C}(14) & 107.74(11) \\ \mathrm{C}(16)-\mathrm{C}(13)-\mathrm{C}(14) & 108.93(11) \\ \mathrm{C}(15)-\mathrm{C}(13)-\mathrm{P}(2) & 110.95(9) \\ \mathrm{C}(16)-\mathrm{C}(13)-\mathrm{P}(2) & 112.42(9) \\ \mathrm{C}(14)-\mathrm{C}(13)-\mathrm{P}(2) & 106.50(9) \\ \mathrm{C}(19)-\mathrm{C}(17)-\mathrm{C}(18) & 109.76(11) \\ \mathrm{C}(19)-\mathrm{C}(17)-\mathrm{C}(20) & 108.63(11) \\ \mathrm{C}(18)-\mathrm{C}(17)-\mathrm{C}(20) & 108.62(11) \\ \mathrm{C}(19)-\mathrm{C}(17)-\mathrm{P}(2) & 111.29(9) \\ \mathrm{C}(18)-\mathrm{C}(17)-\mathrm{P}(2) & 113.39(10) \\ \mathrm{C}(20)-\mathrm{C}(17)-\mathrm{P}(2) & 104.92(9) \\ \mathrm{F}(2)-\mathrm{B}(1)-\mathrm{F}(4) & 110.66(13) \\ \mathrm{F}(2)-\mathrm{B}(1)-\mathrm{F}(1) & 111.13(13) \\ \mathrm{F}(4)-\mathrm{B}(1)-\mathrm{F}(1) & 107.59(12) \\ \mathrm{F}(2)-\mathrm{B}(1)-\mathrm{F}(3) & 110.13(13) \\ \mathrm{F}(4)-\mathrm{B}(1)-\mathrm{F}(3) & 107.74(14) \\ \mathrm{F}(1)-\mathrm{B}(1)-\mathrm{F}(3) & 109.49(13) \\ \mathrm{C}(1)-\mathrm{N}(1)-\mathrm{C}(11) & 109.93(10) \\ \mathrm{C}(1)-\mathrm{N}(1)-\mathrm{Co}(1) & 116.59(8) \\ & \end{array}$




$\begin{array}{llll}\mathrm{B}(1)-\mathrm{F}(2) & 1.3681(18) & \mathrm{C}(11)-\mathrm{N}(1)-\mathrm{Co}(1) & 114.50(8) \\ \mathrm{B}(1)-\mathrm{F}(4) & 1.391(2) & \mathrm{C}(1)-\mathrm{N}(1)-\mathrm{H}(111) & 105.4(11) \\ \mathrm{B}(1)-\mathrm{F}(1) & 1.3949(19) & \mathrm{C}(11)-\mathrm{N}(1)-\mathrm{H}(111) & 105.5(11) \\ \mathrm{B}(1)-\mathrm{F}(3) & 1.3957(19) & \mathrm{Co}(1)-\mathrm{N}(1)-\mathrm{H}(111) & 103.7(11) \\ \mathrm{N}(1)-\mathrm{Co}(1) & 1.9835(11) & \mathrm{C}(2)-\mathrm{P}(1)-\mathrm{C}(7) & 103.54(7) \\ \mathrm{N}(1)-\mathrm{H}(111) & 0.897(17) & \mathrm{C}(2)-\mathrm{P}(1)-\mathrm{C}(3) & 105.44(7) \\ \mathrm{P}(1)-\mathrm{Co}(1) & 2.2694(4) & \mathrm{C}(7)-\mathrm{P}(1)-\mathrm{C}(3) & 113.15(7) \\ \mathrm{P}(2)-\mathrm{Co}(1) & 2.2775(4) & \mathrm{C}(2)-\mathrm{P}(1)-\mathrm{Co}(1) & 100.37(4) \\ \mathrm{Cl}(1)-\mathrm{Co}(1) & 2.2045(4) & \mathrm{C}(7)-\mathrm{P}(1)-\mathrm{Co}(1) & 115.88(5) \\ \mathrm{N}(1)-\mathrm{C}(1)-\mathrm{C}(2) & 109.54(11) & \mathrm{C}(3)-\mathrm{P}(1)-\mathrm{Co}(1) & 116.03(5) \\ \mathrm{C}(1)-\mathrm{C}(2)-\mathrm{P}(1) & 108.75(9) & \mathrm{C}(12)-\mathrm{P}(2)-\mathrm{C}(13) & 105.16(6) \\ \mathrm{C}(6)-\mathrm{C}(3)-\mathrm{C}(4) & 109.92(12) & \mathrm{C}(12)-\mathrm{P}(2)-\mathrm{C}(17) & 104.66(6) \\ \mathrm{C}(6)-\mathrm{C}(3)-\mathrm{C}(5) & 107.92(13) & \mathrm{C}(13)-\mathrm{P}(2)-\mathrm{C}(17) & 113.14(6) \\ \mathrm{C}(4)-\mathrm{C}(3)-\mathrm{C}(5) & 108.04(12) & \mathrm{C}(12)-\mathrm{P}(2)-\mathrm{Co}(1) & 100.18(4) \\ \mathrm{C}(6)-\mathrm{C}(3)-\mathrm{P}(1) & 113.15(10) & \mathrm{C}(13)-\mathrm{P}(2)-\mathrm{Co}(1) & 118.66(4) \\ \mathrm{C}(4)-\mathrm{C}(3)-\mathrm{P}(1) & 110.50(10) & \mathrm{C}(17)-\mathrm{P}(2)-\mathrm{Co}(1) & 112.75(4) \\ \mathrm{C}(5)-\mathrm{C}(3)-\mathrm{P}(1) & 107.11(9) & \mathrm{N}(1)-\mathrm{Co}(1)-\mathrm{Cl}(1) & 176.08(4) \\ \mathrm{C}(10)-\mathrm{C}(7)-\mathrm{C}(9) & 108.92(14) & \mathrm{N}(1)-\mathrm{Co}(1)-\mathrm{P}(1) & 85.58(3) \\ \mathrm{C}(10)-\mathrm{C}(7)-\mathrm{C}(8) & 109.13(13) & \mathrm{Cl}(1)-\mathrm{Co}(1)-\mathrm{P}(1) & 94.443(14) \\ \mathrm{C}(9)-\mathrm{C}(7)-\mathrm{C}(8) & 108.56(13) & \mathrm{N}(1)-\mathrm{Co}(1)-\mathrm{P}(2) & 85.38(3) \\ \mathrm{C}(10)-\mathrm{C}(7)-\mathrm{P}(1) & 112.08(11) & \mathrm{Cl}(1)-\mathrm{Co}(1)-\mathrm{P}(2) & 94.445(14) \\ \mathrm{C}(9)-\mathrm{C}(7)-\mathrm{P}(1) & 105.57(10) & \mathrm{P}(1)-\mathrm{Co}(1)-\mathrm{P}(2) & 170.783(14) \\ \mathrm{C}(8)-\mathrm{C}(7)-\mathrm{P}(1) & 112.42(10) & & \end{array}$

Table S18. Torsion angles [ $\left.{ }^{\circ}\right]$ for $\mathbf{8 a}$.

$\begin{array}{lcll}\mathrm{N}(1)-\mathrm{C}(1)-\mathrm{C}(2)-\mathrm{P}(1) & 46.03(13) & \mathrm{C}(6)-\mathrm{C}(3)-\mathrm{P}(1)-\mathrm{Co}(1) & 175.35(9) \\ \mathrm{N}(1)-\mathrm{C}(11)-\mathrm{C}(12)-\mathrm{P}(2) & -48.06(12) & \mathrm{C}(4)-\mathrm{C}(3)-\mathrm{P}(1)-\mathrm{Co}(1) & -60.90(11) \\ \mathrm{C}(2)-\mathrm{C}(1)-\mathrm{N}(1)-\mathrm{C}(11) & -179.06(10) & \mathrm{C}(5)-\mathrm{C}(3)-\mathrm{P}(1)-\mathrm{Co}(1) & 56.55(11) \\ \mathrm{C}(2)-\mathrm{C}(1)-\mathrm{N}(1)-\mathrm{Co}(1) & -46.62(13) & \mathrm{C}(11)-\mathrm{C}(12)-\mathrm{P}(2)-\mathrm{C}(13) & -99.04(10) \\ \mathrm{C}(12)-\mathrm{C}(11)-\mathrm{N}(1)-\mathrm{C}(1) & -173.68(11) & \mathrm{C}(11)-\mathrm{C}(12)-\mathrm{P}(2)-\mathrm{C}(17) & 141.51(9) \\ \mathrm{C}(12)-\mathrm{C}(11)-\mathrm{N}(1)-\mathrm{Co}(1) & 52.80(13) & \mathrm{C}(11)-\mathrm{C}(12)-\mathrm{P}(2)-\mathrm{Co}(1) & 24.57(9) \\ \mathrm{C}(1)-\mathrm{C}(2)-\mathrm{P}(1)-\mathrm{C}(7) & -146.97(10) & \mathrm{C}(15)-\mathrm{C}(13)-\mathrm{P}(2)-\mathrm{C}(12) & 169.86(10) \\ \mathrm{C}(1)-\mathrm{C}(2)-\mathrm{P}(1)-\mathrm{C}(3) & 93.95(10) & \mathrm{C}(16)-\mathrm{C}(13)-\mathrm{P}(2)-\mathrm{C}(12) & -66.35(11) \\ \mathrm{C}(1)-\mathrm{C}(2)-\mathrm{P}(1)-\mathrm{Co}(1) & -26.94(10) & \mathrm{C}(14)-\mathrm{C}(13)-\mathrm{P}(2)-\mathrm{C}(12) & 52.88(10) \\ \mathrm{C}(10)-\mathrm{C}(7)-\mathrm{P}(1)-\mathrm{C}(2) & -163.66(11) & \mathrm{C}(15)-\mathrm{C}(13)-\mathrm{P}(2)-\mathrm{C}(17) & -76.50(11) \\ \mathrm{C}(9)-\mathrm{C}(7)-\mathrm{P}(1)-\mathrm{C}(2) & 77.90(11) & \mathrm{C}(16)-\mathrm{C}(13)-\mathrm{P}(2)-\mathrm{C}(17) & 47.29(11) \\ \mathrm{C}(8)-\mathrm{C}(7)-\mathrm{P}(1)-\mathrm{C}(2) & -40.29(13) & \mathrm{C}(14)-\mathrm{C}(13)-\mathrm{P}(2)-\mathrm{C}(17) & 166.52(9) \\ \mathrm{C}(10)-\mathrm{C}(7)-\mathrm{P}(1)-\mathrm{C}(3) & -50.02(13) & \mathrm{C}(15)-\mathrm{C}(13)-\mathrm{P}(2)-\mathrm{Co}(1) & 58.96(11) \\ \mathrm{C}(9)-\mathrm{C}(7)-\mathrm{P}(1)-\mathrm{C}(3) & -168.47(10) & \mathrm{C}(16)-\mathrm{C}(13)-\mathrm{P}(2)-\mathrm{Co}(1) & -177.25(8) \\ & & \mathrm{S} 25\end{array}$




$\begin{array}{lccc}\mathrm{C}(8)-\mathrm{C}(7)-\mathrm{P}(1)-\mathrm{C}(3) & 73.34(13) & \mathrm{C}(14)-\mathrm{C}(13)-\mathrm{P}(2)-\mathrm{Co}(1) & -58.02(10) \\ \mathrm{C}(10)-\mathrm{C}(7)-\mathrm{P}(1)-\mathrm{Co}(1) & 87.53(11) & \mathrm{C}(19)-\mathrm{C}(17)-\mathrm{P}(2)-\mathrm{C}(12) & 160.87(9) \\ \mathrm{C}(9)-\mathrm{C}(7)-\mathrm{P}(1)-\mathrm{Co}(1) & -30.92(12) & \mathrm{C}(18)-\mathrm{C}(17)-\mathrm{P}(2)-\mathrm{C}(12) & 36.55(11) \\ \mathrm{C}(8)-\mathrm{C}(7)-\mathrm{P}(1)-\mathrm{Co}(1) & -149.10(10) & \mathrm{C}(20)-\mathrm{C}(17)-\mathrm{P}(2)-\mathrm{C}(12) & -81.83(9) \\ \mathrm{C}(6)-\mathrm{C}(3)-\mathrm{P}(1)-\mathrm{C}(2) & 65.31(12) & \mathrm{C}(19)-\mathrm{C}(17)-\mathrm{P}(2)-\mathrm{C}(13) & 46.93(11) \\ \mathrm{C}(4)-\mathrm{C}(3)-\mathrm{P}(1)-\mathrm{C}(2) & -170.94(10) & \mathrm{C}(18)-\mathrm{C}(17)-\mathrm{P}(2)-\mathrm{C}(13) & -77.39(11) \\ \mathrm{C}(5)-\mathrm{C}(3)-\mathrm{P}(1)-\mathrm{C}(2) & -53.49(11) & \mathrm{C}(20)-\mathrm{C}(17)-\mathrm{P}(2)-\mathrm{C}(13) & 164.23(8) \\ \mathrm{C}(6)-\mathrm{C}(3)-\mathrm{P}(1)-\mathrm{C}(7) & -47.16(13) & \mathrm{C}(19)-\mathrm{C}(17)-\mathrm{P}(2)-\mathrm{Co}(1) & -91.20(9) \\ \mathrm{C}(4)-\mathrm{C}(3)-\mathrm{P}(1)-\mathrm{C}(7) & 76.58(11) & \mathrm{C}(18)-\mathrm{C}(17)-\mathrm{P}(2)-\mathrm{Co}(1) & 144.47(9) \\ \mathrm{C}(5)-\mathrm{C}(3)-\mathrm{P}(1)-\mathrm{C}(7) & -165.97(10) & \mathrm{C}(20)-\mathrm{C}(17)-\mathrm{P}(2)-\mathrm{Co}(1) & 26.10(9)\end{array}$


2.7 X-ray Single-Crystal Structure Analysis of 8c.
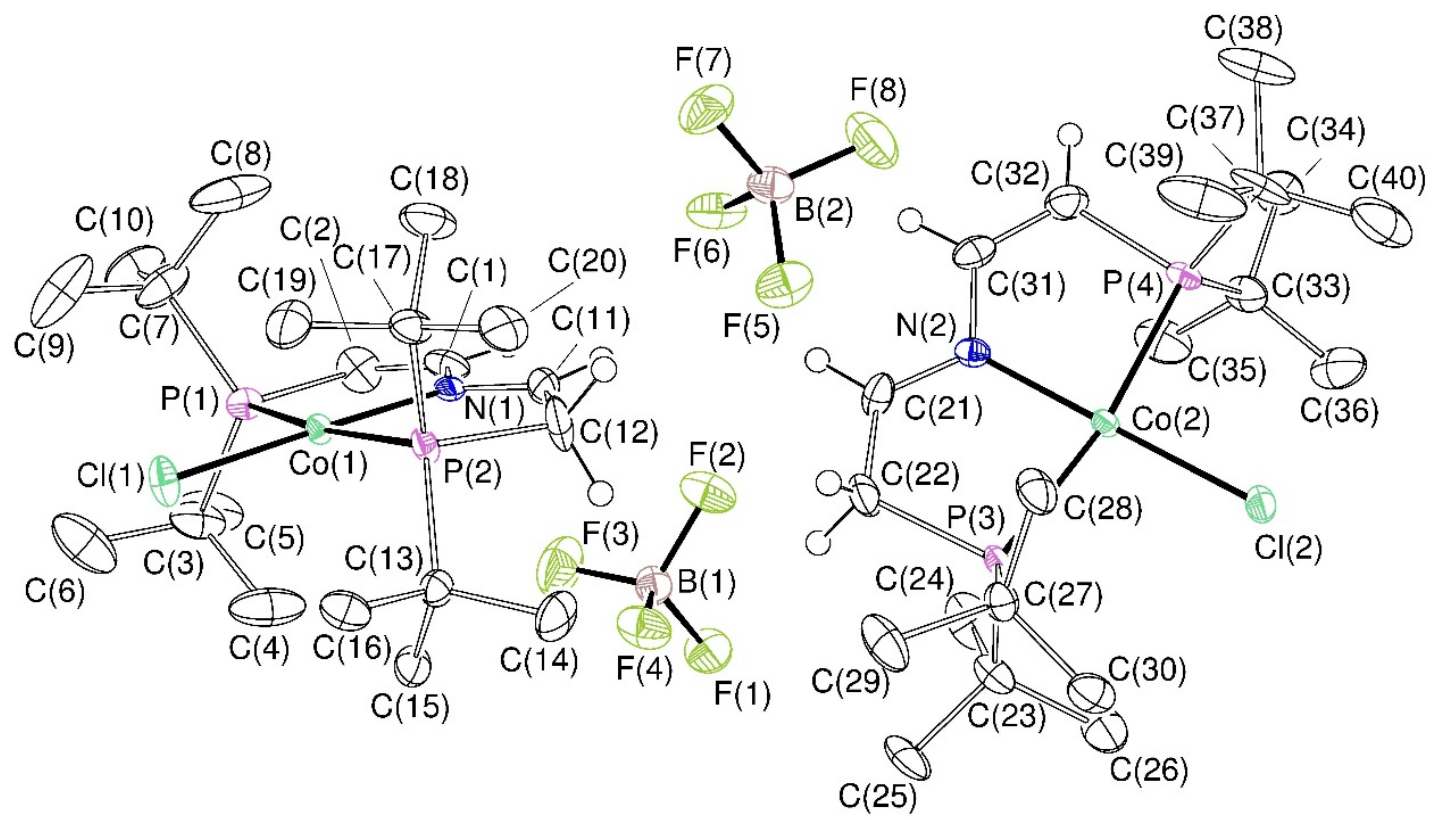

Figure S8. Thermal ellipsoid plot of 8c with the anisotropic displacement parameters drawn at the $50 \%$ probability level. The asymmetric unit contains two complex molecules and two $\mathrm{BF}_{4}$ anions. All hydrogen atoms of the $t \mathrm{Bu}$-groups are omitted for clarity.

Table S19. Crystal data and structure refinement for $\mathbf{8 c}$.

CCDC-No.

Diffractometer and Detector

Identification code

Empirical formula

Formula weight

Temperature

Wavelength

Crystal system

Space group

Unit cell dimensions

Volume

Z

Density (calculated)

Absorption coefficient

$\mathrm{F}(000)$

Crystal shape and color:

Crystal size
1452225

Bruker, CMOS

mo_CW_BS_091215_0m_a

$\mathrm{C}_{20} \mathrm{H}_{41} \mathrm{BClCoF}_{4} \mathrm{NP}_{2}$

538.67

101(2) K

$0.71073 \AA$

Triclinic

P-1

$$
\begin{array}{ll}
\mathrm{a}=13.8511(6) \AA & \alpha=113.737(2)^{\circ} \\
\mathrm{b}=13.8909(7) \AA & \beta=109.182(2)^{\circ} \\
\mathrm{c}=16.2469(8) \AA & \gamma=90.202(2)^{\circ}
\end{array}
$$

2669.1(2) $\AA^{3}$

4

$1.340 \mathrm{Mg} / \mathrm{m}^{3}$

$0.899 \mathrm{~mm}^{-1}$

1132

Plate, red

$0.246 \times 0.100 \times 0.032 \mathrm{~mm}^{3}$ 
Theta range for data collection

Index ranges

Reflections collected

Independent reflections

Completeness to theta $=25.242^{\circ}$

Absorption correction

Max. and min. transmission

Refinement method

Data / restraints / parameters

Goodness-of-fit on $\mathrm{F}^{2}$

Final R indices [I $>2 \operatorname{sigma}(\mathrm{I})]$

$\mathrm{R}$ indices (all data)

Largest diff. peak and hole
2.470 to $25.350^{\circ}$

$-16<=\mathrm{h}<=16,-16<=\mathrm{k}<=16,-19<=\mathrm{k}<=19$

65047

$9772[\mathrm{R}(\mathrm{int})=0.0771]$

$99.9 \%$

Semi-empirical from equivalents

0.7454 and 0.6302

Full-matrix least-squares on $\mathrm{F}^{2}$

9772 / 0 / 565

1.156

$\mathrm{R} 1=0.0668$,

$\mathrm{wR} 2=0.1395$

$\mathrm{R} 1=0.0889$,

$w R 2=0.1495$

Table S20. Bond lengths $[\AA]$ and angles $\left[^{\circ}\right]$ for $\mathbf{8 c}$.

\begin{tabular}{|c|c|c|c|}
\hline $\mathrm{C}(1)-\mathrm{C}(2)$ & $1.340(9)$ & $\mathrm{C}(19)-\mathrm{C}(17)-\mathrm{P}(2)$ & $110.5(4)$ \\
\hline $\mathrm{C}(1)-\mathrm{N}(1)$ & $1.392(7)$ & $\mathrm{C}(20)-\mathrm{C}(17)-\mathrm{P}(2)$ & $112.9(4)$ \\
\hline $\mathrm{C}(2)-\mathrm{P}(1)$ & $1.806(6)$ & $\mathrm{C}(18)-\mathrm{C}(17)-\mathrm{P}(2)$ & $104.7(4)$ \\
\hline$C(3)-C(4)$ & $1.517(10)$ & $\mathrm{N}(2)-\mathrm{C}(21)-\mathrm{C}(22)$ & $121.4(5)$ \\
\hline$C(3)-C(6)$ & $1.526(11)$ & $\mathrm{C}(21)-\mathrm{C}(22)-\mathrm{P}(3)$ & $110.3(4)$ \\
\hline$C(3)-C(5)$ & $1.547(9)$ & $C(26)-C(23)-C(25)$ & $109.7(5)$ \\
\hline $\mathrm{C}(3)-\mathrm{P}(1)$ & $1.852(6)$ & $C(26)-C(23)-C(24)$ & $109.0(5)$ \\
\hline $\mathrm{C}(7)-\mathrm{C}(9)$ & $1.527(11)$ & $\mathrm{C}(25)-\mathrm{C}(23)-\mathrm{C}(24)$ & $109.3(5)$ \\
\hline$C(7)-C(10)$ & $1.531(9)$ & $\mathrm{C}(26)-\mathrm{C}(23)-\mathrm{P}(3)$ & $110.7(4)$ \\
\hline$C(7)-C(8)$ & $1.533(11)$ & $\mathrm{C}(25)-\mathrm{C}(23)-\mathrm{P}(3)$ & $113.1(4)$ \\
\hline$C(7)-P(1)$ & $1.864(6)$ & $\mathrm{C}(24)-\mathrm{C}(23)-\mathrm{P}(3)$ & $104.9(4)$ \\
\hline $\mathrm{C}(11)-\mathrm{N}(1)$ & $1.319(7)$ & $\mathrm{C}(30)-\mathrm{C}(27)-\mathrm{C}(29)$ & $109.4(5)$ \\
\hline$C(11)-C(12)$ & $1.429(8)$ & $\mathrm{C}(30)-\mathrm{C}(27)-\mathrm{C}(28)$ & $108.8(5)$ \\
\hline $\mathrm{C}(12)-\mathrm{P}(2)$ & $1.841(6)$ & $\mathrm{C}(29)-\mathrm{C}(27)-\mathrm{C}(28)$ & $108.7(5)$ \\
\hline$C(13)-C(16)$ & $1.531(8)$ & $\mathrm{C}(30)-\mathrm{C}(27)-\mathrm{P}(3)$ & $111.0(4)$ \\
\hline$C(13)-C(15)$ & $1.539(8)$ & $\mathrm{C}(29)-\mathrm{C}(27)-\mathrm{P}(3)$ & $113.8(4)$ \\
\hline$C(13)-C(14)$ & $1.539(7)$ & $\mathrm{C}(28)-\mathrm{C}(27)-\mathrm{P}(3)$ & $104.9(4)$ \\
\hline $\mathrm{C}(13)-\mathrm{P}(2)$ & $1.851(6)$ & $\mathrm{C}(32)-\mathrm{C}(31)-\mathrm{N}(2)$ & $120.3(5)$ \\
\hline C(17)-C(19) & $1.529(9)$ & $\mathrm{C}(31)-\mathrm{C}(32)-\mathrm{P}(4)$ & $114.8(5)$ \\
\hline$C(17)-C(20)$ & $1.537(8)$ & $\mathrm{C}(34)-\mathrm{C}(33)-\mathrm{C}(36)$ & $110.5(5)$ \\
\hline$C(17)-C(18)$ & $1.546(8)$ & $\mathrm{C}(34)-\mathrm{C}(33)-\mathrm{C}(35)$ & $108.8(5)$ \\
\hline $\mathrm{C}(17)-\mathrm{P}(2)$ & $1.855(6)$ & $\mathrm{C}(36)-\mathrm{C}(33)-\mathrm{C}(35)$ & $108.7(6)$ \\
\hline $\mathrm{C}(21)-\mathrm{N}(2)$ & $1.312(7)$ & $\mathrm{C}(34)-\mathrm{C}(33)-\mathrm{P}(4)$ & $112.4(4)$ \\
\hline $\mathrm{C}(21)-\mathrm{C}(22)$ & $1.437(8)$ & $\mathrm{C}(36)-\mathrm{C}(33)-\mathrm{P}(4)$ & $110.8(4)$ \\
\hline
\end{tabular}




\begin{tabular}{|c|c|}
\hline C(22)-P(3) & $1.832(6)$ \\
\hline $\mathrm{C}(23)-\mathrm{C}(26)$ & $1.534(9)$ \\
\hline$C(23)-C(25)$ & $1.535(8)$ \\
\hline$C(23)-C(24)$ & $1.544(9)$ \\
\hline $\mathrm{C}(23)-\mathrm{P}(3)$ & $1.853(6)$ \\
\hline $\mathrm{C}(27)-\mathrm{C}(30)$ & $1.520(8)$ \\
\hline C(27)-C(29) & $1.542(8)$ \\
\hline $\mathrm{C}(27)-\mathrm{C}(28)$ & $1.544(8)$ \\
\hline $\mathrm{C}(27)-\mathrm{P}(3)$ & $1.852(5)$ \\
\hline $\mathrm{C}(31)-\mathrm{C}(32)$ & $1.350(8)$ \\
\hline $\mathrm{C}(31)-\mathrm{N}(2)$ & $1.392(7)$ \\
\hline $\mathrm{C}(32)-\mathrm{P}(4)$ & $1.803(6)$ \\
\hline$C(33)-C(34)$ & $1.525(8)$ \\
\hline$C(33)-C(36)$ & $1.526(9)$ \\
\hline C(33)-C(35) & $1.528(9)$ \\
\hline $\mathrm{C}(33)-\mathrm{P}(4)$ & $1.872(6)$ \\
\hline C(37)-C(40) & $1.528(11)$ \\
\hline C(37)-C(39) & $1.529(10)$ \\
\hline $\mathrm{C}(37)-\mathrm{C}(38)$ & $1.546(9)$ \\
\hline $\mathrm{C}(37)-\mathrm{P}(4)$ & $1.857(6)$ \\
\hline $\mathrm{B}(1)-\mathrm{F}(3)$ & $1.371(8)$ \\
\hline $\mathrm{B}(1)-\mathrm{F}(1)$ & $1.386(8)$ \\
\hline $\mathrm{B}(1)-\mathrm{F}(4)$ & $1.392(8)$ \\
\hline $\mathrm{B}(1)-\mathrm{F}(2)$ & $1.406(8)$ \\
\hline $\mathrm{B}(2)-\mathrm{F}(8)$ & $1.373(8)$ \\
\hline $\mathrm{B}(2)-\mathrm{F}(7)$ & $1.385(8)$ \\
\hline $\mathrm{B}(2)-\mathrm{F}(5)$ & $1.389(8)$ \\
\hline $\mathrm{B}(2)-\mathrm{F}(6)$ & $1.406(8)$ \\
\hline $\mathrm{N}(1)-\mathrm{Co}(1)$ & $1.941(4)$ \\
\hline $\mathrm{N}(2)-\mathrm{Co}(2)$ & $1.928(4)$ \\
\hline $\mathrm{P}(1)-\mathrm{Co}(1)$ & $2.2478(15)$ \\
\hline $\mathrm{P}(2)-\mathrm{Co}(1)$ & $2.2493(15)$ \\
\hline $\mathrm{P}(3)-\mathrm{Co}(2)$ & $2.2550(15)$ \\
\hline $\mathrm{P}(4)-\mathrm{Co}(2)$ & $2.2519(15)$ \\
\hline $\mathrm{Cl}(1)-\mathrm{Co}(1)$ & $2.2025(15)$ \\
\hline $\mathrm{Cl}(2)-\mathrm{Co}(2)$ & $2.2013(15)$ \\
\hline $\mathrm{C}(2)-\mathrm{C}(1)-\mathrm{N}(1)$ & $120.0(5)$ \\
\hline $\mathrm{C}(1)-\mathrm{C}(2)-\mathrm{P}(1)$ & $115.3(5)$ \\
\hline$C(4)-C(3)-C(6)$ & $110.6(6)$ \\
\hline
\end{tabular}

\begin{tabular}{|c|c|}
\hline $\mathrm{C}(35)-\mathrm{C}(33)-\mathrm{P}(4)$ & $105.5(4)$ \\
\hline $\mathrm{C}(40)-\mathrm{C}(37)-\mathrm{C}(39)$ & $110.5(6)$ \\
\hline $\mathrm{C}(40)-\mathrm{C}(37)-\mathrm{C}(38)$ & 109.1(6) \\
\hline $\mathrm{C}(39)-\mathrm{C}(37)-\mathrm{C}(38)$ & $108.5(6)$ \\
\hline $\mathrm{C}(40)-\mathrm{C}(37)-\mathrm{P}(4)$ & $110.9(5)$ \\
\hline $\mathrm{C}(39)-\mathrm{C}(37)-\mathrm{P}(4)$ & $105.0(4)$ \\
\hline $\mathrm{C}(38)-\mathrm{C}(37)-\mathrm{P}(4)$ & $112.8(5)$ \\
\hline $\mathrm{F}(3)-\mathrm{B}(1)-\mathrm{F}(1)$ & $110.1(5)$ \\
\hline $\mathrm{F}(3)-\mathrm{B}(1)-\mathrm{F}(4)$ & $111.3(5)$ \\
\hline $\mathrm{F}(1)-\mathrm{B}(1)-\mathrm{F}(4)$ & $110.1(5)$ \\
\hline $\mathrm{F}(3)-\mathrm{B}(1)-\mathrm{F}(2)$ & $109.1(5)$ \\
\hline $\mathrm{F}(1)-\mathrm{B}(1)-\mathrm{F}(2)$ & $108.6(5)$ \\
\hline $\mathrm{F}(4)-\mathrm{B}(1)-\mathrm{F}(2)$ & $107.5(5)$ \\
\hline $\mathrm{F}(8)-\mathrm{B}(2)-\mathrm{F}(7)$ & $110.5(5)$ \\
\hline $\mathrm{F}(8)-\mathrm{B}(2)-\mathrm{F}(5)$ & $110.2(6)$ \\
\hline $\mathrm{F}(7)-\mathrm{B}(2)-\mathrm{F}(5)$ & $109.9(5)$ \\
\hline $\mathrm{F}(8)-\mathrm{B}(2)-\mathrm{F}(6)$ & $109.1(5)$ \\
\hline$F(7)-B(2)-F(6)$ & $109.1(5)$ \\
\hline $\mathrm{F}(5)-\mathrm{B}(2)-\mathrm{F}(6)$ & $107.9(5)$ \\
\hline $\mathrm{C}(11)-\mathrm{N}(1)-\mathrm{C}(1)$ & $117.0(5)$ \\
\hline $\mathrm{C}(11)-\mathrm{N}(1)-\mathrm{Co}(1)$ & $122.2(4)$ \\
\hline $\mathrm{C}(1)-\mathrm{N}(1)-\mathrm{Co}(1)$ & $120.8(4)$ \\
\hline $\mathrm{C}(21)-\mathrm{N}(2)-\mathrm{C}(31)$ & $116.6(5)$ \\
\hline $\mathrm{C}(21)-\mathrm{N}(2)-\mathrm{Co}(2)$ & $122.8(4)$ \\
\hline $\mathrm{C}(31)-\mathrm{N}(2)-\mathrm{Co}(2)$ & $120.6(4)$ \\
\hline $\mathrm{C}(2)-\mathrm{P}(1)-\mathrm{C}(3)$ & $104.4(3)$ \\
\hline $\mathrm{C}(2)-\mathrm{P}(1)-\mathrm{C}(7)$ & $102.5(3)$ \\
\hline$C(3)-P(1)-C(7)$ & $115.9(3)$ \\
\hline $\mathrm{C}(2)-\mathrm{P}(1)-\mathrm{Co}(1)$ & $99.0(2)$ \\
\hline $\mathrm{C}(3)-\mathrm{P}(1)-\mathrm{Co}(1)$ & $115.6(2)$ \\
\hline $\mathrm{C}(7)-\mathrm{P}(1)-\mathrm{Co}(1)$ & $116.0(2)$ \\
\hline $\mathrm{C}(12)-\mathrm{P}(2)-\mathrm{C}(13)$ & $104.0(3)$ \\
\hline $\mathrm{C}(12)-\mathrm{P}(2)-\mathrm{C}(17)$ & $104.9(3)$ \\
\hline $\mathrm{C}(13)-\mathrm{P}(2)-\mathrm{C}(17)$ & $115.0(3)$ \\
\hline $\mathrm{C}(12)-\mathrm{P}(2)-\mathrm{Co}(1)$ & $99.3(2)$ \\
\hline $\mathrm{C}(13)-\mathrm{P}(2)-\mathrm{Co}(1)$ & $116.77(18)$ \\
\hline $\mathrm{C}(17)-\mathrm{P}(2)-\mathrm{Co}(1)$ & $114.11(18)$ \\
\hline $\mathrm{C}(22)-\mathrm{P}(3)-\mathrm{C}(27)$ & $103.7(3)$ \\
\hline $\mathrm{C}(22)-\mathrm{P}(3)-\mathrm{C}(23)$ & $104.6(3)$ \\
\hline
\end{tabular}




$\begin{array}{ll}\mathrm{C}(4)-\mathrm{C}(3)-\mathrm{C}(5) & 108.1(6) \\ \mathrm{C}(6)-\mathrm{C}(3)-\mathrm{C}(5) & 109.2(6) \\ \mathrm{C}(4)-\mathrm{C}(3)-\mathrm{P}(1) & 104.9(4) \\ \mathrm{C}(6)-\mathrm{C}(3)-\mathrm{P}(1) & 111.0(5) \\ \mathrm{C}(5)-\mathrm{C}(3)-\mathrm{P}(1) & 112.9(5) \\ \mathrm{C}(9)-\mathrm{C}(7)-\mathrm{C}(10) & 110.7(6) \\ \mathrm{C}(9)-\mathrm{C}(7)-\mathrm{C}(8) & 109.7(7) \\ \mathrm{C}(10)-\mathrm{C}(7)-\mathrm{C}(8) & 108.0(6) \\ \mathrm{C}(9)-\mathrm{C}(7)-\mathrm{P}(1) & 110.7(5) \\ \mathrm{C}(10)-\mathrm{C}(7)-\mathrm{P}(1) & 112.2(5) \\ \mathrm{C}(8)-\mathrm{C}(7)-\mathrm{P}(1) & 105.5(5) \\ \mathrm{N}(1)-\mathrm{C}(11)-\mathrm{C}(12) & 121.3(5) \\ \mathrm{C}(11)-\mathrm{C}(12)-\mathrm{P}(2) & 110.7(4) \\ \mathrm{C}(16)-\mathrm{C}(13)-\mathrm{C}(15) & 108.3(5) \\ \mathrm{C}(16)-\mathrm{C}(13)-\mathrm{C}(14) & 109.1(5) \\ \mathrm{C}(15)-\mathrm{C}(13)-\mathrm{C}(14) & 108.8(5) \\ \mathrm{C}(16)-\mathrm{C}(13)-\mathrm{P}(2) & 111.5(4) \\ \mathrm{C}(15)-\mathrm{C}(13)-\mathrm{P}(2) & 105.4(4) \\ \mathrm{C}(14)-\mathrm{C}(13)-\mathrm{P}(2) & 113.6(4) \\ \mathrm{C}(19)-\mathrm{C}(17)-\mathrm{C}(20) & 109.9(5) \\ \mathrm{C}(19)-\mathrm{C}(17)-\mathrm{C}(18) & 109.4(5) \\ \mathrm{C}(20)-\mathrm{C}(17)-\mathrm{C}(18) & 109.2(5) \\ & \end{array}$

$\begin{array}{lc}\mathrm{C}(27)-\mathrm{P}(3)-\mathrm{C}(23) & 115.1(3) \\ \mathrm{C}(22)-\mathrm{P}(3)-\mathrm{Co}(2) & 99.5(2) \\ \mathrm{C}(27)-\mathrm{P}(3)-\mathrm{Co}(2) & 116.22(18) \\ \mathrm{C}(23)-\mathrm{P}(3)-\mathrm{Co}(2) & 114.77(19) \\ \mathrm{C}(32)-\mathrm{P}(4)-\mathrm{C}(37) & 104.6(3) \\ \mathrm{C}(32)-\mathrm{P}(4)-\mathrm{C}(33) & 102.6(3) \\ \mathrm{C}(37)-\mathrm{P}(4)-\mathrm{C}(33) & 115.4(3) \\ \mathrm{C}(32)-\mathrm{P}(4)-\mathrm{Co}(2) & 98.9(2) \\ \mathrm{C}(37)-\mathrm{P}(4)-\mathrm{Co}(2) & 115.9(2) \\ \mathrm{C}(33)-\mathrm{P}(4)-\mathrm{Co}(2) & 116.03(19) \\ \mathrm{N}(1)-\mathrm{Co}(1)-\mathrm{Cl}(1) & 178.63(14) \\ \mathrm{N}(1)-\mathrm{Co}(1)-\mathrm{P}(1) & 85.00(14) \\ \mathrm{Cl}(1)-\mathrm{Co}(1)-\mathrm{P}(1) & 94.73(6) \\ \mathrm{N}(1)-\mathrm{Co}(1)-\mathrm{P}(2) & 85.19(14) \\ \mathrm{Cl}(1)-\mathrm{Co}(1)-\mathrm{P}(2) & 95.09(6) \\ \mathrm{P}(1)-\mathrm{Co}(1)-\mathrm{P}(2) & 170.16(6) \\ \mathrm{N}(2)-\mathrm{Co}(2)-\mathrm{Cl}(2) & 178.41(14) \\ \mathrm{N}(2)-\mathrm{Co}(2)-\mathrm{P}(4) & 85.36(14) \\ \mathrm{Cl}(2)-\mathrm{Co}(2)-\mathrm{P}(4) & 94.68(6) \\ \mathrm{N}(2)-\mathrm{Co}(2)-\mathrm{P}(3) & 84.91(13) \\ \mathrm{Cl}(2)-\mathrm{Co}(2)-\mathrm{P}(3) & 95.07(6) \\ \mathrm{P}(4)-\mathrm{Co}(2)-\mathrm{P}(3) & \\ & 170.24(6) \\ & \end{array}$

Table S21. Torsion angles $\left[^{\circ}\right]$ for $\mathbf{8 c}$.

$\begin{array}{lccc}\mathrm{N}(1)-\mathrm{C}(1)-\mathrm{C}(2)-\mathrm{P}(1) & 2.1(8) & \mathrm{C}(19)-\mathrm{C}(17)-\mathrm{P}(2)-\mathrm{C}(13) & -73.6(5) \\ \mathrm{N}(1)-\mathrm{C}(11)-\mathrm{C}(12)-\mathrm{P}(2) & -12.4(8) & \mathrm{C}(20)-\mathrm{C}(17)-\mathrm{P}(2)-\mathrm{C}(13) & 50.0(5) \\ \mathrm{N}(2)-\mathrm{C}(21)-\mathrm{C}(22)-\mathrm{P}(3) & -11.1(8) & \mathrm{C}(18)-\mathrm{C}(17)-\mathrm{P}(2)-\mathrm{C}(13) & 168.7(4) \\ \mathrm{N}(2)-\mathrm{C}(31)-\mathrm{C}(32)-\mathrm{P}(4) & 2.2(7) & \mathrm{C}(19)-\mathrm{C}(17)-\mathrm{P}(2)-\mathrm{Co}(1) & 65.3(5) \\ \mathrm{C}(12)-\mathrm{C}(11)-\mathrm{N}(1)-\mathrm{C}(1) & -172.1(6) & \mathrm{C}(20)-\mathrm{C}(17)-\mathrm{P}(2)-\mathrm{Co}(1) & -171.1(4) \\ \mathrm{C}(12)-\mathrm{C}(11)-\mathrm{N}(1)-\mathrm{Co}(1) & 6.4(8) & \mathrm{C}(18)-\mathrm{C}(17)-\mathrm{P}(2)-\mathrm{Co}(1) & -52.4(4) \\ \mathrm{C}(2)-\mathrm{C}(1)-\mathrm{N}(1)-\mathrm{C}(11) & 177.7(6) & \mathrm{C}(21)-\mathrm{C}(22)-\mathrm{P}(3)-\mathrm{C}(27) & 130.3(5) \\ \mathrm{C}(2)-\mathrm{C}(1)-\mathrm{N}(1)-\mathrm{Co}(1) & -0.8(7) & \mathrm{C}(21)-\mathrm{C}(22)-\mathrm{P}(3)-\mathrm{C}(23) & -108.8(5) \\ \mathrm{C}(22)-\mathrm{C}(21)-\mathrm{N}(2)-\mathrm{C}(31) & -172.7(6) & \mathrm{C}(21)-\mathrm{C}(22)-\mathrm{P}(3)-\mathrm{Co}(2) & 10.1(5) \\ \mathrm{C}(22)-\mathrm{C}(21)-\mathrm{N}(2)-\mathrm{Co}(2) & 5.7(8) & \mathrm{C}(30)-\mathrm{C}(27)-\mathrm{P}(3)-\mathrm{C}(22) & 161.3(4) \\ \mathrm{C}(32)-\mathrm{C}(31)-\mathrm{N}(2)-\mathrm{C}(21) & 177.7(6) & \mathrm{C}(29)-\mathrm{C}(27)-\mathrm{P}(3)-\mathrm{C}(22) & 37.4(5) \\ \mathrm{C}(32)-\mathrm{C}(31)-\mathrm{N}(2)-\mathrm{Co}(2) & -0.7(7) & \mathrm{C}(28)-\mathrm{C}(27)-\mathrm{P}(3)-\mathrm{C}(22) & -81.3(4) \\ \mathrm{C}(1)-\mathrm{C}(2)-\mathrm{P}(1)-\mathrm{C}(3) & -121.6(5) & \mathrm{C}(30)-\mathrm{C}(27)-\mathrm{P}(3)-\mathrm{C}(23) & 47.7(5) \\ \mathrm{C}(1)-\mathrm{C}(2)-\mathrm{P}(1)-\mathrm{C}(7) & 117.3(5) & \mathrm{C}(29)-\mathrm{C}(27)-\mathrm{P}(3)-\mathrm{C}(23) & -76.2(5) \\ \mathrm{C}(1)-\mathrm{C}(2)-\mathrm{P}(1)-\mathrm{C}(1) & -2.1(5) & \mathrm{C}(28)-\mathrm{C}(27)-\mathrm{P}(3)-\mathrm{C}(23) & 165.1(4) \\ & & \mathrm{S} 30 & \end{array}$




\begin{tabular}{|c|c|c|c|}
\hline $\mathrm{C}(4)-\mathrm{C}(3)-\mathrm{P}(1)-\mathrm{C}(2)$ & $77.2(6)$ & $\mathrm{C}(30)-\mathrm{C}(27)-\mathrm{P}(3)-\mathrm{Co}(2)$ & $-90.6(4)$ \\
\hline $\mathrm{C}(6)-\mathrm{C}(3)-\mathrm{P}(1)-\mathrm{C}(2)$ & $-163.2(5)$ & $\mathrm{C}(29)-\mathrm{C}(27)-\mathrm{P}(3)-\mathrm{Co}(2)$ & $145.5(4)$ \\
\hline $\mathrm{C}(5)-\mathrm{C}(3)-\mathrm{P}(1)-\mathrm{C}(2)$ & $-40.3(6)$ & $\mathrm{C}(28)-\mathrm{C}(27)-\mathrm{P}(3)-\mathrm{Co}(2)$ & $26.8(4)$ \\
\hline $\mathrm{C}(4)-\mathrm{C}(3)-\mathrm{P}(1)-\mathrm{C}(7)$ & $-170.9(5)$ & $\mathrm{C}(26)-\mathrm{C}(23)-\mathrm{P}(3)-\mathrm{C}(22)$ & $173.6(4)$ \\
\hline $\mathrm{C}(6)-\mathrm{C}(3)-\mathrm{P}(1)-\mathrm{C}(7)$ & $-51.4(6)$ & $\mathrm{C}(25)-\mathrm{C}(23)-\mathrm{P}(3)-\mathrm{C}(22)$ & $-62.8(5)$ \\
\hline $\mathrm{C}(5)-\mathrm{C}(3)-\mathrm{P}(1)-\mathrm{C}(7)$ & $71.5(6)$ & $\mathrm{C}(24)-\mathrm{C}(23)-\mathrm{P}(3)-\mathrm{C}(22)$ & $56.2(5)$ \\
\hline $\mathrm{C}(4)-\mathrm{C}(3)-\mathrm{P}(1)-\mathrm{Co}(1)$ & $-30.3(6)$ & $\mathrm{C}(26)-\mathrm{C}(23)-\mathrm{P}(3)-\mathrm{C}(27)$ & $-73.2(5)$ \\
\hline $\mathrm{C}(6)-\mathrm{C}(3)-\mathrm{P}(1)-\mathrm{Co}(1)$ & $89.2(5)$ & $\mathrm{C}(25)-\mathrm{C}(23)-\mathrm{P}(3)-\mathrm{C}(27)$ & $50.3(5)$ \\
\hline $\mathrm{C}(5)-\mathrm{C}(3)-\mathrm{P}(1)-\mathrm{Co}(1)$ & $-147.9(5)$ & $\mathrm{C}(24)-\mathrm{C}(23)-\mathrm{P}(3)-\mathrm{C}(27)$ & $169.3(4)$ \\
\hline $\mathrm{C}(9)-\mathrm{C}(7)-\mathrm{P}(1)-\mathrm{C}(2)$ & $-171.7(5)$ & $\mathrm{C}(26)-\mathrm{C}(23)-\mathrm{P}(3)-\mathrm{Co}(2)$ & $65.6(5)$ \\
\hline $\mathrm{C}(10)-\mathrm{C}(7)-\mathrm{P}(1)-\mathrm{C}(2)$ & $64.1(6)$ & $\mathrm{C}(25)-\mathrm{C}(23)-\mathrm{P}(3)-\mathrm{Co}(2)$ & $-170.8(4)$ \\
\hline $\mathrm{C}(8)-\mathrm{C}(7)-\mathrm{P}(1)-\mathrm{C}(2)$ & $-53.2(6)$ & $\mathrm{C}(24)-\mathrm{C}(23)-\mathrm{P}(3)-\mathrm{Co}(2)$ & $-51.8(4)$ \\
\hline $\mathrm{C}(9)-\mathrm{C}(7)-\mathrm{P}(1)-\mathrm{C}(3)$ & $75.3(6)$ & $\mathrm{C}(31)-\mathrm{C}(32)-\mathrm{P}(4)-\mathrm{C}(37)$ & $-122.1(5)$ \\
\hline $\mathrm{C}(10)-\mathrm{C}(7)-\mathrm{P}(1)-\mathrm{C}(3)$ & $-48.9(7)$ & $\mathrm{C}(31)-\mathrm{C}(32)-\mathrm{P}(4)-\mathrm{C}(33)$ & $117.1(5)$ \\
\hline $\mathrm{C}(8)-\mathrm{C}(7)-\mathrm{P}(1)-\mathrm{C}(3)$ & $-166.2(5)$ & $\mathrm{C}(31)-\mathrm{C}(32)-\mathrm{P}(4)-\mathrm{Co}(2)$ & $-2.3(5)$ \\
\hline $\mathrm{C}(9)-\mathrm{C}(7)-\mathrm{P}(1)-\mathrm{Co}(1)$ & $-65.1(6)$ & $\mathrm{C}(40)-\mathrm{C}(37)-\mathrm{P}(4)-\mathrm{C}(32)$ & $-162.9(5)$ \\
\hline $\mathrm{C}(10)-\mathrm{C}(7)-\mathrm{P}(1)-\mathrm{Co}(1)$ & $170.7(5)$ & $\mathrm{C}(39)-\mathrm{C}(37)-\mathrm{P}(4)-\mathrm{C}(32)$ & $77.7(6)$ \\
\hline $\mathrm{C}(8)-\mathrm{C}(7)-\mathrm{P}(1)-\mathrm{Co}(1)$ & $53.4(6)$ & $\mathrm{C}(38)-\mathrm{C}(37)-\mathrm{P}(4)-\mathrm{C}(32)$ & $-40.3(6)$ \\
\hline $\mathrm{C}(11)-\mathrm{C}(12)-\mathrm{P}(2)-\mathrm{C}(13)$ & $132.1(5)$ & $\mathrm{C}(40)-\mathrm{C}(37)-\mathrm{P}(4)-\mathrm{C}(33)$ & $-51.1(5)$ \\
\hline $\mathrm{C}(11)-\mathrm{C}(12)-\mathrm{P}(2)-\mathrm{C}(17)$ & $-106.8(5)$ & $\mathrm{C}(39)-\mathrm{C}(37)-\mathrm{P}(4)-\mathrm{C}(33)$ & $-170.4(5)$ \\
\hline $\mathrm{C}(11)-\mathrm{C}(12)-\mathrm{P}(2)-\mathrm{Co}(1)$ & $11.3(5)$ & $\mathrm{C}(38)-\mathrm{C}(37)-\mathrm{P}(4)-\mathrm{C}(33)$ & $71.6(6)$ \\
\hline$C(16)-C(13)-P(2)-C(12)$ & $160.6(4)$ & $\mathrm{C}(40)-\mathrm{C}(37)-\mathrm{P}(4)-\mathrm{Co}(2)$ & $89.4(5)$ \\
\hline $\mathrm{C}(15)-\mathrm{C}(13)-\mathrm{P}(2)-\mathrm{C}(12)$ & $-82.1(4)$ & $\mathrm{C}(39)-\mathrm{C}(37)-\mathrm{P}(4)-\mathrm{Co}(2)$ & $-30.0(6)$ \\
\hline $\mathrm{C}(14)-\mathrm{C}(13)-\mathrm{P}(2)-\mathrm{C}(12)$ & $36.9(5)$ & $\mathrm{C}(38)-\mathrm{C}(37)-\mathrm{P}(4)-\mathrm{Co}(2)$ & $-147.9(5)$ \\
\hline $\mathrm{C}(16)-\mathrm{C}(13)-\mathrm{P}(2)-\mathrm{C}(17)$ & $46.5(5)$ & $\mathrm{C}(34)-\mathrm{C}(33)-\mathrm{P}(4)-\mathrm{C}(32)$ & $64.6(5)$ \\
\hline $\mathrm{C}(15)-\mathrm{C}(13)-\mathrm{P}(2)-\mathrm{C}(17)$ & $163.8(4)$ & $\mathrm{C}(36)-\mathrm{C}(33)-\mathrm{P}(4)-\mathrm{C}(32)$ & $-171.2(5)$ \\
\hline$C(14)-C(13)-P(2)-C(17)$ & $-77.2(5)$ & $\mathrm{C}(35)-\mathrm{C}(33)-\mathrm{P}(4)-\mathrm{C}(32)$ & $-53.7(5)$ \\
\hline $\mathrm{C}(16)-\mathrm{C}(13)-\mathrm{P}(2)-\mathrm{Co}(1)$ & $-91.3(4)$ & $\mathrm{C}(34)-\mathrm{C}(33)-\mathrm{P}(4)-\mathrm{C}(37)$ & $-48.5(6)$ \\
\hline $\mathrm{C}(15)-\mathrm{C}(13)-\mathrm{P}(2)-\mathrm{Co}(1)$ & $26.0(4)$ & $\mathrm{C}(36)-\mathrm{C}(33)-\mathrm{P}(4)-\mathrm{C}(37)$ & $75.7(5)$ \\
\hline $\mathrm{C}(14)-\mathrm{C}(13)-\mathrm{P}(2)-\mathrm{Co}(1)$ & $145.0(4)$ & $\mathrm{C}(35)-\mathrm{C}(33)-\mathrm{P}(4)-\mathrm{C}(37)$ & $-166.8(4)$ \\
\hline$C(19)-C(17)-P(2)-C(12)$ & $172.9(4)$ & $\mathrm{C}(34)-\mathrm{C}(33)-\mathrm{P}(4)-\mathrm{Co}(2)$ & 171.1(4) \\
\hline $\mathrm{C}(20)-\mathrm{C}(17)-\mathrm{P}(2)-\mathrm{C}(12)$ & $-63.6(5)$ & $\mathrm{C}(36)-\mathrm{C}(33)-\mathrm{P}(4)-\mathrm{Co}(2)$ & $-64.7(5)$ \\
\hline $\mathrm{C}(18)-\mathrm{C}(17)-\mathrm{P}(2)-\mathrm{C}(12)$ & $55.1(4)$ & $\mathrm{C}(35)-\mathrm{C}(33)-\mathrm{P}(4)-\mathrm{Co}(2)$ & $52.8(5)$ \\
\hline
\end{tabular}


2.8 X-ray Single-Crystal Structure Analysis of 9.

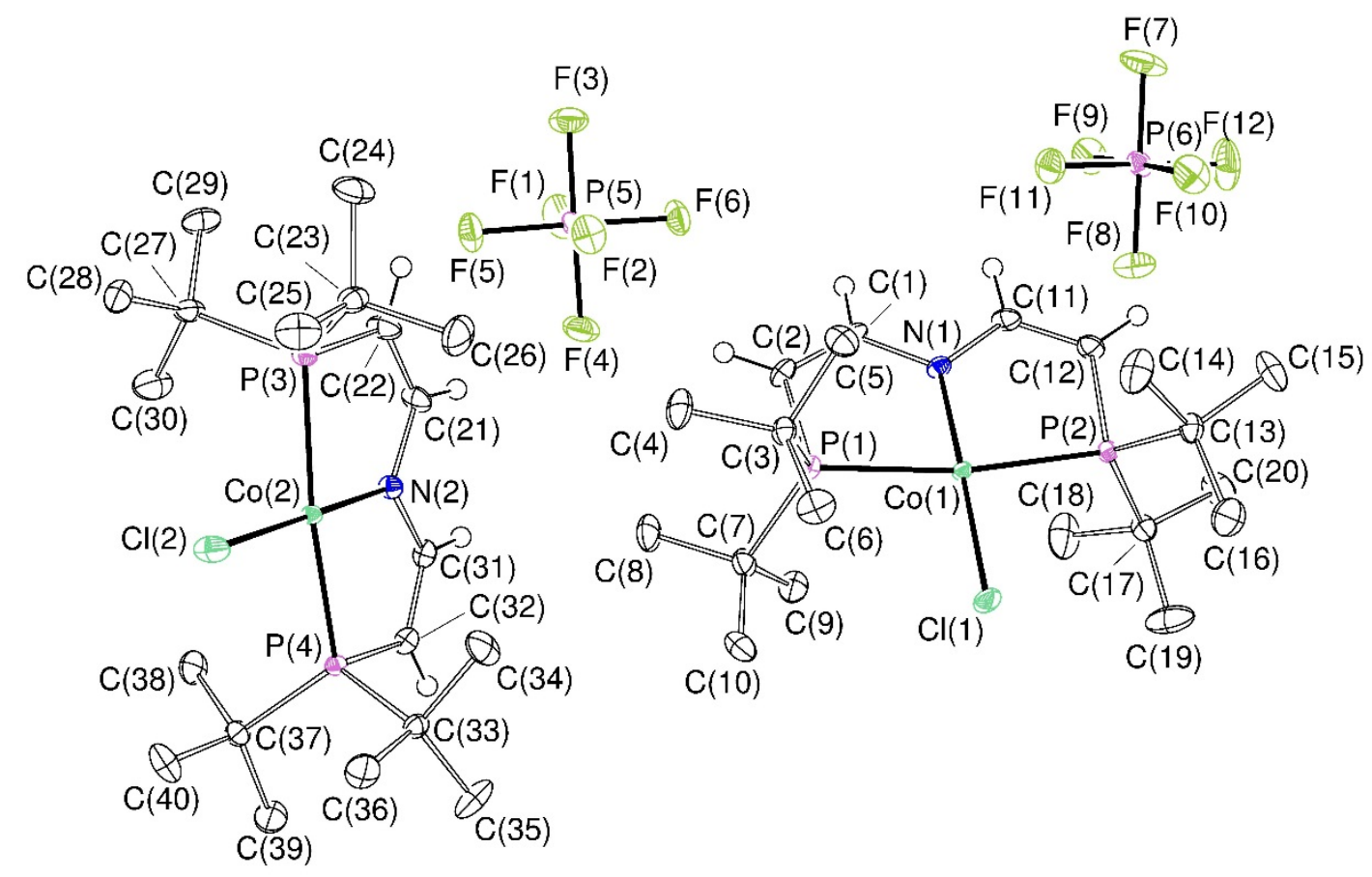

Figure S9. Thermal ellipsoid plot of 9 with the anisotropic displacement parameters drawn at the $50 \%$ probability level. The asymmetric unit contains two complex molecules and two $\mathrm{PF}_{6}$ anions. All hydrogen atoms of the $t$ Bu-groups are omitted for clarity.

Table S22. Crystal data and structure refinement for 9 .

CCDC-No.

Diffractometer and Detector

Identification code

Empirical formula

Formula weight

Temperature

Wavelength

Crystal system

Space group

Unit cell dimensions

Volume

Z

Density (calculated)

Absorption coefficient

$\mathrm{F}(000)$
1452226

Bruker, CMOS

cw_bs_261015_a

$\mathrm{C}_{20} \mathrm{H}_{40} \mathrm{ClCoF}_{6} \mathrm{NP}_{3}$

595.82

100(2) K

$0.71073 \AA$

Monoclinic

$\mathrm{P} 2 / \mathrm{c}$

$\mathrm{a}=19.1704(8) \AA$

$\alpha=90^{\circ}$

$\mathrm{b}=19.1269(8) \AA$

$\beta=94.854(2)^{\circ}$

$\mathrm{c}=14.7424(6) \AA$

$\gamma=90^{\circ}$

$5386.2(4) \AA^{3}$

8

$1.469 \mathrm{Mg} / \mathrm{m}^{3}$

$0.966 \mathrm{~mm}^{-1}$

2480 
Crystal shape and color:

Crystal size

Theta range for data collection

Index ranges

Reflections collected

Independent reflections

Completeness to theta $=25.242^{\circ}$

Absorption correction

Max. and min. transmission

Refinement method

Data / restraints / parameters

Goodness-of-fit on $\mathrm{F}^{2}$

Final $\mathrm{R}$ indices [I $>2$ sigma(I)]

$\mathrm{R}$ indices (all data)

Largest diff. peak and hole
Plate, brown

$0.271 \times 0.224 \times 0.133 \mathrm{~mm}^{3}$

1.985 to $30.599^{\circ}$

$-27<=\mathrm{h}<=27,-27<=\mathrm{k}<=27,-21<=1<=21$

218263

$16532[\mathrm{R}(\mathrm{int})=0.0426]$

$99.9 \%$

Semi-empirical from equivalents

0.7461 and 0.6843

Full-matrix least-squares on $\mathrm{F}^{2}$

$16532 / 0 / 601$

1.111

$\mathrm{R} 1=0.0284$,

$\mathrm{wR} 2=0.0603$

$\mathrm{R} 1=0.0388$,

$w R 2=0.0671$

Table S23. Bond lengths $[\AA]$ and angles $\left[{ }^{\circ}\right]$ for 9.

$\begin{array}{ll}\mathrm{C}(1)-\mathrm{C}(2) & 1.3410(19) \\ \mathrm{C}(1)-\mathrm{N}(1) & 1.3910(16) \\ \mathrm{C}(2)-\mathrm{P}(1) & 1.7967(14) \\ \mathrm{C}(3)-\mathrm{C}(4) & 1.5302(19) \\ \mathrm{C}(3)-\mathrm{C}(5) & 1.5359(19) \\ \mathrm{C}(3)-\mathrm{C}(6) & 1.537(2) \\ \mathrm{C}(3)-\mathrm{P}(1) & 1.8652(13) \\ \mathrm{C}(7)-\mathrm{C}(10) & 1.535(2) \\ \mathrm{C}(7)-\mathrm{C}(8) & 1.537(2) \\ \mathrm{C}(7)-\mathrm{C}(9) & 1.540(2) \\ \mathrm{C}(7)-\mathrm{P}(1) & 1.8622(14) \\ \mathrm{C}(11)-\mathrm{C}(12) & 1.3431(19) \\ \mathrm{C}(11)-\mathrm{N}(1) & 1.3901(17) \\ \mathrm{C}(12)-\mathrm{P}(2) & 1.7982(14) \\ \mathrm{C}(13)-\mathrm{C}(15) & 1.525(2) \\ \mathrm{C}(13)-\mathrm{C}(16) & 1.527(2) \\ \mathrm{C}(13)-\mathrm{C}(14) & 1.534(2) \\ \mathrm{C}(13)-\mathrm{P}(2) & 1.8632(14) \\ \mathrm{C}(17)-\mathrm{C}(20) & 1.5243(19) \\ \mathrm{C}(17)-\mathrm{C}(19) & 1.528(2) \\ \mathrm{C}(17)-\mathrm{C}(18) & 1.534(2) \\ \mathrm{C}(17)-\mathrm{P}(2) & 1.8665(13) \\ & \\ & \end{array}$

$\begin{array}{ll}\mathrm{C}(24)-\mathrm{C}(23)-\mathrm{C}(26) & 108.56(13) \\ \mathrm{C}(25)-\mathrm{C}(23)-\mathrm{P}(3) & 112.03(10) \\ \mathrm{C}(24)-\mathrm{C}(23)-\mathrm{P}(3) & 111.73(10) \\ \mathrm{C}(26)-\mathrm{C}(23)-\mathrm{P}(3) & 105.17(10) \\ \mathrm{C}(29)-\mathrm{C}(27)-\mathrm{C}(28) & 110.02(12) \\ \mathrm{C}(29)-\mathrm{C}(27)-\mathrm{C}(30) & 109.43(13) \\ \mathrm{C}(28)-\mathrm{C}(27)-\mathrm{C}(30) & 109.49(13) \\ \mathrm{C}(29)-\mathrm{C}(27)-\mathrm{P}(3) & 111.26(10) \\ \mathrm{C}(28)-\mathrm{C}(27)-\mathrm{P}(3) & 111.02(10) \\ \mathrm{C}(30)-\mathrm{C}(27)-\mathrm{P}(3) & 105.52(10) \\ \mathrm{C}(32)-\mathrm{C}(31)-\mathrm{N}(2) & 121.40(12) \\ \mathrm{C}(31)-\mathrm{C}(32)-\mathrm{P}(4) & 113.90(10) \\ \mathrm{C}(35)-\mathrm{C}(33)-\mathrm{C}(36) & 110.99(12) \\ \mathrm{C}(35)-\mathrm{C}(33)-\mathrm{C}(34) & 108.85(13) \\ \mathrm{C}(36)-\mathrm{C}(33)-\mathrm{C}(34) & 108.77(12) \\ \mathrm{C}(35)-\mathrm{C}(33)-\mathrm{P}(4) & 111.32(9) \\ \mathrm{C}(36)-\mathrm{C}(33)-\mathrm{P}(4) & 111.82(9) \\ \mathrm{C}(34)-\mathrm{C}(33)-\mathrm{P}(4) & 104.84(9) \\ \mathrm{C}(40)-\mathrm{C}(37)-\mathrm{C}(38) & 108.74(12) \\ \mathrm{C}(40)-\mathrm{C}(37)-\mathrm{C}(39) & 109.96(13) \\ \mathrm{C}(38)-\mathrm{C}(37)-\mathrm{C}(39) & 109.33(13) \\ \mathrm{C}(40)-\mathrm{C}(37)-\mathrm{P}(4) & 112.84(10) \\ & \end{array}$




\begin{tabular}{|c|c|c|c|}
\hline$C(21)-C(22)$ & $1.341(2)$ & $\mathrm{C}(38)-\mathrm{C}(37)-\mathrm{P}(4)$ & $103.91(9)$ \\
\hline $\mathrm{C}(21)-\mathrm{N}(2)$ & $1.3929(17)$ & $\mathrm{C}(39)-\mathrm{C}(37)-\mathrm{P}(4)$ & $111.82(10)$ \\
\hline $\mathrm{C}(22)-\mathrm{P}(3)$ & $1.7906(14)$ & $\mathrm{C}(11)-\mathrm{N}(1)-\mathrm{C}(1)$ & $116.98(11)$ \\
\hline$C(23)-C(25)$ & $1.523(2)$ & $\mathrm{C}(11)-\mathrm{N}(1)-\mathrm{Co}(1)$ & $121.63(9)$ \\
\hline$C(23)-C(24)$ & $1.533(2)$ & $\mathrm{C}(1)-\mathrm{N}(1)-\mathrm{Co}(1)$ & $121.39(9)$ \\
\hline$C(23)-C(26)$ & $1.541(2)$ & $\mathrm{C}(31)-\mathrm{N}(2)-\mathrm{C}(21)$ & $116.63(11)$ \\
\hline $\mathrm{C}(23)-\mathrm{P}(3)$ & $1.8621(14)$ & $\mathrm{C}(31)-\mathrm{N}(2)-\mathrm{Co}(2)$ & $121.50(9)$ \\
\hline$C(27)-C(29)$ & $1.5268(19)$ & $\mathrm{C}(21)-\mathrm{N}(2)-\mathrm{Co}(2)$ & $121.86(9)$ \\
\hline $\mathrm{C}(27)-\mathrm{C}(28)$ & $1.532(2)$ & $\mathrm{C}(2)-\mathrm{P}(1)-\mathrm{C}(7)$ & $105.48(7)$ \\
\hline$C(27)-C(30)$ & $1.532(2)$ & $\mathrm{C}(2)-\mathrm{P}(1)-\mathrm{C}(3)$ & $105.44(6)$ \\
\hline $\mathrm{C}(27)-\mathrm{P}(3)$ & $1.8688(14)$ & $\mathrm{C}(7)-\mathrm{P}(1)-\mathrm{C}(3)$ & $116.21(6)$ \\
\hline$C(31)-C(32)$ & $1.3420(19)$ & $\mathrm{C}(2)-\mathrm{P}(1)-\mathrm{Co}(1)$ & $97.53(5)$ \\
\hline $\mathrm{C}(31)-\mathrm{N}(2)$ & $1.3913(16)$ & $\mathrm{C}(7)-\mathrm{P}(1)-\mathrm{Co}(1)$ & $115.66(5)$ \\
\hline $\mathrm{C}(32)-\mathrm{P}(4)$ & $1.7956(13)$ & $\mathrm{C}(3)-\mathrm{P}(1)-\mathrm{Co}(1)$ & $113.67(4)$ \\
\hline$C(33)-C(35)$ & $1.5286(19)$ & $\mathrm{C}(12)-\mathrm{P}(2)-\mathrm{C}(13)$ & $105.75(6)$ \\
\hline$C(33)-C(36)$ & $1.5293(19)$ & $\mathrm{C}(12)-\mathrm{P}(2)-\mathrm{C}(17)$ & $104.80(6)$ \\
\hline$C(33)-C(34)$ & $1.5369(19)$ & $\mathrm{C}(13)-\mathrm{P}(2)-\mathrm{C}(17)$ & $117.13(6)$ \\
\hline $\mathrm{C}(33)-\mathrm{P}(4)$ & $1.8667(13)$ & $\mathrm{C}(12)-\mathrm{P}(2)-\mathrm{Co}(1)$ & $97.14(5)$ \\
\hline$C(37)-C(40)$ & $1.532(2)$ & $\mathrm{C}(13)-\mathrm{P}(2)-\mathrm{Co}(1)$ & $113.49(4)$ \\
\hline$C(37)-C(38)$ & $1.536(2)$ & $\mathrm{C}(17)-\mathrm{P}(2)-\mathrm{Co}(1)$ & $115.40(4)$ \\
\hline $\mathrm{C}(37)-\mathrm{C}(39)$ & $1.536(2)$ & $\mathrm{C}(22)-\mathrm{P}(3)-\mathrm{C}(23)$ & $105.49(7)$ \\
\hline $\mathrm{C}(37)-\mathrm{P}(4)$ & $1.8643(13)$ & $\mathrm{C}(22)-\mathrm{P}(3)-\mathrm{C}(27)$ & $105.88(7)$ \\
\hline $\mathrm{N}(1)-\mathrm{Co}(1)$ & $1.8518(11)$ & $\mathrm{C}(23)-\mathrm{P}(3)-\mathrm{C}(27)$ & $115.33(6)$ \\
\hline $\mathrm{N}(2)-\mathrm{Co}(2)$ & $1.8552(11)$ & $\mathrm{C}(22)-\mathrm{P}(3)-\mathrm{Co}(2)$ & $97.49(5)$ \\
\hline $\mathrm{F}(1)-\mathrm{P}(5)$ & $1.5903(10)$ & $\mathrm{C}(23)-\mathrm{P}(3)-\mathrm{Co}(2)$ & $119.15(5)$ \\
\hline $\mathrm{F}(2)-\mathrm{P}(5)$ & $1.5978(9)$ & $\mathrm{C}(27)-\mathrm{P}(3)-\mathrm{Co}(2)$ & $110.79(4)$ \\
\hline $\mathrm{F}(3)-\mathrm{P}(5)$ & $1.5922(10)$ & $\mathrm{C}(32)-\mathrm{P}(4)-\mathrm{C}(37)$ & $105.55(6)$ \\
\hline $\mathrm{F}(4)-\mathrm{P}(5)$ & $1.6183(10)$ & $\mathrm{C}(32)-\mathrm{P}(4)-\mathrm{C}(33)$ & $104.31(6)$ \\
\hline $\mathrm{F}(5)-\mathrm{P}(5)$ & $1.5983(9)$ & $\mathrm{C}(37)-\mathrm{P}(4)-\mathrm{C}(33)$ & $116.88(6)$ \\
\hline $\mathrm{F}(6)-\mathrm{P}(5)$ & $1.6127(9)$ & $\mathrm{C}(32)-\mathrm{P}(4)-\mathrm{Co}(2)$ & $97.58(4)$ \\
\hline$F(7)-P(6)$ & $1.5865(11)$ & $\mathrm{C}(37)-\mathrm{P}(4)-\mathrm{Co}(2)$ & $119.16(4)$ \\
\hline $\mathrm{F}(8)-\mathrm{P}(6)$ & $1.6121(10)$ & $\mathrm{C}(33)-\mathrm{P}(4)-\mathrm{Co}(2)$ & $110.12(4)$ \\
\hline $\mathrm{F}(9)-\mathrm{P}(6)$ & $1.5936(10)$ & $\mathrm{F}(1)-\mathrm{P}(5)-\mathrm{F}(3)$ & $90.97(6)$ \\
\hline $\mathrm{F}(10)-\mathrm{P}(6)$ & $1.6007(10)$ & $\mathrm{F}(1)-\mathrm{P}(5)-\mathrm{F}(2)$ & $178.61(6)$ \\
\hline $\mathrm{F}(11)-\mathrm{P}(6)$ & $1.6077(10)$ & $\mathrm{F}(3)-\mathrm{P}(5)-\mathrm{F}(2)$ & $90.31(6)$ \\
\hline $\mathrm{F}(12)-\mathrm{P}(6)$ & $1.5945(11)$ & $\mathrm{F}(1)-\mathrm{P}(5)-\mathrm{F}(5)$ & $90.36(5)$ \\
\hline $\mathrm{P}(1)-\mathrm{Co}(1)$ & $2.2813(4)$ & $\mathrm{F}(3)-\mathrm{P}(5)-\mathrm{F}(5)$ & $90.89(6)$ \\
\hline $\mathrm{P}(2)-\mathrm{Co}(1)$ & $2.2854(4)$ & $F(2)-P(5)-F(5)$ & $90.17(5)$ \\
\hline $\mathrm{P}(3)-\mathrm{Co}(2)$ & $2.2915(4)$ & $F(1)-P(5)-F(6)$ & $89.59(5)$ \\
\hline
\end{tabular}




\begin{tabular}{|c|c|c|c|}
\hline $\mathrm{P}(4)-\mathrm{Co}(2)$ & $2.2904(4)$ & $F(3)-P(5)-F(6)$ & $90.23(5)$ \\
\hline $\mathrm{Cl}(1)-\mathrm{Co}(1)$ & $2.1651(3)$ & $\mathrm{F}(2)-\mathrm{P}(5)-\mathrm{F}(6)$ & $89.85(5)$ \\
\hline $\mathrm{Cl}(2)-\mathrm{Co}(2)$ & $2.1693(4)$ & $\mathrm{F}(5)-\mathrm{P}(5)-\mathrm{F}(6)$ & $178.88(6)$ \\
\hline $\mathrm{C}(2)-\mathrm{C}(1)-\mathrm{N}(1)$ & $121.22(12)$ & $\mathrm{F}(1)-\mathrm{P}(5)-\mathrm{F}(4)$ & $90.01(6)$ \\
\hline $\mathrm{C}(1)-\mathrm{C}(2)-\mathrm{P}(1)$ & $113.87(10)$ & $\mathrm{F}(3)-\mathrm{P}(5)-\mathrm{F}(4)$ & $178.78(6)$ \\
\hline$C(4)-C(3)-C(5)$ & $109.42(12)$ & $\mathrm{F}(2)-\mathrm{P}(5)-\mathrm{F}(4)$ & $88.71(6)$ \\
\hline$C(4)-C(3)-C(6)$ & $110.47(12)$ & $\mathrm{F}(5)-\mathrm{P}(5)-\mathrm{F}(4)$ & $89.83(5)$ \\
\hline$C(5)-C(3)-C(6)$ & $108.37(12)$ & $\mathrm{F}(6)-\mathrm{P}(5)-\mathrm{F}(4)$ & $89.05(5)$ \\
\hline $\mathrm{C}(4)-\mathrm{C}(3)-\mathrm{P}(1)$ & 111.99(10) & $\mathrm{F}(7)-\mathrm{P}(6)-\mathrm{F}(9)$ & $90.83(6)$ \\
\hline $\mathrm{C}(5)-\mathrm{C}(3)-\mathrm{P}(1)$ & $105.27(9)$ & $\mathrm{F}(7)-\mathrm{P}(6)-\mathrm{F}(12)$ & $91.37(7)$ \\
\hline $\mathrm{C}(6)-\mathrm{C}(3)-\mathrm{P}(1)$ & $111.13(9)$ & $\mathrm{F}(9)-\mathrm{P}(6)-\mathrm{F}(12)$ & $90.05(6)$ \\
\hline$C(10)-C(7)-C(8)$ & 109.62(12) & $F(7)-P(6)-F(10)$ & $90.34(6)$ \\
\hline$C(10)-C(7)-C(9)$ & 109.64(12) & $F(9)-P(6)-F(10)$ & $178.64(6)$ \\
\hline $\mathrm{C}(8)-\mathrm{C}(7)-\mathrm{C}(9)$ & 108.96(12) & $F(12)-P(6)-F(10)$ & $90.61(6)$ \\
\hline $\mathrm{C}(10)-\mathrm{C}(7)-\mathrm{P}(1)$ & $112.47(10)$ & $\mathrm{F}(7)-\mathrm{P}(6)-\mathrm{F}(11)$ & $90.11(6)$ \\
\hline $\mathrm{C}(8)-\mathrm{C}(7)-\mathrm{P}(1)$ & $111.42(10)$ & $\mathrm{F}(9)-\mathrm{P}(6)-\mathrm{F}(11)$ & $89.83(6)$ \\
\hline $\mathrm{C}(9)-\mathrm{C}(7)-\mathrm{P}(1)$ & $104.56(9)$ & $\mathrm{F}(12)-\mathrm{P}(6)-\mathrm{F}(11)$ & $178.52(7)$ \\
\hline $\mathrm{C}(12)-\mathrm{C}(11)-\mathrm{N}(1)$ & $120.80(12)$ & $\mathrm{F}(10)-\mathrm{P}(6)-\mathrm{F}(11)$ & $89.48(6)$ \\
\hline $\mathrm{C}(11)-\mathrm{C}(12)-\mathrm{P}(2)$ & $114.40(10)$ & $\mathrm{F}(7)-\mathrm{P}(6)-\mathrm{F}(8)$ & 178.91(7) \\
\hline$C(15)-C(13)-C(16)$ & $109.95(13)$ & $\mathrm{F}(9)-\mathrm{P}(6)-\mathrm{F}(8)$ & $89.64(6)$ \\
\hline$C(15)-C(13)-C(14)$ & $109.49(13)$ & $\mathrm{F}(12)-\mathrm{P}(6)-\mathrm{F}(8)$ & $89.62(7)$ \\
\hline$C(16)-C(13)-C(14)$ & $109.43(13)$ & $\mathrm{F}(10)-\mathrm{P}(6)-\mathrm{F}(8)$ & $89.18(6)$ \\
\hline $\mathrm{C}(15)-\mathrm{C}(13)-\mathrm{P}(2)$ & $111.09(10)$ & $\mathrm{F}(11)-\mathrm{P}(6)-\mathrm{F}(8)$ & $88.91(5)$ \\
\hline $\mathrm{C}(16)-\mathrm{C}(13)-\mathrm{P}(2)$ & $112.17(10)$ & $\mathrm{N}(1)-\mathrm{Co}(1)-\mathrm{Cl}(1)$ & $176.83(4)$ \\
\hline $\mathrm{C}(14)-\mathrm{C}(13)-\mathrm{P}(2)$ & $104.57(9)$ & $\mathrm{N}(1)-\mathrm{Co}(1)-\mathrm{P}(1)$ & $85.60(4)$ \\
\hline$C(20)-C(17)-C(19)$ & $109.69(13)$ & $\mathrm{Cl}(1)-\mathrm{Co}(1)-\mathrm{P}(1)$ & $94.024(14)$ \\
\hline $\mathrm{C}(20)-\mathrm{C}(17)-\mathrm{C}(18)$ & $108.96(13)$ & $\mathrm{N}(1)-\mathrm{Co}(1)-\mathrm{P}(2)$ & $85.77(4)$ \\
\hline $\mathrm{C}(19)-\mathrm{C}(17)-\mathrm{C}(18)$ & $109.23(15)$ & $\mathrm{Cl}(1)-\mathrm{Co}(1)-\mathrm{P}(2)$ & $94.728(13)$ \\
\hline $\mathrm{C}(20)-\mathrm{C}(17)-\mathrm{P}(2)$ & $111.73(10)$ & $\mathrm{P}(1)-\mathrm{Co}(1)-\mathrm{P}(2)$ & $171.053(14)$ \\
\hline C(19)-C(17)-P(2) & $112.09(10)$ & $\mathrm{N}(2)-\mathrm{Co}(2)-\mathrm{Cl}(2)$ & $179.03(4)$ \\
\hline $\mathrm{C}(18)-\mathrm{C}(17)-\mathrm{P}(2)$ & 104.99(10) & $\mathrm{N}(2)-\mathrm{Co}(2)-\mathrm{P}(4)$ & $85.46(4)$ \\
\hline $\mathrm{C}(22)-\mathrm{C}(21)-\mathrm{N}(2)$ & $120.71(12)$ & $\mathrm{Cl}(2)-\mathrm{Co}(2)-\mathrm{P}(4)$ & $94.402(13)$ \\
\hline $\mathrm{C}(21)-\mathrm{C}(22)-\mathrm{P}(3)$ & $114.52(10)$ & $\mathrm{N}(2)-\mathrm{Co}(2)-\mathrm{P}(3)$ & $85.34(4)$ \\
\hline $\mathrm{C}(25)-\mathrm{C}(23)-\mathrm{C}(24)$ & $110.26(13)$ & $\mathrm{Cl}(2)-\mathrm{Co}(2)-\mathrm{P}(3)$ & $94.812(13)$ \\
\hline $\mathrm{C}(25)-\mathrm{C}(23)-\mathrm{C}(26)$ & $108.88(13)$ & $\mathrm{P}(4)-\mathrm{Co}(2)-\mathrm{P}(3)$ & $170.775(14)$ \\
\hline
\end{tabular}

Table S24. Torsion angles $\left[^{\circ}\right]$ for 9.

$\begin{array}{ll}\mathrm{N}(1)-\mathrm{C}(1)-\mathrm{C}(2)-\mathrm{P}(1) & 0.69(18) \\ \mathrm{N}(1)-\mathrm{C}(11)-\mathrm{C}(12)-\mathrm{P}(2) & 2.11(18)\end{array}$




\begin{tabular}{|c|c|c|c|}
\hline $\mathrm{N}(2)-\mathrm{C}(21)-\mathrm{C}(22)-\mathrm{P}(3)$ & $-0.4(2)$ & $\mathrm{C}(21)-\mathrm{C}(22)-\mathrm{P}(3)-\mathrm{C}(23)$ & $125.04(12)$ \\
\hline $\mathrm{N}(2)-\mathrm{C}(31)-\mathrm{C}(32)-\mathrm{P}(4)$ & $-1.57(17)$ & $\mathrm{C}(21)-\mathrm{C}(22)-\mathrm{P}(3)-\mathrm{C}(27)$ & $-112.25(13)$ \\
\hline $\mathrm{C}(12)-\mathrm{C}(11)-\mathrm{N}(1)-\mathrm{C}(1)$ & $-178.21(13)$ & $\mathrm{C}(21)-\mathrm{C}(22)-\mathrm{P}(3)-\mathrm{Co}(2)$ & $1.93(13)$ \\
\hline $\mathrm{C}(12)-\mathrm{C}(11)-\mathrm{N}(1)-\mathrm{Co}(1)$ & $2.31(18)$ & $\mathrm{C}(25)-\mathrm{C}(23)-\mathrm{P}(3)-\mathrm{C}(22)$ & $-170.89(11)$ \\
\hline $\mathrm{C}(2)-\mathrm{C}(1)-\mathrm{N}(1)-\mathrm{C}(11)$ & $-174.73(13)$ & $\mathrm{C}(24)-\mathrm{C}(23)-\mathrm{P}(3)-\mathrm{C}(22)$ & $64.82(12)$ \\
\hline $\mathrm{C}(2)-\mathrm{C}(1)-\mathrm{N}(1)-\mathrm{Co}(1)$ & $4.76(18)$ & $\mathrm{C}(26)-\mathrm{C}(23)-\mathrm{P}(3)-\mathrm{C}(22)$ & $-52.77(12)$ \\
\hline $\mathrm{C}(32)-\mathrm{C}(31)-\mathrm{N}(2)-\mathrm{C}(21)$ & $178.16(13)$ & $\mathrm{C}(25)-\mathrm{C}(23)-\mathrm{P}(3)-\mathrm{C}(27)$ & $72.66(12)$ \\
\hline $\mathrm{C}(32)-\mathrm{C}(31)-\mathrm{N}(2)-\mathrm{Co}(2)$ & $-1.98(17)$ & $\mathrm{C}(24)-\mathrm{C}(23)-\mathrm{P}(3)-\mathrm{C}(27)$ & $-51.63(13)$ \\
\hline $\mathrm{C}(22)-\mathrm{C}(21)-\mathrm{N}(2)-\mathrm{C}(31)$ & $177.81(14)$ & $\mathrm{C}(26)-\mathrm{C}(23)-\mathrm{P}(3)-\mathrm{C}(27)$ & $-169.22(11)$ \\
\hline $\mathrm{C}(22)-\mathrm{C}(21)-\mathrm{N}(2)-\mathrm{Co}(2)$ & $-2.05(19)$ & $\mathrm{C}(25)-\mathrm{C}(23)-\mathrm{P}(3)-\mathrm{Co}(2)$ & $-62.87(11)$ \\
\hline $\mathrm{C}(1)-\mathrm{C}(2)-\mathrm{P}(1)-\mathrm{C}(7)$ & $-123.51(11)$ & $\mathrm{C}(24)-\mathrm{C}(23)-\mathrm{P}(3)-\mathrm{Co}(2)$ & $172.84(9)$ \\
\hline $\mathrm{C}(1)-\mathrm{C}(2)-\mathrm{P}(1)-\mathrm{C}(3)$ & $112.98(11)$ & $\mathrm{C}(26)-\mathrm{C}(23)-\mathrm{P}(3)-\mathrm{Co}(2)$ & $55.26(12)$ \\
\hline $\mathrm{C}(1)-\mathrm{C}(2)-\mathrm{P}(1)-\mathrm{Co}(1)$ & $-4.19(11)$ & $\mathrm{C}(29)-\mathrm{C}(27)-\mathrm{P}(3)-\mathrm{C}(22)$ & $-35.88(12)$ \\
\hline $\mathrm{C}(10)-\mathrm{C}(7)-\mathrm{P}(1)-\mathrm{C}(2)$ & $-165.65(10)$ & $\mathrm{C}(28)-\mathrm{C}(27)-\mathrm{P}(3)-\mathrm{C}(22)$ & $-158.77(10)$ \\
\hline $\mathrm{C}(8)-\mathrm{C}(7)-\mathrm{P}(1)-\mathrm{C}(2)$ & $-42.10(12)$ & $\mathrm{C}(30)-\mathrm{C}(27)-\mathrm{P}(3)-\mathrm{C}(22)$ & $82.70(12)$ \\
\hline $\mathrm{C}(9)-\mathrm{C}(7)-\mathrm{P}(1)-\mathrm{C}(2)$ & $75.45(10)$ & $\mathrm{C}(29)-\mathrm{C}(27)-\mathrm{P}(3)-\mathrm{C}(23)$ & $80.34(12)$ \\
\hline $\mathrm{C}(10)-\mathrm{C}(7)-\mathrm{P}(1)-\mathrm{C}(3)$ & $-49.27(12)$ & $\mathrm{C}(28)-\mathrm{C}(27)-\mathrm{P}(3)-\mathrm{C}(23)$ & $-42.54(12)$ \\
\hline $\mathrm{C}(8)-\mathrm{C}(7)-\mathrm{P}(1)-\mathrm{C}(3)$ & $74.28(12)$ & $\mathrm{C}(30)-\mathrm{C}(27)-\mathrm{P}(3)-\mathrm{C}(23)$ & $-161.07(11)$ \\
\hline $\mathrm{C}(9)-\mathrm{C}(7)-\mathrm{P}(1)-\mathrm{C}(3)$ & $-168.17(9)$ & $\mathrm{C}(29)-\mathrm{C}(27)-\mathrm{P}(3)-\mathrm{Co}(2)$ & $-140.53(9)$ \\
\hline $\mathrm{C}(10)-\mathrm{C}(7)-\mathrm{P}(1)-\mathrm{Co}(1)$ & $87.86(10)$ & $\mathrm{C}(28)-\mathrm{C}(27)-\mathrm{P}(3)-\mathrm{Co}(2)$ & $96.58(10)$ \\
\hline $\mathrm{C}(8)-\mathrm{C}(7)-\mathrm{P}(1)-\mathrm{Co}(1)$ & $-148.59(9)$ & $\mathrm{C}(30)-\mathrm{C}(27)-\mathrm{P}(3)-\mathrm{Co}(2)$ & $-21.95(12)$ \\
\hline $\mathrm{C}(9)-\mathrm{C}(7)-\mathrm{P}(1)-\mathrm{Co}(1)$ & $-31.04(11)$ & $\mathrm{C}(31)-\mathrm{C}(32)-\mathrm{P}(4)-\mathrm{C}(37)$ & $126.52(10)$ \\
\hline $\mathrm{C}(4)-\mathrm{C}(3)-\mathrm{P}(1)-\mathrm{C}(2)$ & $62.10(12)$ & $\mathrm{C}(31)-\mathrm{C}(32)-\mathrm{P}(4)-\mathrm{C}(33)$ & $-109.74(11)$ \\
\hline $\mathrm{C}(5)-\mathrm{C}(3)-\mathrm{P}(1)-\mathrm{C}(2)$ & $-56.71(10)$ & $\mathrm{C}(31)-\mathrm{C}(32)-\mathrm{P}(4)-\mathrm{Co}(2)$ & $3.34(10)$ \\
\hline $\mathrm{C}(6)-\mathrm{C}(3)-\mathrm{P}(1)-\mathrm{C}(2)$ & $-173.82(10)$ & $\mathrm{C}(40)-\mathrm{C}(37)-\mathrm{P}(4)-\mathrm{C}(32)$ & $-179.98(11)$ \\
\hline $\mathrm{C}(4)-\mathrm{C}(3)-\mathrm{P}(1)-\mathrm{C}(7)$ & $-54.29(12)$ & $\mathrm{C}(38)-\mathrm{C}(37)-\mathrm{P}(4)-\mathrm{C}(32)$ & $-62.37(10)$ \\
\hline $\mathrm{C}(5)-\mathrm{C}(3)-\mathrm{P}(1)-\mathrm{C}(7)$ & $-173.10(9)$ & $\mathrm{C}(39)-\mathrm{C}(37)-\mathrm{P}(4)-\mathrm{C}(32)$ & $55.44(12)$ \\
\hline $\mathrm{C}(6)-\mathrm{C}(3)-\mathrm{P}(1)-\mathrm{C}(7)$ & $69.79(11)$ & $\mathrm{C}(40)-\mathrm{C}(37)-\mathrm{P}(4)-\mathrm{C}(33)$ & $64.63(12)$ \\
\hline $\mathrm{C}(4)-\mathrm{C}(3)-\mathrm{P}(1)-\mathrm{Co}(1)$ & $167.74(9)$ & $\mathrm{C}(38)-\mathrm{C}(37)-\mathrm{P}(4)-\mathrm{C}(33)$ & $-177.76(9)$ \\
\hline $\mathrm{C}(5)-\mathrm{C}(3)-\mathrm{P}(1)-\mathrm{Co}(1)$ & $48.93(10)$ & C(39)-C(37)-P(4)-C(33) & $-59.95(13)$ \\
\hline $\mathrm{C}(6)-\mathrm{C}(3)-\mathrm{P}(1)-\mathrm{Co}(1)$ & $-68.18(10)$ & $\mathrm{C}(40)-\mathrm{C}(37)-\mathrm{P}(4)-\mathrm{Co}(2)$ & $-71.80(11)$ \\
\hline $\mathrm{C}(11)-\mathrm{C}(12)-\mathrm{P}(2)-\mathrm{C}(13)$ & $-121.16(11)$ & $\mathrm{C}(38)-\mathrm{C}(37)-\mathrm{P}(4)-\mathrm{Co}(2)$ & $45.81(10)$ \\
\hline $\mathrm{C}(11)-\mathrm{C}(12)-\mathrm{P}(2)-\mathrm{C}(17)$ & $114.47(11)$ & $\mathrm{C}(39)-\mathrm{C}(37)-\mathrm{P}(4)-\mathrm{Co}(2)$ & $163.62(10)$ \\
\hline $\mathrm{C}(11)-\mathrm{C}(12)-\mathrm{P}(2)-\mathrm{Co}(1)$ & $-4.24(11)$ & $\mathrm{C}(35)-\mathrm{C}(33)-\mathrm{P}(4)-\mathrm{C}(32)$ & $-45.24(12)$ \\
\hline $\mathrm{C}(15)-\mathrm{C}(13)-\mathrm{P}(2)-\mathrm{C}(12)$ & $-46.47(12)$ & $\mathrm{C}(36)-\mathrm{C}(33)-\mathrm{P}(4)-\mathrm{C}(32)$ & $-170.03(10)$ \\
\hline $\mathrm{C}(16)-\mathrm{C}(13)-\mathrm{P}(2)-\mathrm{C}(12)$ & $-169.96(11)$ & $\mathrm{C}(34)-\mathrm{C}(33)-\mathrm{P}(4)-\mathrm{C}(32)$ & $72.29(11)$ \\
\hline $\mathrm{C}(14)-\mathrm{C}(13)-\mathrm{P}(2)-\mathrm{C}(12)$ & $71.54(11)$ & $\mathrm{C}(35)-\mathrm{C}(33)-\mathrm{P}(4)-\mathrm{C}(37)$ & $70.83(12)$ \\
\hline $\mathrm{C}(15)-\mathrm{C}(13)-\mathrm{P}(2)-\mathrm{C}(17)$ & $69.81(12)$ & $\mathrm{C}(36)-\mathrm{C}(33)-\mathrm{P}(4)-\mathrm{C}(37)$ & $-53.96(12)$ \\
\hline$C(16)-C(13)-P(2)-C(17)$ & $-53.68(12)$ & $\mathrm{C}(34)-\mathrm{C}(33)-\mathrm{P}(4)-\mathrm{C}(37)$ & $-171.64(10)$ \\
\hline
\end{tabular}




$\begin{array}{llll}\mathrm{C}(14)-\mathrm{C}(13)-\mathrm{P}(2)-\mathrm{C}(17) & -172.17(10) & \mathrm{C}(35)-\mathrm{C}(33)-\mathrm{P}(4)-\mathrm{Co}(2) & -149.04(10) \\ \mathrm{C}(15)-\mathrm{C}(13)-\mathrm{P}(2)-\mathrm{Co}(1) & -151.74(9) & \mathrm{C}(36)-\mathrm{C}(33)-\mathrm{P}(4)-\mathrm{Co}(2) & 86.17(10) \\ \mathrm{C}(16)-\mathrm{C}(13)-\mathrm{P}(2)-\mathrm{Co}(1) & 84.76(11) & \mathrm{C}(34)-\mathrm{C}(33)-\mathrm{P}(4)-\mathrm{Co}(2) & -31.51(10) \\ \mathrm{C}(14)-\mathrm{C}(13)-\mathrm{P}(2)-\mathrm{Co}(1) & -33.73(11) & \mathrm{C}(11)-\mathrm{N}(1)-\mathrm{Co}(1)-\mathrm{P}(1) & 173.48(10) \\ \mathrm{C}(20)-\mathrm{C}(17)-\mathrm{P}(2)-\mathrm{C}(12) & 51.28(12) & \mathrm{C}(1)-\mathrm{N}(1)-\mathrm{Co}(1)-\mathrm{P}(1) & -5.98(10) \\ \mathrm{C}(19)-\mathrm{C}(17)-\mathrm{P}(2)-\mathrm{C}(12) & 174.86(12) & \mathrm{C}(11)-\mathrm{N}(1)-\mathrm{Co}(1)-\mathrm{P}(2) & -4.18(10) \\ \mathrm{C}(18)-\mathrm{C}(17)-\mathrm{P}(2)-\mathrm{C}(12) & -66.68(12) & \mathrm{C}(1)-\mathrm{N}(1)-\mathrm{Co}(1)-\mathrm{P}(2) & 176.36(10) \\ \mathrm{C}(20)-\mathrm{C}(17)-\mathrm{P}(2)-\mathrm{C}(13) & -65.52(13) & \mathrm{C}(31)-\mathrm{N}(2)-\mathrm{Co}(2)-\mathrm{P}(4) & 3.40(10) \\ \mathrm{C}(19)-\mathrm{C}(17)-\mathrm{P}(2)-\mathrm{C}(13) & 58.06(13) & \mathrm{C}(21)-\mathrm{N}(2)-\mathrm{Co}(2)-\mathrm{P}(4) & -176.74(11) \\ \mathrm{C}(18)-\mathrm{C}(17)-\mathrm{P}(2)-\mathrm{C}(13) & 176.52(11) & \mathrm{C}(31)-\mathrm{N}(2)-\mathrm{Co}(2)-\mathrm{P}(3) & -177.21(10) \\ \mathrm{C}(20)-\mathrm{C}(17)-\mathrm{P}(2)-\mathrm{Co}(1) & 156.82(10) & \mathrm{C}(21)-\mathrm{N}(2)-\mathrm{Co}(2)-\mathrm{P}(3) & 2.64(10)\end{array}$

